\title{
Adaptive IGAFEM with optimal convergence rates: Hierarchical B-splines
}

\author{
Gregor Gantner*, Daniel Haberlik ${ }^{\dagger}$ and Dirk Praetorius ${ }^{\ddagger}$ \\ Institute for Analysis and Scientific Computing, TU Wien, \\ Wiedner Hauptstraße 8-10, A-1040 Wien, Austria \\ *Gregor.Gantner@asc.tuwien.ac.at \\ ${ }^{\dagger}$ Daniel.Haberlik@asc.tuwien.ac.at \\ ${ }^{\ddagger}$ Dirk.Praetorius@asc.tuwien.ac.at
}

Received 9 February 2017

Revised 21 August 2017

Accepted 2 September 2017

Published 2 November 2017

Communicated by F. Brezzi

\begin{abstract}
We consider an adaptive algorithm for finite element methods for the isogeometric analysis (IGAFEM) of elliptic (possibly non-symmetric) second-order partial differential equations in arbitrary space dimension $d \geq 2$. We employ hierarchical B-splines of arbitrary degree and different order of smoothness. We propose a refinement strategy to generate a sequence of locally refined meshes and corresponding discrete solutions. Adaptivity is driven by some weighted residual a posteriori error estimator. We prove linear convergence of the error estimator (respectively, the sum of energy error plus data oscillations) with optimal algebraic rates. Numerical experiments underpin the theoretical findings.
\end{abstract}

Keywords: Isogeometric analysis; hierarchical splines; adaptivity.

AMS Subject Classification: 41A15, 65D07, 65N12, 65N30

\section{Introduction}

\subsection{Adaptivity in isogeometric analysis}

The central idea of isogeometric analysis (IGA) $29|16| 3$ is to use the same ansatz functions for the discretization of the partial differential equation (PDE) as for the representation of the problem geometry in computer-aided design (CAD). The CAD standard for spline representation in a multivariate setting relies on tensor-product B-splines. However, to allow for adaptive refinement, several extensions of the B-spline model have recently emerged, e.g. analysis-suitable T-splines, $\stackrel{40 \mid 5}{4}$ hierarchical splines, $\underline{45|26| 32}$ or LR-splines $\frac{18 \mid 30}{}$; see also Refs. 31 and 28 for a comparison of

This is an open access article published by World Scientific Publishing and distributed under the terms of the Creative Commons Attribution (CC BY) 4.0 License, which permits use, distribution and reproduction in any medium, provided the original author(s) and source are credited. 
these approaches. All these concepts have been studied via numerical experiments. For standard FEM with globally continuous piecewise polynomials, adaptivity is well understood; see, e.g. Refs. 19, 35, 8, 42, 15, 21 and 14 for milestones on convergence and optimal convergence rates. In the frame of isogeometric boundary element methods (IGABEM), we also mention our recent work ${ }^{23}$ which shows linear convergence with optimal rates for some adaptive isogeometric boundary element method in $2 \mathrm{D}$ from Refs. 24 and 22 , where it is however sufficient to use the span of univariate non-uniform rational B-splines (NURBS). However, so far there exists only little literature concerning the thorough mathematical analysis of adaptive isogeometric finite element methods (IGAFEM): Ref. 9 investigates an estimator reduction of an IGAFEM with certain hierarchical splines introduced in Ref. 10, Reference 11 investigates linear convergence of an IGAFEM with truncated hierarchical B-splines introduced in Ref. 26. In the continuation of Ref. 11, Ref. 13 studied the corresponding mesh-refinement strategy together with some refinement-related properties for the proof of optimal convergence. At the time our paper was written, the mathematical proof that the adaptive strategy of Ref. 11 leads to optimal convergence rates, was still missing in the literature. While the current paper was under review, optimal convergence behavior for symmetric PDEs has been proved in Ref. 12, but not been underpinned by numerical experiments. The algorithm of Ref. 11 was designed for truncated hierarchical B-splines only and the use of hierarchical Bsplines may lead to non-sparse Galerkin matrices. It is important to note that the procedure of truncation requires a specific construction that entails complicated supports of the basis functions, which are in general not even connected, and their use may produce an overhead with an adaptive strategy that cannot be neglected.

\subsection{Model problem}

On the bounded Lipschitz domain $\Omega \subset \mathbb{R}^{d}$ with $d \geq 2$, we consider a general secondorder linear elliptic PDE in divergence form with homogeneous Dirichlet boundary conditions:

$$
\begin{aligned}
\mathcal{L} u:=-\operatorname{div}(\mathbf{A} \nabla u)+\mathbf{b} \cdot \nabla u+c u & =f & & \text { in } \Omega, \\
u & =0 & & \text { on } \partial \Omega .
\end{aligned}
$$

We pose the following regularity assumptions on the coefficients: $\mathbf{A}(x) \in \mathbb{R}_{\text {sym }}^{d \times d}$ is a symmetric matrix with $\mathbf{A} \in W^{1, \infty}(\Omega)$. The vector $\mathbf{b}(x) \in \mathbb{R}^{d}$ and the scalar $c(x) \in \mathbb{R}$ satisfy $\mathbf{b}, c \in L^{\infty}(\Omega)$. We interpret $\mathcal{L}$ in its weak form and define the corresponding bilinear form

$$
\langle w, v\rangle_{\mathcal{L}}:=\int_{\Omega} \mathbf{A}(x) \nabla w(x) \cdot \nabla v(x)+\mathbf{b}(x) \cdot \nabla w(x) v(x)+c(x) w(x) v(x) d x .
$$

The bilinear form is continuous, i.e. it holds that

$$
\begin{aligned}
\langle w, v\rangle_{\mathcal{L}} \leq & \left(\|\mathbf{A}\|_{L^{\infty}(\Omega)}+\|\mathbf{b}\|_{L^{\infty}(\Omega)}\right. \\
& \left.+\|c\|_{L^{\infty}(\Omega)}\right)\|w\|_{H^{1}(\Omega)}\|v\|_{H^{1}(\Omega)} \text { for all } v, w \in H^{1}(\Omega) .
\end{aligned}
$$


Additionally, we suppose ellipticity of $\langle\cdot, \cdot\rangle_{\mathcal{L}}$ on $H_{0}^{1}(\Omega)$, i.e.

$$
\langle v, v\rangle_{\mathcal{L}} \geq C_{\mathrm{ell}}\|v\|_{H^{1}(\Omega)}^{2} \quad \text { for all } v \in H_{0}^{1}(\Omega) .
$$

Note that 1.4 is for instance satisfied if $\mathbf{A}(x)$ is uniformly positive definite and if $\mathbf{b} \in H(\operatorname{div}, \Omega)$ with $-\frac{1}{2} \operatorname{div} \mathbf{b}(x)+c(x) \geq 0$ almost everywhere in $\Omega$.

Overall, the boundary value problem (1.1) fits into the setting of the LaxMilgram theorem and therefore admits a unique solution $u \in H_{0}^{1}(\Omega)$ to the weak formulation

$$
\langle u, v\rangle_{\mathcal{L}}=\int_{\Omega} f v d x \quad \text { for all } v \in H_{0}^{1}(\Omega) .
$$

Finally, we note that the additional regularity $\mathbf{A} \in W^{1, \infty}(\Omega)$ (instead of $\mathbf{A} \in$ $\left.L^{\infty}(\Omega)\right)$ is only required for the well-posedness of the residual a posteriori error estimator, see Sec. 2 .

\subsection{Outline and contributions}

The remainder of this work is roughly organized as follows. Section 2 provides an abstract framework for adaptive mesh-refinement for conforming FEM for the model problem 1.1. Its main result is Theorem 2.1 which states optimal convergence behavior of some standard adaptive algorithm. Section 3 considers conforming FEM based on hierarchical splines. Its main result is Theorem 3.1 which states that hierarchical splines fit into the framework of Sec. 2. The proofs of Theorem 2.1] and Theorem 3.1] are given in Secs. 4 and 5. respectively. Three numerical experiments in Sec. 6] underpin the optimal convergence behavior of adaptive IGAFEM with hierarchical splines.

In more detail, the contribution of Sec. 2 can be paraphrased as follows: we formulate the standard adaptive strategy (Algorithm 2.1) driven by some residual a posteriori error estimator (2.9) in the frame of conforming FEM. We formulate four assumptions (M1)-(M4) for the underlying meshes (Sec. 2.1), five assumptions (R1)-(R5) on the mesh-refinement (Sec. 2.2), six assumptions (S1)-(S6) on the FEM spaces (Sec. 2.3), and four assumptions (O1)-(O4) on the data approximation spaces (Sec. 2.5). First, these assumptions are sufficient to guarantee that the error estimator $\eta_{\bullet}$ associated with the FEM solution $U_{\bullet} \in \mathcal{X}_{\bullet} \subset H_{0}^{1}(\Omega)$ is efficient and reliable, i.e. there exist $C_{\text {eff }}, C_{\text {rel }}>0$ such that

$$
\begin{aligned}
C_{\mathrm{eff}^{-1}} \eta_{\bullet} & \leq \inf _{V_{\bullet} \in \mathcal{X}_{\bullet}}\left(\left\|u-V_{\bullet}\right\|_{H^{1}(\Omega)}+\operatorname{osc}_{\bullet}\left(V_{\bullet}\right)\right) \\
& \leq\left\|u-U_{\bullet}\right\|_{H^{1}(\Omega)}+\operatorname{osc}_{\bullet}\left(U_{\bullet}\right) \leq C_{\mathrm{rel}} \eta_{\bullet},
\end{aligned}
$$

where $\operatorname{osc}_{\bullet}(\cdot)$ denotes certain data oscillation terms. Second, Theorem 2.1 states that Algorithm 2.1 leads to linear convergence with optimal rates in the spirit of Refs. 15 and 14 let $\eta_{\ell}$ denote the error estimator in the $\ell$ th step of the adaptive algorithm. Then, there exist $C>0$ and $0<q<1$ such that

$$
\eta_{\ell+n} \leq C q^{n} \eta_{\ell} \quad \text { for all } \ell, n \in \mathbb{N}_{0}
$$


Moreover, for sufficiently small marking parameters in Algorithm 2.1, the estimator (respectively, the so-called total error $\left\|u-U_{\ell}\right\|_{H^{1}(\Omega)}+\operatorname{osc}_{\ell}\left(U_{\ell}\right)$; see (1.6)) decays even with the optimal algebraic convergence rate in the sense of certain nonlinear approximation classes (Sec. 2.7).

In explicit terms, we identify sufficient conditions of the underlying meshes, the local FEM spaces, as well as the employed (local) mesh-refinement rule which guarantee that the related residual a posteriori error estimator satisfies the axioms of adaptivity from Ref. 14 so that linear convergence with optimal rates for the standard adaptive algorithm follows. While we exploit this framework only for IGAFEM with hierarchical splines, it might possibly serve as promising starting point to analyze different technologies for adaptive IGAFEM like (analysis-suitable) T-splines or LR-splines, as well as for other conforming discretizations like the virtual element method (VEM)! ${ }^{4}$ Indeed, for analysis-suitable T-splines, the refinement properties are already found in Ref. 34 for 2D respectively, in Ref. 33 for 3D.

Section 3 recalls the definition of hierarchical splines from Ref. 45, derives the canonical basis of the hierarchical spline space $\mathcal{X}_{\bullet} \subset H_{0}^{1}(\Omega)$ with Dirichlet boundary conditions (Sec. 3.3), formulates an admissibility criterion (3.20) for hierarchical meshes (Sec. 3.4), and introduces some local mesh-refinement rule for admissible hierarchical meshes (Sec. 3.5). One crucial observation is that the new mesh-refinement strategy for hierarchical meshes (Algorithm 3.1) guarantees that the number of (truncated) hierarchical B-splines on each element as well as the number of active elements contained in the support of each (truncated) hierarchical B-spline is uniformly bounded (Proposition [3.2). If one uses the strategy of Refs. 11 and 13 instead, this property is not satisfied for hierarchical B-splines, but only for truncated hierarchical B-splines. In general, the latter have a smaller, but also more complicated and not necessarily connected support.

The main result of Sec. 3 and the entire work is Theorem 3.1 which states that hierarchical splines together with the proposed local mesh-refinement strategy satisfy all assumptions of Sec.2, so that Theorem 2.1 applies. In particular, our work goes beyond Ref. 11 in two respects: while Ref. 11 only proves linear convergence of their adaptive algorithm, we give the first proof of optimal convergence rates for IGAFEM. We note that a preprint, 12 which appeared during the review process of this paper, proves optimal convergence if one applies the refinement strategy of Ref. 11 designed for truncated hierarchical splines. Moreover, both Refs. 11 and 12 adapt the analysis of Ref. 15 and are hence restricted to symmetric problems (i.e. $\mathbf{b}=0$ and $c \geq 0$ in (1.1) $)$. Our analysis exploits the framework of Ref. 14 together with some recent ideas from Refs. 21 and 7 and also covers the non-symmetric problem (1.1).

Technical contributions of general interest include the following: we prove that a hierarchical mesh is admissible if and only if it can be obtained by the meshrefinement strategy of Algorithm 3.1 (Proposition 5.1). Moreover, admissible meshes also allow a simpler computation of truncated hierarchical B-splines in the sense that truncation simplifies considerably (Proposition 5.2). Together with some ideas 
from Ref. 41, we use this observation to define a Scott-Zhang-type projector $J_{\bullet}: L^{2}(\Omega) \rightarrow \mathcal{X}_{\bullet}$ which is locally $L^{2}$ - and $H^{1}$-stable and has a first-order approximation property (Sec. 5.10).

\subsection{General notation}

Throughout, $|\cdot|$ denotes the absolute value of scalars, the Euclidean norm of vectors in $\mathbb{R}^{d}$, as well as the $d$-dimensional measure of a set in $\mathbb{R}^{d}$. Moreover, \# denotes the cardinality of a set as well as the multiplicity of a knot within a given knot vector. We write $A \lesssim B$ to abbreviate $A \leq c B$ with some generic constant $c>0$ which is clear from the context. Moreover, $A \simeq B$ abbreviates $A \lesssim B \lesssim A$. Throughout, mesh-related quantities have the same index, e.g. $\mathcal{X}_{\bullet}$ is the ansatz space corresponding to the mesh $\mathcal{T}_{\bullet}$. The analogous notation is used for meshes $\mathcal{T}_{\circ}, \mathcal{T}_{\star}$ or $\mathcal{T}_{\ell}$, etc. Moreover, we use $\widehat{\cdot}$ to transfer quantities in the physical domain $\Omega$ to the parameter domain $\widehat{\Omega}$, e.g. we write $\widehat{\mathbb{T}}$ for the set of all admissible meshes in the parameter domain instead of $\mathbb{T}$ which denotes the set of all admissible meshes in the physical domain.

\section{Axioms of Adaptivity (Revisited)}

The aim of this section is to formulate an adaptive algorithm (Algorithm 2.1) for conforming FEM discretizations of our model problem (1.1), where adaptivity is driven by the residual a posteriori error estimator (see (2.9) below). We identify the crucial properties of the underlying meshes, the mesh-refinement, as well as the finite element spaces which ensure that the residual error estimator fits into the general framework of Ref. 14 and which hence guarantee optimal convergence behavior of the adaptive algorithm. The main result of this section is Theorem 2.1 which is proved in Sec. 4 .

\subsection{Meshes}

Throughout, $\mathcal{T}_{\bullet}$ is a mesh of $\Omega$ in the following sense:

- $\mathcal{T}_{\bullet}$ is a finite set of compact Lipschitz domains;

- for all $T, T^{\prime} \in \mathcal{T}_{\bullet}$ with $T \neq T^{\prime}$, the intersection $T \cap T^{\prime}$ has measure zero;

- $\bar{\Omega}=\bigcup_{T \in \mathcal{T}_{\bullet}} T$, i.e. $\mathcal{T}_{\bullet}$ is a partition of $\Omega$.

We suppose that there is a countably infinite set $\mathbb{T}$ of admissible meshes. For $\mathcal{T}_{\bullet} \in \mathbb{T}$ and $\omega \subseteq \bar{\Omega}$, we define the patches of order $k \in \mathbb{N}_{0}$ inductively by

$$
\pi_{\bullet}^{0}(\omega):=\omega, \quad \pi_{\bullet}^{k}(\omega):=\bigcup\left\{T \in \mathcal{T}_{\bullet}: T \cap \pi_{\bullet}^{k-1}(\omega) \neq \emptyset\right\} .
$$

The corresponding set of elements is

$$
\Pi_{\bullet}^{k}(\omega):=\left\{T \in \mathcal{T}_{\bullet}: T \subseteq \pi_{\bullet}^{k}(\omega)\right\}, \quad \text { i.e. } \quad \pi_{\bullet}^{k}(\omega)=\bigcup \Pi_{\bullet}^{k}(\omega) .
$$

To abbreviate notation, we let $\pi_{\bullet}(\omega):=\pi_{\bullet}^{1}(\omega)$ and $\Pi_{\bullet}(\omega):=\Pi_{\bullet}^{1}(\omega)$. For $\mathcal{S}_{\bullet} \subseteq \mathcal{T}_{\bullet}$, we define $\pi_{\bullet}^{k}\left(\mathcal{S}_{\bullet}\right):=\pi_{\bullet}^{k}\left(\bigcup \mathcal{S}_{\bullet}\right)$ and $\Pi_{\bullet}^{k}\left(\mathcal{S}_{\bullet}\right):=\Pi_{\bullet}^{k}\left(\bigcup \mathcal{S}_{\bullet}\right)$. 
We suppose that there exist $C_{\text {shape }}, C_{\text {patch }}, C_{\text {trace }}, C_{\text {dual }}>0$ such that all meshes $\mathcal{T}_{\bullet} \in \mathbb{T}$ satisfy the following three properties (M1)-(M3):

(M1) Shape regularity. For all $T \in \mathcal{T}_{\bullet}$ and all $T^{\prime} \in \Pi_{\bullet}(T)$, it holds that

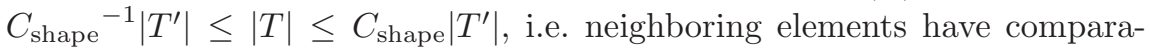
ble size.

(M2) Bounded element patch. For all $T \in \mathcal{T}_{\bullet}$, it holds that $\# \Pi_{\bullet}(T) \leq C_{\text {patch }}$, i.e. the number of elements in a patch is uniformly bounded.

(M3) Trace inequality. For all $T \in \mathcal{T}_{\bullet}$ and all $v \in H^{1}(\Omega)$, it holds that $\|v\|_{L^{2}(\partial T)}^{2} \leq C_{\text {trace }}\left(|T|^{-1 / d}\|v\|_{L^{2}(T)}^{2}+|T|^{1 / d}\|\nabla v\|_{L^{2}(T)}^{2}\right)$.

(M4) Local estimate in dual norm. For all $T \in \mathcal{T}_{\bullet}$ and all $w \in L^{2}(T)$, it holds that $|T|^{-1 / d}\|w\|_{H^{-1}(T)} \leq C_{\text {dual }}\|w\|_{L^{2}(T)}$, where $\|w\|_{H^{-1}(T)}:=$ $\sup \left\{\int_{T} w v d x: v \in H_{0}^{1}(T) \wedge\|\nabla v\|_{L^{2}(T)}=1\right\}$.

\subsection{Mesh-refinement}

For $\mathcal{T}_{\bullet} \in \mathbb{T}$ and an arbitrary set of marked elements $\mathcal{M}_{\bullet} \subseteq \mathcal{T}_{\bullet}$, we associate a corresponding refinement $\mathcal{T}_{0}:=\operatorname{refine}\left(\mathcal{T}_{\bullet}, \mathcal{M}_{\bullet}\right) \in \mathbb{T}$ with $\mathcal{M}_{\bullet} \subseteq \mathcal{T}_{\bullet} \backslash \mathcal{T}_{0}$, i.e. at least the marked elements have been refined. We define refine $\left(\mathcal{T}_{\bullet}\right)$ as the set of all $\mathcal{T}_{\circ}$ such that there exist meshes $\mathcal{T}_{(0)}, \ldots, \mathcal{T}_{(J)}$ and marked elements $\mathcal{M}_{(0)}, \ldots, \mathcal{M}_{(J-1)}$ with $\mathcal{T}_{\circ}=\mathcal{T}_{(J)}=\operatorname{refine}\left(\mathcal{T}_{(J-1)}, \mathcal{M}_{(J-1)}\right), \ldots, \mathcal{T}_{(1)}=\operatorname{refine}\left(\mathcal{T}_{(0)}, \mathcal{M}_{(0)}\right)$ and $\mathcal{T}_{(0)}=\mathcal{T}_{\bullet}$ Here, we formally allow $J=0$, i.e. $\mathcal{T}_{\bullet} \in \operatorname{refine}\left(\mathcal{T}_{\bullet}\right)$. We assume that there exists a fixed initial mesh $\mathcal{T}_{0} \in \mathbb{T}$ with $\mathbb{T}=\operatorname{refine}\left(\mathcal{T}_{0}\right)$.

We suppose that there exist $C_{\text {son }} \geq 2$ and $0<q_{\text {son }}<1$ such that all meshes $\mathcal{T}_{\bullet} \in$ $\mathbb{T}$ satisfy for arbitrary marked elements $\mathcal{M}_{\bullet} \subseteq \mathcal{T}_{\bullet}$ with corresponding refinement $\mathcal{T}_{\circ}:=\operatorname{refine}\left(\mathcal{T}_{\bullet}, \mathcal{M}_{\bullet}\right)$, the following elementary properties $(\mathrm{R} 1)-(\mathrm{R} 3)$ :

(R1) Bounded number of sons. It holds that $\# \mathcal{T}_{\circ} \leq C_{\text {son }} \# \mathcal{T}_{\bullet}$, i.e. one step of refinement leads to a bounded increase of elements.

(R2) Father is union of sons. It holds that $T=\bigcup\left\{T^{\prime} \in \mathcal{T}_{0}: T^{\prime} \subseteq T\right\}$ for all $T \in \mathcal{T}_{\bullet}$, i.e. each element $T$ is the union of its successors.

(R3) Reduction of sons. It holds that $\left|T^{\prime}\right| \leq q_{\text {son }}|T|$ for all $T \in \mathcal{T}_{\bullet}$ and all $T^{\prime} \in \mathcal{T}_{\circ}$ with $T^{\prime} \varsubsetneqq T$, i.e. successors are uniformly smaller than their father.

By induction and the definition of $\operatorname{refine}\left(\mathcal{T}_{\bullet}\right)$, one easily sees that $(\mathrm{R} 2)-(\mathrm{R} 3)$ remain valid if $\mathcal{T}_{\circ}$ is an arbitrary mesh in refine $\left(\mathcal{T}_{\bullet}\right)$. In particular, (R2)-(R3) imply that each refined element $T \in \mathcal{T}_{\bullet} \backslash \mathcal{T}_{0}$ is split into at least two sons, wherefore

$$
\#\left(\mathcal{T}_{\bullet} \backslash \mathcal{T}_{\circ}\right) \leq \# \mathcal{T}_{\circ}-\# \mathcal{T}_{\bullet} \quad \text { for all } \mathcal{T}_{\circ} \in \operatorname{refine}\left(\mathcal{T}_{\bullet}\right)
$$

Besides (R1)-(R3), we suppose the following less trivial requirements (R4)-(R5) with a generic constant $C_{\text {clos }}>0$ : 
(R4) Closure estimate. If $\mathcal{M}_{\ell} \subseteq \mathcal{T}_{\ell}$ and $\mathcal{T}_{\ell+1}=\operatorname{refine}\left(\mathcal{T}_{\ell}, \mathcal{M}_{\ell}\right)$ for all $\ell \in \mathbb{N}_{0}$, then

$$
\# \mathcal{T}_{L}-\# \mathcal{T}_{0} \leq C_{\text {clos }} \sum_{\ell=0}^{L-1} \# \mathcal{M}_{\ell} \quad \text { for all } L \in \mathbb{N} .
$$

(R5) Overlay estimate. For all $\mathcal{T}_{\bullet}, \mathcal{T}_{\star} \in \mathbb{T}$, there exists a common refinement $\mathcal{T}_{\circ} \in \operatorname{refine}\left(\mathcal{T}_{\bullet}\right) \cap \operatorname{refine}\left(\mathcal{T}_{\star}\right)$ such that

$$
\# \mathcal{T}_{\circ} \leq \# \mathcal{T}_{\bullet}+\# \mathcal{T}_{\star}-\# \mathcal{T}_{0}
$$

\subsection{Finite element space}

With each $\mathcal{T}_{\bullet} \in \mathbb{T}$, we associate a finite-dimensional space

$$
\mathcal{X}_{\bullet} \subset\left\{v \in H_{0}^{1}(\Omega):\left.v\right|_{T} \in H^{2}(T) \text { for all } T \in \mathcal{T}_{\bullet}\right\} \text {. }
$$

Let $U_{\bullet} \in \mathcal{X}_{\bullet}$ be the corresponding Galerkin approximation to the solution $u \in$ $H_{0}^{1}(\Omega)$, i.e.

$$
\left\langle U_{\bullet}, V_{\bullet}\right\rangle_{\mathcal{L}}=\int_{\Omega} f V_{\bullet} d x \quad \text { for all } V_{\bullet} \in \mathcal{X}_{\bullet}
$$

We note the Galerkin orthogonality

$$
\left\langle u-U_{\bullet}, V_{\bullet}\right\rangle_{\mathcal{L}}=0 \text { for all } V_{\bullet} \in \mathcal{X}_{\bullet}
$$

as well as the resulting Céa-type quasi-optimality

$$
\begin{aligned}
&\left\|u-U_{\bullet}\right\|_{H^{1}(\Omega)} \leq C_{\text {Céa }} \min _{V_{\bullet} \in \mathcal{X}_{\bullet}}\left\|u-V_{\bullet}\right\|_{H^{1}(\Omega)}, \\
& \text { with } \quad C_{\text {Céa }}:=\frac{\|\mathbf{A}\|_{L^{\infty}(\Omega)}+\|\mathbf{b}\|_{L^{\infty}(\Omega)}+\|c\|_{L^{\infty}(\Omega)}}{C_{\text {ell }}} .
\end{aligned}
$$

We suppose that there exist constants $C_{\text {inv }}>0$ and $k_{\text {loc }}, k_{\text {proj }} \in \mathbb{N}_{0}$ such that the following properties (S1)-(S3) hold for all $\mathcal{T}_{\bullet} \in \mathbb{T}$ :

(S1) Inverse estimate. For all $i, j \in\{0,1,2\}$ with $j \leq i$, all $V_{\bullet} \in \mathcal{X}_{\bullet}$ and all $T \in \mathcal{T}_{\bullet}$, it holds that $|T|^{(i-j) / d}\left\|V_{\bullet}\right\|_{H^{i}(T)} \leq C_{\text {inv }}\left\|V_{\bullet}\right\|_{H^{j}(T)}$.

(S2) Refinement guarantees nestedness. For all $\mathcal{T}_{0} \in \operatorname{refine}\left(\mathcal{T}_{\bullet}\right)$, it holds that $\mathcal{X}_{\bullet} \subseteq \mathcal{X}_{\circ}$.

(S3) Local domain of definition. With $\Pi_{\bullet}^{\text {loc }}:=\Pi_{\bullet}^{k_{\text {loc }}}, \pi_{\bullet}^{\text {loc }}:=\pi_{\bullet}^{k_{\text {loc }}}$ and $\pi_{\bullet}^{\text {proj }}:=$

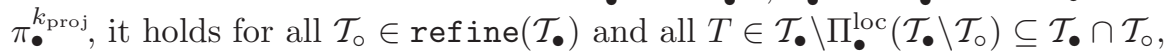
that $\left.V_{\circ}\right|_{\pi_{\bullet}^{\text {proj }}(T)} \in\left\{\left.V_{\bullet}\right|_{\pi_{\bullet}^{\text {proj }}(T)}: V_{\bullet} \in \mathcal{X}_{\bullet}\right\}$.

Besides (S1)-(S3), we suppose that there exist $C_{\mathrm{sz}}>0$ as well as $k_{\mathrm{app}}, k_{\mathrm{grad}} \in \mathbb{N}_{0}$ such that for all $\mathcal{T}_{\bullet} \in \mathbb{T}$, there exists a Scott-Zhang-type projector $J_{\bullet}: H_{0}^{1}(\Omega) \rightarrow \mathcal{X}$. with the following properties (S4)-(S6):

(S4) Local projection property. With $k_{\text {proj }} \in \mathbb{N}_{0}$ from (S3), let $\pi_{\bullet}^{\text {proj }}:=\pi_{\bullet}^{k_{\text {proj }}}$. For all $v \in H_{0}^{1}(\Omega)$ and $T \in \mathcal{T}_{\bullet}$, it holds that $\left.\left(J_{\bullet} v\right)\right|_{T}=\left.v\right|_{T}$, if $\left.v\right|_{\pi_{\bullet}^{\text {proj }}(T)} \in$ $\left\{\left.V_{\bullet}\right|_{\pi_{\bullet}^{\mathrm{proj}}(T)}: V_{\bullet} \in \mathcal{X}_{\bullet}\right\}$. 
(S5) Local $\boldsymbol{L}^{2}$-approximation property. Let $\pi_{\bullet}^{\text {app }}:=\pi_{\bullet}^{k_{\text {app }}}$. For all $T \in \mathcal{T}_{\bullet}$ and all $v \in H_{0}^{1}(\Omega)$, it holds that $\left\|\left(1-J_{\bullet}\right) v\right\|_{L^{2}(T)} \leq C_{\mathrm{sZ}}|T|^{1 / d}\|v\|_{H^{1}\left(\pi_{\bullet}^{\mathrm{app}}(T)\right)}$.

(S6) Local $\boldsymbol{H}^{1}$-stability. Let $\pi_{\bullet}^{\text {grad }}:=\pi_{\bullet}^{k_{\text {grad }}}$. For all $T \in \mathcal{T}_{\bullet}$ and $v \in H_{0}^{1}(\Omega)$, it holds that $\left\|\nabla J_{\bullet} v\right\|_{L^{2}(T)} \leq C_{\mathrm{sz}}\|v\|_{H^{1}\left(\pi_{\bullet}^{\operatorname{grad}}(T)\right)}$.

\subsection{Error estimator}

Let $\mathcal{T}_{\bullet} \in \mathbb{T}$ and $T_{1} \in \mathcal{T}_{\bullet}$. For almost every $x \in \partial T_{1} \cap \Omega$, there exists a unique element $T_{2} \in \mathcal{T}_{\bullet}$ with $x \in T_{1} \cap T_{2}$. We denote the corresponding outer normal vectors by $\nu_{1}$ respectively, $\nu_{2}$ and define the normal jump as

$$
\left[\mathbf{A} \nabla U_{\bullet} \cdot \nu\right](x)=\left.\mathbf{A} \nabla U_{\bullet}\right|_{T_{1}}(x) \cdot \nu_{1}(x)+\left.\mathbf{A} \nabla U_{\bullet}\right|_{T_{2}}(x) \cdot \nu_{2}(x) .
$$

With this definition, we employ the residual a posteriori error estimator

$$
\eta_{\bullet}:=\eta_{\bullet}\left(\mathcal{T}_{\bullet}\right) \quad \text { with } \quad \eta_{\bullet}\left(\mathcal{S}_{\bullet}\right)^{2}:=\sum_{T \in \mathcal{S}_{\bullet}} \eta_{\bullet}(T)^{2} \quad \text { for all } \mathcal{S}_{\bullet} \subseteq \mathcal{T}_{\bullet}
$$

where, for all $T \in \mathcal{T}_{\bullet}$, the local refinement indicators read

$$
\begin{aligned}
\eta_{\bullet}(T)^{2}:= & |T|^{2 / d}\left\|f+\operatorname{div} \mathbf{A} \nabla U_{\bullet}-\mathbf{b} \cdot \nabla U_{\bullet}-c U_{\bullet}\right\|_{L^{2}(T)}^{2} \\
& +|T|^{1 / d}\left\|\left[\mathbf{A} \nabla U_{\bullet} \cdot \nu\right]\right\|_{L^{2}(\partial T \cap \Omega)}^{2} .
\end{aligned}
$$

We refer, e.g. Refs. 1 and 44 for the analysis of the residual a posteriori error estimator 2.9) in the frame of standard FEM with piecewise polynomials of fixed order.

Remark 2.1. If $\mathcal{X}_{\bullet} \subset C^{1}(\bar{\Omega})$, then the jump contributions in (2.9) vanish and $\eta \bullet(T)$ consists only of the volume residual; see Refs. 11 and 12 in the frame of IGAFEM.

\subsection{Data oscillations}

The definition of the data oscillations corresponding to the residual error estimator (2.9) requires some further notation. Let $\mathcal{P}(\Omega) \subset H^{1}(\Omega)$ be a fixed discrete subspace. We suppose that there exists $C_{\text {inv }}^{\prime}$ such that the following property (O1) holds for all $\mathcal{T}_{\bullet} \in \mathbb{T}$ :

(O1) Inverse estimate in dual norm. For all $W \in \mathcal{P}(\Omega)$, it holds that $|T|^{1 / d}\|W\|_{L^{2}(T)} \leq C_{\text {inv }}^{\prime}\|W\|_{H^{-1}(T)}$, where $\|W\|_{H^{-1}(T)}:=\sup \left\{\int_{T} W v d x: v \in\right.$ $\left.H_{0}^{1}(T) \wedge\|\nabla v\|_{L^{2}(T)}=1\right\}$.

Besides (O1), we suppose that there exists $C_{\text {lift }}>0$ such that for all $\mathcal{T}_{\bullet} \in$ $\mathbb{T}$ and all $T, T^{\prime} \in \mathcal{T}_{\bullet}$ with $(d-1)$-dimensional intersection $E:=T \cap T^{\prime}$, there exists a lifting operator $L_{\bullet}, E:\left\{\left.W\right|_{E}: W \in \mathcal{P}(\Omega)\right\} \rightarrow H_{0}^{1}\left(T \cup T^{\prime}\right)$ with the following properties $(\mathrm{O} 2)-(\mathrm{O} 4)$ : 
(O2) Lifting inequality. For all $W \in \mathcal{P}(\Omega)$, it holds that $\int_{E} W^{2} d x \leq$ $C_{\text {lift }} \int_{E} L_{\bullet}, E\left(\left.W\right|_{E}\right) W d x$.

(O3) $\boldsymbol{L}^{2}$-control. For all $W \in \mathcal{P}(\Omega)$, it holds that $\left\|L_{\bullet, E}\left(\left.W\right|_{E}\right)\right\|_{L^{2}\left(T \cup T^{\prime}\right)}^{2} \leq$ $C_{\text {lift }}\left|T \cup T^{\prime}\right|^{1 / d}\|W\|_{L^{2}(E)}^{2}$.

(O4) $\boldsymbol{H}^{1}$-control. For all $W \in \mathcal{P}(\Omega)$, it holds that $\left\|\nabla L_{\bullet, E}\left(\left.W\right|_{E}\right)\right\|_{L^{2}\left(T \cup T^{\prime}\right)}^{2} \leq$ $C_{\text {lift }}\left|T \cup T^{\prime}\right|^{-1 / d}\|W\|_{L^{2}(E)}^{2}$.

Let $\mathcal{T}_{\bullet} \in \mathbb{T}$. For $T \in \mathcal{T}_{\bullet}$, we define the $L^{2}$-orthogonal projection $P_{\bullet}, T: L^{2}(T) \rightarrow$ $\left\{\left.W\right|_{T}: W \in \mathcal{P}(\Omega)\right\}$. For an interior edge $E \in \mathcal{E}_{\bullet}, T:=\left\{T \cap T^{\prime}: T^{\prime} \in \mathcal{T}_{\bullet} \wedge \operatorname{dim}(T \cap\right.$ $\left.\left.T^{\prime}\right)=d-1\right\}$, we define the $L^{2}$-orthogonal projection $P_{\bullet}, E: L^{2}(E) \rightarrow\left\{\left.W\right|_{E}: W \in\right.$ $\mathcal{P}(\Omega)\}$. Note that $\cup \mathcal{E}_{\bullet}, T=\overline{\partial T \cap \Omega}$. For $V_{\bullet} \in \mathcal{X}_{\bullet}$, we define the corresponding oscillations

$$
\operatorname{osc}_{\bullet}\left(V_{\bullet}\right):=\operatorname{osc} \bullet\left(V_{\bullet}, \mathcal{T}_{\bullet}\right) \quad \text { with } \quad \operatorname{osc}_{\bullet}\left(V_{\bullet}, \mathcal{S}_{\bullet}\right)^{2}:=\sum_{T \in \mathcal{S}_{\bullet}} \operatorname{osc}_{\bullet}\left(V_{\bullet}, T\right)^{2}
$$

for all $\mathcal{S}_{\bullet} \subseteq \mathcal{T}_{\bullet}$,

where, for all $T \in \mathcal{T}_{\bullet}$, the local oscillations read

$$
\begin{aligned}
\operatorname{Osc}_{\bullet}\left(V_{\bullet}, T\right)^{2}:= & |T|^{2 / d}\left\|\left(1-P_{\bullet}, T\right)\left(f+\operatorname{div} \mathbf{A} \nabla V_{\bullet}-\mathbf{b} \cdot \nabla V_{\bullet}-c V_{\bullet}\right)\right\|_{L^{2}(T)}^{2} \\
& +\sum_{E \in \mathcal{E}_{\bullet}, T}|T|^{1 / d}\left\|\left(1-P_{\bullet}, E\right)\left[\mathbf{A} \nabla V_{\bullet} \cdot \nu\right]\right\|_{L^{2}(E)}^{2} .
\end{aligned}
$$

We refer, e.g. to Ref. 36 for the analysis of oscillations in the frame of standard FEM with piecewise polynomials of fixed order.

Remark 2.2. If $\mathcal{X}_{\bullet} \subset C^{1}(\bar{\Omega})$, then the jump contributions in (2.10) vanish and $\operatorname{Osc}_{\bullet}\left(V_{\bullet}, T\right)$ consists only of the volume oscillations; see Refs. 11 and 12 in the frame of IGAFEM.

\subsection{Adaptive algorithm}

We consider the common formulation of an adaptive mesh-refining algorithm; see, e.g. Algorithm 2.2 of Ref. 14

Algorithm 2.1. Input: Adaptivity parameter $0<\theta \leq 1$ and marking constant $C_{\min } \geq 1$.

Loop: For each $\ell=0,1,2, \ldots$, iterate the following Steps (i)-(iv):

(i) Compute Galerkin approximation $U_{\ell} \in \mathcal{X}_{\ell}$.

(ii) Compute refinement indicators $\eta_{\ell}(T)$ for all elements $T \in \mathcal{T}_{\ell}$.

(iii) Determine a set of marked elements $\mathcal{M}_{\ell} \subseteq \mathcal{T}_{\ell}$ which has up to the multiplicative constant $C_{\min }$ minimal cardinality such that $\theta \eta_{\ell}^{2} \leq \eta_{\ell}\left(\mathcal{M}_{\ell}\right)^{2}$.

(iv) Generate refined mesh $\mathcal{T}_{\ell+1}:=\operatorname{refine}\left(\mathcal{T}_{\ell}, \mathcal{M}_{\ell}\right)$.

Output: Refined meshes $\mathcal{T}_{\ell}$ and corresponding Galerkin approximations $U_{\ell}$ with error estimators $\eta_{\ell}$ for all $\ell \in \mathbb{N}_{0}$. 


\subsection{Main theorem on rate optimal convergence}

We define

$$
\mathbb{T}(N):=\left\{\mathcal{T}_{\bullet} \in \mathbb{T}: \# \mathcal{T}_{\bullet}-\# \mathcal{T}_{0} \leq N\right\} \quad \text { for all } N \in \mathbb{N}_{0}
$$

and for all $s>0$ :

$$
\|u\|_{\mathbb{A}_{s}}:=\sup _{N \in \mathbb{N}_{0}} \min _{\mathcal{T}_{\bullet} \in \mathbb{T}(N)}(N+1)^{s} \eta_{\bullet} \in[0, \infty]
$$

and

$$
\|u\|_{\mathbb{B}_{s}}:=\sup _{N \in \mathbb{N}_{0}}\left(\min _{\mathcal{T}_{\bullet} \in \mathbb{T}(N)}(N+1)^{s} \inf _{V_{\bullet} \in \mathcal{X}_{\bullet}}\left(\left\|u-V_{\bullet}\right\|_{H^{1}(\Omega)}+\operatorname{osc}_{\bullet}\left(V_{\bullet}\right)\right)\right) \in[0, \infty] .
$$

By definition, $\|u\|_{\mathbb{A}_{s}}<\infty$ (respectively $\|u\|_{\mathbb{B}_{s}}<\infty$ ) implies that the error estimator $\eta_{\bullet}$ (respectively the total error) on the optimal meshes $\mathcal{T}_{\bullet}$ decays at least with rate $\mathcal{O}\left(\left(\# \mathcal{T}_{\bullet}\right)^{-s}\right)$. The following main theorem states that each possible rate $s>0$ is in fact realized by Algorithm 2.1. The (sketch of the) proof is given in Sec. 4. It is split into eight steps and builds upon the analysis of Ref. 14.

Theorem 2.1. There hold the following four points:

(i) Suppose (M2)-(M3) and (S5)-(S6). Then, the residual error estimator (2.9) satisfies reliability, i.e. there exists a constant $C_{\mathrm{rel}}>0$ such that

$$
\left\|u-U_{\bullet}\right\|_{H^{1}(\Omega)}+\mathrm{osc}_{\bullet} \leq C_{\mathrm{rel}} \eta_{\bullet} \quad \text { for all } \mathcal{T}_{\bullet} \in \mathbb{T} .
$$

(ii) Suppose (M1)-(M4), (S1) and (O1)-(O4). Then, the residual error estimator satisfies efficiency, i.e. there exists a constant $C_{\mathrm{eff}}>0$ such that

$$
C_{\mathrm{eff}}^{-1} \eta_{\bullet} \leq \inf _{V_{\bullet} \in \mathcal{X}_{\bullet}}\left(\left\|u-V_{\bullet}\right\|_{H^{1}(\Omega)}+\operatorname{osc}_{\bullet}\left(V_{\bullet}\right)\right) .
$$

(iii) Suppose (M1)-(M3), (R2)-(R3), (S1)-(S2) and (S5)-(S6). Then, for arbitrary $0<\theta \leq 1$ and $C_{\min } \geq 1$, there exist constants $C_{\text {lin }}>0$ and $0<q_{\text {lin }}<1$ such that the estimator sequence of Algorithm 2.1 guarantees linear convergence in the sense of

$$
\eta_{\ell+j}^{2} \leq C_{\operatorname{lin}} q_{\text {lin }}{ }^{j} \eta_{\ell}^{2} \quad \text { for all } j, \ell \in \mathbb{N}_{0} .
$$

(iv) Suppose (M1)-(M3), (R1)-(R5) and (S1)-(S6). Then, there exists a constant $0<\theta_{\text {opt }} \leq 1$ such that for all $0<\theta<\theta_{\text {opt }}$, all $C_{\min } \geq 1$, and all $s>0$, there exist constants $c_{\mathrm{opt}}, C_{\mathrm{opt}}>0$ such that

$$
c_{\mathrm{opt}}\|u\|_{\mathbb{A}_{s}} \leq \sup _{\ell \in \mathbb{N}_{0}}\left(\# \mathcal{T}_{\ell}-\# \mathcal{T}_{0}+1\right)^{s} \eta_{\ell} \leq C_{\mathrm{opt}}\|u\|_{\mathbb{A}_{s}},
$$

i.e. the estimator sequence will decay with each possible rate $s>0$. 
All involved constants $C_{\mathrm{rel}}, C_{\mathrm{eff}}, C_{\mathrm{lin}}, q_{\mathrm{lin}}, \theta_{\mathrm{opt}}, c_{\mathrm{opt}}$ and $C_{\mathrm{opt}}$ depend only on the assumptions made as well as the coefficients of the differential operator $\mathcal{L}$ and $\operatorname{diam}(\Omega)$, where $C_{\text {lin }}, q_{\text {lin }}$ depend additionally on $\theta$ and the sequence $\left(U_{\ell}\right)_{\ell \in \mathbb{N}_{0}}$, and $c_{\mathrm{opt}}, C_{\mathrm{opt}}$ depend furthermore on $C_{\mathrm{min}}$, and $s>0$.

Remark 2.3. If the assumptions of Theorem 2.1(i)-(ii) are satisfied, there holds in particular

$$
C_{\text {eff }}^{-1}\|u\|_{\mathbb{A}_{s}} \leq\|u\|_{\mathbb{B}_{s}} \leq C_{\text {rel }}\|u\|_{\mathbb{A}_{s}} \text { for all } s>0
$$

Remark 2.4. Note that almost minimal cardinality of $\mathcal{M}_{\ell}$ in Step (iii) of Algorithm 2.1 is only required to prove optimal convergence behavior (2.17), while linear convergence (2.16) formally allows $C_{\min }=\infty$, i.e. it suffices that $\mathcal{M}_{\ell}$ satisfies the Dörfler marking criterion in Step (iii). We refer to Secs. 4.3-4.4 of Ref.14 for details.

Remark 2.5. (a) If the bilinear form $\langle\cdot, \cdot\rangle_{\mathcal{L}}$ is symmetric, $C_{\text {lin }}, q_{\text {lin }}$ as well as $c_{\mathrm{opt}}$, $C_{\text {opt }}$ are then independent of $\left(U_{\ell}\right)_{\ell \in \mathbb{N}_{0}}$; see Remark 4.1 below.

(b) If the bilinear form $\langle\cdot, \cdot\rangle_{\mathcal{L}}$ is non-symmetric, there exists an index $\ell_{0} \in \mathbb{N}_{0}$ such that the constants $C_{\text {lin }}, q_{\text {lin }}$ as well as $c_{\text {opt }}, C_{\text {opt }}$ are independent of $\left(U_{\ell}\right)_{\ell \in \mathbb{N}_{0}}$, if (2.16)-(2.17) are formulated only for $\ell \geq \ell_{0}$. We refer to Theorem 19 of Ref. 7 .

Remark 2.6. If $\mathcal{X}_{\bullet} \subset C^{1}(\bar{\Omega})$, all jump contributions vanish; see Remark 2.1] and Remark 2.2. In this case, the assumptions (O2)-(O4) are not necessary for the proof of (2.15).

Remark 2.7. (a) Let $h_{\ell}:=\max _{T \in \mathcal{T}_{\ell}}|T|^{1 / d}$ be the maximal mesh-width. Then, $h_{\ell} \rightarrow 0$ as $\ell \rightarrow \infty$, ensures that $\mathcal{X}_{\infty}:=\overline{\bigcup_{\ell \in \mathbb{N}_{0}} \mathcal{X}_{\ell}}=H_{0}^{1}(\Omega)$. To see this, recall that (S2) ensures that $\bigcup_{\ell \in \mathbb{N}_{0}} \mathcal{X}_{\ell}$ is a vector space and, in particular, convex. By Mazur's lemma (see, e.g. Theorem 3.12 of Ref. 37), it is thus sufficient to show that $\bigcup_{\ell \in \mathbb{N}_{0}} \mathcal{X}_{\ell}$ is weakly dense in $H_{0}^{1}(\Omega)$. Let $v \in H_{0}^{1}(\Omega)$. The Banach-Alaoglu theorem (see, e.g. Theorem 3.15 of Ref. 37) together with (M2) and (S5)-(S6) proves that each subsequence $\left(J_{\ell_{m}} v\right)_{m \in \mathbb{N}_{0}}$ admits a further subsequence $\left(J_{\ell_{m_{n}}} v\right)_{n \in \mathbb{N}_{0}}$ which is weakly convergent in $H_{0}^{1}(\Omega)$ towards some limit $w \in H_{0}^{1}(\Omega)$. The Rellich compactness theorem hence implies $\left\|w-J_{\ell_{m_{n}}} v\right\|_{L^{2}(\Omega)} \rightarrow 0$ as $n \rightarrow \infty$. On the other hand, (S5) together with (M2), (R1)-(R3), and $h_{\ell} \rightarrow 0$ shows that $\left\|v-J_{\ell} v\right\|_{L^{2}(\Omega)} \lesssim h_{\ell}\|v\|_{H^{1}(\Omega)} \rightarrow 0$ as $\ell \rightarrow \infty$. Together with the uniqueness of limits, these two observations conclude $v=w$. Overall, each subsequence $\left(J_{\ell_{m}} v\right)_{m \in \mathbb{N}_{0}}$ of $\left(J_{\ell} v\right)_{\ell \in \mathbb{N}}$ admits a further subsequence $\left(J_{\ell_{m_{n}}} v\right)_{n \in \mathbb{N}_{0}}$ which converges weakly in $H_{0}^{1}(\Omega)$ to $v$. Basic calculus thus yields that $J_{\ell} v \rightarrow v$ weakly in $H_{0}^{1}(\Omega)$ as $\ell \rightarrow \infty$. This concludes the proof.

(b) We note that the latter observation allows to follow the ideas of Ref. 7 and to show that the adaptive algorithm yields convergence even if the bilinear form $\langle\cdot, \cdot\rangle_{\mathcal{L}}$ is only elliptic up to some compact perturbation, provided that the continuous 
problem is well-posed. This includes, e.g. adaptive FEM for the Helmhotz equation. For details, the reader is referred to Ref. [7]

\section{Hierarchical Setting}

In this section, we recall the definition of hierarchical (B-)splines from Ref. 45 and propose a local mesh-refinement strategy. The main result of this section is Theorem 3.1 which states that hierarchical splines together with the proposed meshrefinement strategy fit into the abstract setting of Sec. 2 and are hence covered by Theorem 2.1 The proof of Theorem 3.1 is given in Sec. 5

\subsection{Nested tensor meshes and splines}

We define the parameter domain $\widehat{\Omega}:=(0,1)^{d}$. Let $p_{1}, \ldots, p_{d} \geq 1$ be fixed polynomial degrees with $p:=\max _{i=1, \ldots, d} p_{i}$. Let $\widehat{\mathcal{K}}^{0}$ be an arbitrary fixed $d$-dimensional vector of $p_{i}$-open knot vectors with multiplicity smaller or equal to $p_{i}$ for the interior knots, i.e.

$$
\widehat{\mathcal{K}}^{0}=\left(\widehat{\mathcal{K}}_{1}^{0}, \ldots, \widehat{\mathcal{K}}_{d}^{0}\right)
$$

where $\widehat{\mathcal{K}}_{i}^{0}=\left(t_{i, j}^{0}\right)_{j=0}^{N_{i}^{0}+p_{i}}$ is a non-decreasing vector in $[0,1]$ such that $t_{i, 0}^{0}=\cdots=$ $t_{i, p_{i}}^{0}=0, t_{i, N_{i}^{0}}^{0}=\cdots=t_{i, N_{i}^{0}+p_{i}}^{0}$, and $\# t_{i, j}^{0}:=\#\left\{k \in\left\{0, \ldots, N_{i}^{0}+p_{i}\right\}: t_{i, k}^{0}=t_{i, j}^{0}\right\} \leq$ $p_{i}$ for $j=p_{i}+1, \ldots, N_{i}^{0}-1$. For $k \in \mathbb{N}_{0}$, we recursively define $\widehat{\mathcal{K}}^{k+1}$ as the uniform $h$-refinement of $\widehat{\mathcal{K}}^{k}$, i.e. it is obtained by inserting the knot $\frac{t_{i, j}^{k}+t_{i, j+1}^{k}}{2}$ of multiplicity one in each knot span $\left[t_{i, j}^{k}, t_{i, j+1}^{k}\right]$ with $t_{i, j}^{k} \neq t_{i, j+1}^{k}$. Let $\widehat{\mathcal{B}}^{k}$ be the corresponding tensor-product B-spline basis, i.e.

$$
\widehat{\mathcal{B}}^{k}=\left\{\widehat{B}_{j_{1}, \ldots, j_{d}}^{k}: j_{i} \in\left\{1, \ldots, N_{i}^{k}\right\}\right\},
$$

where for $\left(s_{1}, \ldots, s_{d}\right) \in \mathbb{R}^{d}$

$$
\widehat{B}_{j_{1}, \ldots, j_{d}}^{k}\left(s_{1}, \ldots, s_{d}\right):=\prod_{i=1}^{d} \widehat{B}\left(s_{i} \mid t_{i, j_{i}-1}^{k}, \ldots, t_{i, j_{i}+p_{i}}^{k}\right),
$$

where $\widehat{B}\left(\cdot \mid t_{i, j_{i}-1}^{k}, \ldots, t_{i, j_{i}+p_{i}}^{k}\right)$ denotes the one-dimensional B-spline corresponding to the local knot vector $\left(t_{i, j_{i}-1}^{k}, \ldots, t_{i, j_{i}+p_{i}}^{k}\right)$. It is well known that the function in $\widehat{\mathcal{B}}^{k}$ have $\operatorname{support} \operatorname{supp}\left(\widehat{B}_{j_{1}, \ldots, j_{d}}^{k}\right)=\left[t_{1, j_{1}-1}^{k}, t_{1, j_{1}+p_{1}}^{k}\right] \times \cdots \times\left[t_{d, j_{d}-1}^{k}, t_{d, j_{d}+p_{d}}^{k}\right] \subseteq[0,1]^{d}$, form a partition of unity, and are even locally linearly independent, i.e. for any open set $O \subseteq[0,1]^{d}$, the restricted B-splines $\left\{\left.\widehat{\beta}\right|_{O}: \widehat{\beta} \in \widehat{\mathcal{B}}^{k} \wedge \operatorname{supp}(\widehat{\beta}) \cap O \neq \emptyset\right\}$ are linearly independent. Let $\widehat{\mathcal{Y}}^{k}:=\operatorname{span}\left(\widehat{\mathcal{B}}^{k}\right)$. This yields a nested sequence of tensor-product $d$-variate spline function spaces $\left(\widehat{\mathcal{Y}}^{k}\right)_{k \in \mathbb{N}_{0}}$ that are at least Lipschitz continuous

$$
\widehat{\mathcal{Y}}^{k} \subset \widehat{\mathcal{Y}}^{k+1} \subset W^{1, \infty}(\widehat{\Omega}) .
$$

In particular, each $\widehat{\beta}^{k} \in \widehat{\mathcal{B}}^{k}$ can be written as linear combination of functions in $\widehat{\mathcal{B}}^{k+1}$, i.e. it has a unique representation of the form

$$
\widehat{\beta}^{k}=\sum_{\widehat{\beta}^{k+1} \in \widehat{\mathcal{B}}^{k+1}} c_{\widehat{\beta}^{k+1}} \widehat{\beta}^{k+1} .
$$


By the knot insertion procedure, one can show that these coefficients are nonnegative, i.e. $c_{\widehat{\beta}^{k+1}} \geq 0$; see, e.g. Sec. 2.1.3 of Ref. 6. With

$$
\begin{gathered}
\widehat{T}_{j_{1}, \ldots, j_{d}}^{k}:=\left[t_{1, j_{1}-1}^{k}, t_{1, j_{1}}^{k}\right] \times \cdots \times\left[t_{d, j_{d}-1}^{k}, t_{d, j_{d}}^{k}\right] \quad \text { for all } j_{i}=1, \ldots, N_{i}^{k}+p_{i}, \\
i=1, \ldots, d,
\end{gathered}
$$

we define the corresponding set of all non-trivial closed cells of level $k$ as

$$
\widehat{\mathcal{T}}^{k}:=\left\{\widehat{T}_{j_{1}, \ldots, j_{d}}^{k}:\left|\widehat{T}_{j_{1}, \ldots, j_{d}}^{k}\right|>0 \wedge j_{i}=1, \ldots, N_{i}^{k}+p_{i} \wedge i=1, \ldots, d\right\} .
$$

Each function in $\widehat{\mathcal{Y}}^{k}$ is a $\widehat{\mathcal{T}}^{k}$ piecewise polynomial, where the smoothness across the boundary of an element $\widehat{T}$ depends only on the corresponding knot multiplicities. For a more detailed presentation of tensor-product splines, we refer to, e.g. Refs. 17, 39 and 6 .

\subsection{Hierarchical meshes and splines in the parameter domain $\widehat{\mathbf{\Omega}}$}

Meshes $\mathcal{T}_{\bullet}$ and corresponding spaces $\mathcal{X}_{\bullet}$ are defined through their counterparts on the parameter domain $\widehat{\Omega}:=(0,1)^{d}$. Let $\left(\widehat{\Omega}_{\bullet}^{k}\right)_{k \in \mathbb{N}_{0}}$ be a nested sequence of closed subsets of $\widehat{\widehat{\Omega}}=[0,1]^{d}$ such that

$$
\widehat{\Omega}_{\bullet}^{0}=\overline{\widehat{\Omega}} \text { and } \widehat{\Omega}_{\bullet}^{k} \supseteq \widehat{\Omega}_{\bullet}^{k+1} .
$$

We suppose that for $k>0$ each $\widehat{\Omega}_{\bullet}^{k}$ is the union of a selection of cells of level $k-1$, i.e.

$$
\widehat{\Omega}_{\bullet}^{k}=\bigcup\left\{\widehat{T} \in \widehat{\mathcal{T}}^{k-1}: \widehat{T} \subseteq \widehat{\Omega}_{\bullet}^{k}\right\}
$$

Moreover, we assume the existence of some minimal $M_{\bullet}>0$ such that $\widehat{\Omega}_{\bullet}^{M}=\emptyset$. Then, we define the mesh in the parameter domain

$$
\widehat{\mathcal{T}}_{\bullet}:=\bigcup_{k \in \mathbb{N}_{0}}\left\{\widehat{T} \in \widehat{\mathcal{T}}^{k}: \widehat{T} \subseteq \widehat{\Omega}_{\bullet}^{k} \wedge \widehat{T} \not \widehat{\Omega}_{\bullet}^{k+1}\right\} ;
$$

see Fig. 1 for an illustrative example.

Note that $\widehat{\mathcal{T}}^{k} \cap \widehat{\mathcal{T}}^{k^{\prime}}=\emptyset$ for $k \neq k^{\prime} \in \mathbb{N}_{0}$. For $\widehat{T} \in \widehat{\mathcal{T}}_{\bullet}$, there exists a unique $\operatorname{level}(\widehat{T}):=k \in \mathbb{N}_{0}$ with $\widehat{T} \subseteq \widehat{\Omega}_{\bullet}^{k}$ and $\widehat{T} \not \subset \widehat{\Omega}_{\bullet}^{k+1}$. Note that $\widehat{\mathcal{T}}_{\bullet}$ is a mesh of $\widehat{\Omega}$ in the sense of Sec. 2.1

With these preparations, one inductively defines the set of all hierarchical Bsplines in the parameter domain $\widehat{\mathcal{H}}_{\bullet}:=\widehat{\mathcal{H}}_{\bullet}^{M \bullet-1}$ as follows:

(i) Define $\widehat{\mathcal{H}}_{\bullet}^{0}:=\widehat{\mathcal{B}}^{0}$.

(ii) For $k=0, \ldots, M_{\bullet}-2$, define $\widehat{\mathcal{H}}_{\bullet}^{k+1}:=\operatorname{old}\left(\widehat{\mathcal{H}}_{\bullet}^{k+1}\right) \cup \operatorname{new}\left(\widehat{\mathcal{H}}_{\bullet}^{k+1}\right)$, where:

$$
\begin{aligned}
\operatorname{old}\left(\widehat{\mathcal{H}}_{\bullet}^{k+1}\right) & :=\left\{\widehat{\beta} \in \widehat{\mathcal{H}}_{\bullet}^{k}: \operatorname{supp}(\widehat{\beta}) \nsubseteq \widehat{\Omega}_{\bullet}^{k+1}\right\}, \\
\operatorname{new}\left(\widehat{\mathcal{H}}_{\bullet}^{k+1}\right): & =\left\{\widehat{\beta} \in \widehat{\mathcal{B}}^{k+1}: \operatorname{supp}(\widehat{\beta}) \subseteq \widehat{\Omega}_{\bullet}^{k+1}\right\} .
\end{aligned}
$$




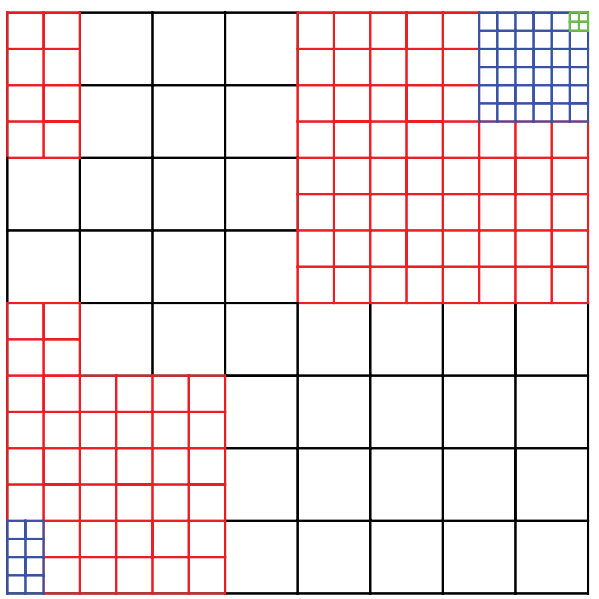

Fig. 1. (Color online) A two-dimensional hierarchical mesh $\widehat{\mathcal{T}}_{\bullet}$ on the parameter domain is depicted, where $\widehat{\Omega}_{\bullet}^{4}=\emptyset$. The corresponding domains $\widehat{\Omega}_{\bullet}^{0} \supseteq \widehat{\Omega}_{\bullet}^{1} \supseteq \widehat{\Omega}_{\bullet}^{2} \supseteq \widehat{\Omega}_{\bullet}^{3}$ are highlighted in black, red, blue and green.

One can prove that the so-called hierarchical basis $\widehat{\mathcal{H}}_{\bullet}$ is linearly independent; see Lemma 2 of Ref. 45. By definition, it holds that

$$
\widehat{\mathcal{H}}_{\bullet}=\bigcup_{k \in \mathbb{N}_{0}}\left\{\widehat{\beta} \in \widehat{\mathcal{B}}^{k}: \operatorname{supp}(\widehat{\beta}) \subseteq \widehat{\Omega}_{\bullet}^{k} \wedge \operatorname{supp}(\widehat{\beta}) \not \widehat{\Omega}_{\bullet}^{k+1}\right\} .
$$

Note that $\widehat{\mathcal{B}}^{k} \cap \widehat{\mathcal{B}}^{k^{\prime}}=\emptyset$ for $k \neq k^{\prime} \in \mathbb{N}_{0}$. For $\widehat{\beta} \in \widehat{\mathcal{H}}_{\bullet}$, there exists a unique $\operatorname{level}(\widehat{\beta}):=k \in \mathbb{N}_{0}$ with $\operatorname{supp}(\widehat{\beta}) \subseteq \widehat{\Omega}_{\bullet}^{k}$ and $\operatorname{supp}(\widehat{\beta}) \nsubseteq \widehat{\Omega}_{\bullet}^{k+1}$.

The hierarchical basis $\widehat{\mathcal{H}}_{\bullet}$ and the mesh $\widehat{\mathcal{T}}_{\bullet}$ are compatible in the following sense: for all $\widehat{\beta} \in \widehat{\mathcal{H}}_{\bullet}$, the corresponding support can be written as union of elements in $\widehat{\mathcal{T}}^{\text {level }(\widehat{\beta})}$, i.e.

$$
\operatorname{supp}(\widehat{\beta})=\bigcup\left\{\widehat{T} \in \widehat{\mathcal{T}}^{\operatorname{level}(\widehat{\beta})}: \widehat{T} \subseteq \operatorname{supp}(\widehat{\beta})\right\}
$$

Each such element $\widehat{T} \in \widehat{\mathcal{T}}^{\operatorname{level}(\widehat{\beta})}$ with $\widehat{T} \subseteq \operatorname{supp}(\widehat{\beta}) \subseteq \widehat{\Omega}_{\bullet}^{\operatorname{level}(\widehat{\beta})}$ satisfies $\widehat{T} \in \widehat{\mathcal{T}}_{\bullet}$ or $\widehat{T} \subseteq \widehat{\Omega}_{\bullet}^{\operatorname{level}(\widehat{\beta})+1}$. In either case, we see that $\widehat{T}$ can be written as union of elements in $\widehat{\mathcal{T}}_{\bullet}$ with level greater or equal to $\operatorname{level}(\widehat{\beta})$. Altogether, we have

$$
\operatorname{supp}(\widehat{\beta})=\bigcup_{k \geq \operatorname{level}(\widehat{\beta})}\left\{\widehat{T} \in \widehat{\mathcal{T}}_{\bullet} \cap \widehat{\mathcal{T}}^{k}: \widehat{T} \subseteq \operatorname{supp}(\widehat{\beta})\right\}
$$

Moreover, $\operatorname{supp}(\widehat{\beta})$ must contain at least one element of level $(\widehat{\beta})$, otherwise one would get the contradiction $\operatorname{supp}(\widehat{\beta}) \subseteq \widehat{\Omega}_{\bullet}^{\operatorname{level}(\widehat{\beta})+1}$. In particular, this shows that

$$
\operatorname{level}(\widehat{\beta})=\min _{\substack{\widehat{T} \in \widehat{\mathcal{T}}_{\bullet}(\widehat{T} \subseteq \operatorname{supp}(\hat{\beta})}} \operatorname{level}(\widehat{T}) \quad \text { for all } \widehat{\beta} \in \widehat{\mathcal{H}}_{\bullet}
$$

Define the space of hierarchical splines in the parameter domain by $\widehat{\mathcal{Y}}_{\bullet}:=$ $\operatorname{span}\left(\widehat{\mathcal{H}}_{\bullet}\right)$. According to (3.14), each $\widehat{V}_{\bullet} \in \widehat{\mathcal{Y}}_{\bullet}$ is a $\widehat{\mathcal{T}}_{\bullet}$-piecewise tensor polynomial of 
degree $\left(p_{1}, \ldots, p_{d}\right)$. We define our ansatz space in the parameter domain as

$$
\begin{aligned}
\widehat{\mathcal{X}}_{\bullet} & :=\left\{\widehat{V}_{\bullet} \in \widehat{\mathcal{Y}}_{\bullet}:\left.\widehat{V}_{\bullet}\right|_{\partial \widehat{\Omega}}=0\right\} \subset \widehat{\mathcal{Y}}_{\bullet} \\
& \subset\left\{\widehat{v} \in W_{0}^{1, \infty}(\widehat{\Omega}):\left.\widehat{v}\right|_{\widehat{T}} \in C^{2}(\widehat{T}) \text { for all } \widehat{T} \in \widehat{\mathcal{T}}_{\bullet}\right\} .
\end{aligned}
$$

Note that this specifies the abstract setting of Sec. 2.3. For a more detailed introduction to hierarchical meshes and splines, we refer to, e.g. Refs. 45, 10] and 41.

\subsection{Basis of $\widehat{\boldsymbol{\mathcal { X }}}$.}

In this section, we characterize a basis of the hierarchical splines $\widehat{\mathcal{X}}_{\bullet}$ that vanish on the boundary. To this end, we first determine the restriction of the hierarchical basis $\widehat{\mathcal{H}}_{\bullet}$ to a facet of the boundary. It turns out that this restriction coincides with the set of $(d-1)$-dimensional hierarchical B-splines.

Proposition 3.1. Let $\widehat{\mathcal{T}}_{\bullet}$ be an arbitrary hierarchical mesh on the parameter domain $\widehat{\Omega}$. For $E=[0,1]^{I-1} \times\{e\} \times[0,1]^{d-I}$ with some $I \in\{1, \ldots, d\}$ and some $e \in\{0,1\}$, set $\left.\widehat{\mathcal{K}}^{0}\right|_{E}:=\left(\widehat{\mathcal{K}}_{1}^{0}, \ldots, \widehat{\mathcal{K}}_{I-1}^{0} \widehat{\mathcal{K}}_{I+1}^{0}, \ldots, \widehat{\mathcal{K}}_{d}^{0}\right)$, and $\left.\widehat{\Omega}_{\bullet}^{k}\right|_{E}:=$ $\left\{\left(s_{1}, \ldots, s_{I-1}, s_{I+1}, \ldots, s_{d}\right):\left(s_{1}, \ldots, s_{d}\right) \in \widehat{\Omega}_{\bullet}^{k} \cap E\right\}$ for $k \in \mathbb{N}_{0}$. Moreover, let $\left.\widehat{\mathcal{T}}_{\bullet}\right|_{E}$ be the corresponding hierarchical mesh and $\left.\widehat{\mathcal{H}}_{\bullet}\right|_{E}$ the corresponding hierarchical basis. Then, there holds* $\left.\widehat{\mathcal{H}}_{\bullet}\right|_{E}=\left\{\left.\widehat{\beta}\right|_{E}:\left.\widehat{\beta} \in \widehat{\mathcal{H}}_{\bullet} \wedge \widehat{\beta}\right|_{E} \neq 0\right\}$. Moreover, the restriction $\left.(\cdot)\right|_{E}:\left.\widehat{\mathcal{H}}_{\bullet} \rightarrow \widehat{\mathcal{H}}_{\bullet}\right|_{E}$ is essentially injective, i.e. for $\widehat{\beta}_{1}, \widehat{\beta}_{2} \in \widehat{\mathcal{H}}_{\bullet}$ with $\widehat{\beta}_{1} \neq \widehat{\beta}_{2}$ and $\left.\widehat{\beta}_{1}\right|_{E} \neq 0$, it follows that $\left.\widehat{\beta}_{1}\right|_{E} \neq\left.\widehat{\beta}_{2}\right|_{E}$.

Proof. We prove the assertion in two steps.

Step 1: Let $k \in \mathbb{N}_{0}$. We recall that the knot vectors $\widehat{\mathcal{K}}_{i}^{k}$ are $p_{i}$-open. In particular, this implies that the corresponding one-dimensional B-splines $\widehat{\mathcal{B}}_{i}^{k}$ are interpolatoric at the end points $e \in\{0,1\}$. This means that the first respectively, last B-spline in $\widehat{\mathcal{B}}_{i}^{k}$ (i.e. $B\left(\cdot \mid t_{i, 0}^{k}, \ldots, t_{i, 1+p_{i}}^{k}\right)$ respectively, $\left.B\left(\cdot \mid t_{i, N_{i}^{k}-1}^{k}, \ldots, t_{i, N_{i}^{k}+p_{i}}^{k}\right)\right)$ is equal to one at 0 respectively 1 and that all other B-splines of $\widehat{\mathcal{B}}_{i}^{k}$ vanish at these points; see, e.g. Lemma 2.1 of Ref. 38 .

Step 2: We consider arbitrary $d>1$. For $k \in \mathbb{N}_{0}$, let $\left.\widehat{\mathcal{B}}^{k}\right|_{E}$ be the set of tensor product B-splines induced by the reduced knots $\left.\widehat{\mathcal{K}}^{k}\right|_{E}$ which are defined analogously to $\left.\widehat{\mathcal{K}}^{0}\right|_{E}$. Since $\widehat{\mathcal{K}}_{j}^{k}$ is $p_{j}$-open, it holds that $\left.\widehat{\mathcal{B}}^{k}\right|_{E}=\left\{\left.\widehat{\beta}\right|_{E}:\left.\widehat{\beta} \in \widehat{\mathcal{B}}^{k} \wedge \widehat{\beta}\right|_{E} \neq 0\right\}$; see also Step 1. Then, the identity 3.12) shows

$$
\begin{aligned}
\left.\widehat{\mathcal{H}}_{\bullet}\right|_{E} & =\bigcup_{k \in \mathbb{N}_{0}}\left\{\left.\widehat{\beta}\right|_{E}:\left.\widehat{\beta} \in \widehat{\mathcal{B}}^{k} \wedge \widehat{\beta}\right|_{E} \neq 0 \wedge \operatorname{supp}\left(\left.\widehat{\beta}\right|_{E}\right)\right. \\
& \left.\left.\left.\subseteq \widehat{\Omega}_{\bullet}^{k}\right|_{E} \wedge \operatorname{supp}\left(\left.\widehat{\beta}\right|_{E}\right) \nsubseteq \widehat{\Omega}_{\bullet}^{k+1}\right|_{E}\right\} .
\end{aligned}
$$

* Actually, the set on left-hand side consists of functions defined on $[0,1]^{d-1}$, whereas the righthand side functions are defined on $E$. However, clearly these functions can be identified. 
Let $\widehat{\beta} \in \widehat{\mathcal{B}}^{k}$ for some $k \in \mathbb{N}_{0}$ with $\left.\widehat{\beta}\right|_{E} \neq 0$. We set $J:=0$ for $e=0$ respectively $J:=N_{I}^{k}-1$ for $e=1$. Since $\widehat{B}\left(e \mid t_{I, j_{I}}^{k}, \ldots, t_{I, j_{I}+p_{I}+1}^{k}\right)$ does not vanish only if $j_{I}=J$ (see Step 1), $\widehat{\beta}$ must be of the form

$$
\widehat{\beta}\left(s_{1}, \ldots, s_{d}\right)=\prod_{\substack{i=1 \\ i \neq I}}^{d} \widehat{B}\left(s_{i} \mid t_{i, j_{i}}^{k}, \ldots, t_{i, j_{i}+p_{i}+1}^{k}\right) \widehat{B}\left(s_{I} \mid t_{I, J}^{k}, \ldots, t_{I, J+p_{I}+1}^{k}\right),
$$

where the second factor is one if $s_{I}=e$ and satisfies $\operatorname{supp}\left(\widehat{B}\left(\cdot \mid t_{I, J}^{k}, \ldots, t_{I, J+p_{I}+1}^{k}\right)\right)=$ $\left[t_{I, J}^{k}, t_{I, J+p_{I}+1}^{k}\right]$. This shows that $\operatorname{supp}(\widehat{\beta})$ is the union of elements $\widehat{T} \in \widehat{\mathcal{T}}^{k}$ with nonempty intersection with $E$. Hence $\left.\operatorname{supp}\left(\left.\widehat{\beta}\right|_{E}\right) \subseteq \widehat{\Omega}_{\bullet}^{k}\right|_{E}$ is equivalent to $\operatorname{supp}(\widehat{\beta}) \subseteq$ $\widehat{\Omega}_{\bullet}^{k}$, and $\left.\operatorname{supp}\left(\left.\widehat{\beta}\right|_{E}\right) \nsubseteq \widehat{\Omega}_{\bullet}^{k+1}\right|_{E}$ is equivalent to $\operatorname{supp}(\widehat{\beta}) \nsubseteq \widehat{\Omega}_{\bullet}^{k+1}$. Therefore, (3.17) becomes

$$
\left.\widehat{\mathcal{H}}_{\bullet}\right|_{E}=\bigcup_{k \in \mathbb{N}_{0}}\left\{\left.\widehat{\beta}\right|_{E}:\left.\widehat{\beta} \in \widehat{\mathcal{B}}^{k} \wedge \widehat{\beta}\right|_{E} \neq 0 \wedge \operatorname{supp}(\widehat{\beta}) \subseteq \widehat{\Omega}_{\bullet}^{k} \wedge \operatorname{supp}(\widehat{\beta}) \not \subseteq \widehat{\Omega}_{\bullet}^{k+1}\right\} .
$$

Together with (3.12), this shows $\left.\widehat{\mathcal{H}}_{\bullet}\right|_{E}=\left\{\left.\widehat{\beta}\right|_{E}:\left.\widehat{\beta} \in \widehat{\mathcal{H}} \bullet \wedge \widehat{\beta}\right|_{E} \neq 0\right\}$. Finally, let

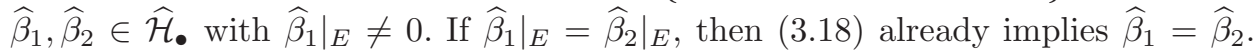
This concludes the proof.

Corollary 3.1. Let $\widehat{\mathcal{T}}_{\bullet}$ be an arbitrary hierarchical mesh on the parameter domain $\widehat{\Omega}$. Then, $\left\{\widehat{\beta} \in \widehat{\mathcal{H}}_{\bullet}:\left.\beta\right|_{\partial \widehat{\Omega}}=0\right\}$ is a basis of $\widehat{\mathcal{X}}_{\bullet}$.

Proof. Linear independence as well as $\left\{\widehat{\beta} \in \widehat{\mathcal{H}}_{\bullet}:\left.\widehat{\beta}\right|_{\partial \widehat{\Omega}}=0\right\} \subseteq \mathcal{X}_{\bullet}$ are obvious. To see $\mathcal{X}_{\bullet} \subseteq \operatorname{span}\left\{\widehat{\beta} \in \widehat{\mathcal{H}}_{\bullet}:\left.\widehat{\beta}\right|_{\partial \widehat{\Omega}}=0\right\}$, let $\widehat{V}_{\bullet} \in \widehat{\mathcal{X}}_{\bullet}$. Consider the unique representation $\widehat{V}_{\bullet}=\sum_{\widehat{\beta} \in \widehat{\mathcal{H}}_{\bullet}} c_{\widehat{\beta}} \widehat{\beta}$ with $c_{\widehat{\beta}} \in \mathbb{R}$. For arbitrary $\widehat{\beta} \in \widehat{\mathcal{H}}_{\bullet}$ with $\left.\widehat{\beta}\right|_{\partial \widehat{\Omega}} \neq 0$, we have to prove $c_{\widehat{\beta}}=0$, i.e. we have to show the implication

$$
\left.\sum_{\substack{\left.\widehat{\beta} \in \widehat{\mathcal{H}} \\ \widehat{\beta}\right|_{\partial \widehat{\Omega}} \neq 0}} c_{\widehat{\beta}} \widehat{\beta}\right|_{\partial \widehat{\Omega}}=0 \Rightarrow\left(\forall \widehat{\beta} \in \widehat{\mathcal{H}}_{\bullet} \text { with }\left.\widehat{\beta}\right|_{\partial \widehat{\Omega}} \neq 0 c_{\widehat{\beta}}=0\right) \text {. }
$$

Let $E=[0,1]^{I-1} \times\{e\} \times[0,1]^{d-I}$ with $I \in\{1, \ldots, d\}$ and $\left.\sum_{\left.\widehat{\beta} \in \widehat{\mathcal{H}}_{\bullet} \wedge \widehat{\beta}\right|_{E} \neq 0} c_{\widehat{\beta}} \widehat{\beta}\right|_{E}=$ 0 . According to Proposition 3.1 the family $\left(\left.\widehat{\beta}\right|_{E}:\left.\widehat{\beta} \in \widehat{\mathcal{H}} \bullet \wedge \widehat{\beta}\right|_{E} \neq 0\right)$ is linearly independent. Hence, $\widehat{c}_{\widehat{\beta}}=0$ for $\widehat{\beta} \in \widehat{\mathcal{H}}$ • with $\left.\widehat{\beta}\right|_{E} \neq 0$. Since $\partial \widehat{\Omega}$ is the union of such facets $E$, this concludes the proof.

\subsection{Admissible meshes in the parameter domain $\widehat{\mathbf{\Omega}}$}

Let $\widehat{\mathcal{T}}_{\bullet}$ be an arbitrary hierarchical mesh. We define the set of all neighbors of an element $\widehat{T} \in \widehat{\mathcal{T}}_{\bullet}$ as

$$
\mathcal{N}_{\bullet}(\widehat{T}):=\left\{\widehat{T}^{\prime} \in \widehat{\mathcal{T}}_{\bullet}: \exists \widehat{\beta} \in \widehat{\mathcal{H}} \bullet \widehat{T}, \widehat{T}^{\prime} \subseteq \operatorname{supp}(\widehat{\beta})\right\}
$$


According to (3.14), the condition $\widehat{T}, \widehat{T}^{\prime} \subseteq \operatorname{supp}(\widehat{\beta})$ is equivalent to $|\widehat{T} \cap \operatorname{supp}(\widehat{\beta})| \neq$ $0 \neq\left|\widehat{T}^{\prime} \cap \operatorname{supp}(\widehat{\beta})\right|$. We call $\widehat{\mathcal{T}}_{\bullet}$ admissible if

$$
\left|\operatorname{level}(\widehat{T})-\operatorname{level}\left(\widehat{T}^{\prime}\right)\right| \leq 1 \quad \text { for all } \widehat{T}, \widehat{T}^{\prime} \in \widehat{\mathcal{T}}_{\bullet} \text { with } \widehat{T}^{\prime} \in \mathcal{N}_{\bullet}(\widehat{T}) .
$$

Let $\widehat{\mathbb{T}}$ be the set of all admissible hierarchical meshes in the parameter domain. Clearly, $\widehat{\mathcal{T}}^{k} \in \widehat{\mathbb{T}}$ for all $k \in \mathbb{N}_{0}$. Moreover, admissible meshes satisfy the following interesting properties which are also important for an efficient implementation of IGAFEM with hierarchical splines.

Proposition 3.2. Let $\widehat{\mathcal{T}}_{\bullet} \in \widehat{\mathbb{T}}$. Then, the support of any basis function $\widehat{\beta} \in \widehat{\mathcal{H}}$. is the union of at most $2^{d}(p+1)^{d}$ elements $\widehat{T}^{\prime} \in \widehat{\mathcal{T}}_{\bullet}$. Moreover, for any $\widehat{T} \in \widehat{\mathcal{T}}_{\bullet}$, there are at most $2(p+1)^{d}$ basis functions $\widehat{\beta}^{\prime} \in \widehat{\mathcal{H}}_{\bullet}$ that have support on $\widehat{T}$, i.e. $\left|\operatorname{supp}\left(\widehat{\beta}^{\prime}\right) \cap \widehat{T}\right|>0$.

Proof. We abbreviate $k:=\operatorname{level}(\widehat{\beta})$. By $(\underline{3.15})$, there exists $\widehat{T}^{\prime \prime} \subseteq \operatorname{supp}(\widehat{\beta})$ with level $\left(\widehat{T}^{\prime \prime}\right)=k$. Admissibility of $\widehat{\mathcal{T}}_{\bullet}$ together with 3.14 shows that level $\left(\widehat{T}^{\prime}\right) \in$ $\{k, k+1\}$ for all $\widehat{T}^{\prime} \in \widehat{\mathcal{T}}_{\bullet}$ with $\widehat{T}^{\prime} \subseteq \operatorname{supp}(\widehat{\beta})$. Since $\widehat{\beta}$ is an element of $\widehat{\mathcal{B}}^{k}$, its support is the union of at most $2^{d}(p+1)^{d}$ elements in $\widehat{\mathcal{T}}^{k+1}$. This proves the first assertion. For $\widehat{\beta}^{\prime} \in \widehat{\mathcal{H}}_{\bullet}$ and $\widehat{T} \in \widehat{\mathcal{T}}_{\bullet}$ with $\left|\operatorname{supp}\left(\widehat{\beta}^{\prime}\right) \cap \widehat{T}\right|>0$, the characterization (3.14) proves $\widehat{T} \subseteq \operatorname{supp}\left(\widehat{\beta}^{\prime}\right)$. Hence, (3.15) together with admissibility of $\widehat{\mathbb{T}}$ proves that $\operatorname{level}\left(\widehat{\beta}^{\prime}\right)=\widetilde{k}:=\operatorname{level}(\widehat{T})$ or $\operatorname{level}\left(\widehat{\beta}^{\prime}\right)=\widetilde{k}-1$. With $\widehat{\mathcal{B}}^{-1}:=\widehat{\mathcal{B}}^{0}$, there are at most $(p+1)^{d}$ basis functions in $\widehat{\mathcal{B}}^{\widetilde{k}-1}$ and $(p+1)^{d}$ basis functions in $\widehat{\mathcal{B}}^{\widetilde{k}}$ that have support on the element $\widehat{T}$. This concludes the proof.

Remark 3.1. Since the support of any $\widehat{\beta} \in \widehat{\mathcal{H}}_{\bullet}$ is connected, Proposition 3.2 particularly shows that $\widehat{T}^{\prime} \subseteq \operatorname{supp}(\widehat{\beta})$ for an element $\widehat{T}^{\prime} \in \widehat{\mathcal{T}}_{\bullet}$ implies that $\operatorname{supp}(\widehat{\beta}) \subseteq$ $\pi_{\bullet}^{2(p+1)}\left(\widehat{T}^{\prime}\right)$. Moreover, we recall that $\widehat{T}^{\prime} \subseteq \operatorname{supp}(\widehat{\beta})$ is equivalent to $\left|\widehat{T}^{\prime} \cap \operatorname{supp}(\widehat{\beta})\right|>$ $0 ;$ see (3.14).

\subsection{Refinement in the parameter domain $\widehat{\boldsymbol{\Omega}}$}

We define the initial mesh $\widehat{\mathcal{T}}_{0}:=\widehat{\mathcal{T}}^{0}$. Note that $\widehat{\mathcal{T}}_{0}$ is a hierarchical mesh with $\widehat{\Omega}_{0}^{k}=\emptyset$ for all $k>0$. We say that a hierarchical mesh $\widehat{\mathcal{T}}_{\circ}$ is finer than another hierarchical mesh $\widehat{\mathcal{T}}_{\bullet}$ if $\widehat{\Omega}_{\bullet}^{k} \subseteq \widehat{\Omega}_{\circ}^{k}$ for all $k \in \mathbb{N}_{0}$. This just means that $\widehat{\mathcal{T}}_{\circ}$ is obtained from $\widehat{\mathcal{T}}_{\bullet}$ by iterative dyadic bisections of the elements in $\widehat{\mathcal{T}}_{\bullet}$. To bisect an element $\widehat{T} \in \widehat{\mathcal{T}}_{\bullet}$, one just has to add it to the set $\widehat{\Omega}_{\bullet}^{\operatorname{level}(\widehat{T})+1}$, see (3.24) below. In this case, the corresponding spaces are nested, i.e.

$$
\widehat{\mathcal{Y}}_{\bullet} \subseteq \widehat{\mathcal{Y}}_{\circ} \text { and } \widehat{\mathcal{X}}_{\bullet} \subseteq \widehat{\mathcal{X}}_{\circ}
$$

For a proof, see, e.g. Corollary 2 of Ref. 41. In particular, this implies

$$
\widehat{\mathcal{Y}}^{0} \subseteq \widehat{\mathcal{Y}}_{\bullet} \subseteq \widehat{\mathcal{Y}}^{M \bullet-1}
$$


Next, we present a concrete refinement algorithm to specify the setting of Sec. 2.2, To this end, we first define for $\widehat{T} \in \widehat{\mathcal{T}}_{\bullet} \in \mathbb{T}$ the set of its bad neighbors

$$
\mathcal{N}_{\bullet}^{\text {bad }}(\widehat{T}):=\left\{\widehat{T}^{\prime} \in \mathcal{N}_{\bullet}(\widehat{T}): \operatorname{level}\left(\widehat{T}^{\prime}\right)=\operatorname{level}(\widehat{T})-1\right\} \text {. }
$$

Algorithm 3.1. Input: Hierarchical mesh $\widehat{\mathcal{T}}_{\bullet}$, marked elements $\widehat{\mathcal{M}}_{\bullet}=: \widehat{\mathcal{M}}_{\bullet}^{(0)} \subseteq \widehat{\mathcal{T}}_{\bullet}$.

(i) Iterate the following Steps (a)-(b) for $i=0,1,2, \ldots$ until $\widehat{\mathcal{U}}_{\bullet}^{(i)}=\emptyset$ :

(a) Define $\widehat{\mathcal{U}}_{\bullet}^{(i)}:=\bigcup_{\widehat{T} \in \widehat{\mathcal{M}}_{\bullet}^{(i)}}\left\{\widehat{T}^{\prime} \in \widehat{\mathcal{T}}_{\bullet} \backslash \widehat{\mathcal{M}}_{\bullet}^{(i)}: \widehat{T}^{\prime} \in \mathcal{N}_{\bullet}^{\text {bad }}(T)\right\}$.

(b) Define $\widehat{\mathcal{M}}_{\bullet}^{(i+1)}:=\widehat{\mathcal{M}}_{\bullet}^{(i)} \cup \widehat{\mathcal{U}}_{\bullet}^{(i)}$.

(ii) Dyadically bisect all $\widehat{T} \in \widehat{\mathcal{M}}_{\bullet}^{(i)}$ by adding $\widehat{T}$ to the set $\widehat{\Omega}_{\bullet}^{\operatorname{level}(\widehat{T})+1}$ and obtain a finer hierarchical mesh $\widehat{\mathcal{T}}_{\circ}=\operatorname{refine}\left(\widehat{\mathcal{T}}_{\bullet}, \widehat{\mathcal{M}}_{\bullet}\right)$, where

$$
\widehat{\Omega}_{\circ}^{k}=\widehat{\Omega}_{\bullet}^{k} \cup \bigcup\left\{\widehat{T} \in \widehat{\mathcal{M}}_{\bullet}^{(i)}: \operatorname{level}(\widehat{T})=k-1\right\} \quad \text { for all } k \in \mathbb{N} .
$$

Output: Refined mesh $\widehat{\mathcal{T}}_{\circ}:=\operatorname{refine}\left(\widehat{\mathcal{T}}_{\bullet}, \widehat{\mathcal{M}}_{\bullet}\right)$.

Clearly, refine $\left(\widehat{\mathcal{T}}_{\bullet}, \widehat{\mathcal{M}}_{\bullet}\right)$ is finer than $\widehat{\mathcal{T}}_{\bullet}$. For any hierarchical mesh $\widehat{\mathcal{T}}_{\bullet}$, we define refine $\left(\widehat{\mathcal{T}}_{\bullet}\right)$ as the set of all hierarchical meshes $\widehat{\mathcal{T}}_{0}$ such that there exist hierarchical meshes $\widehat{\mathcal{T}}_{(0)}, \ldots, \widehat{\mathcal{T}}_{(J)}$ and marked elements $\widehat{\mathcal{M}}_{(0)}, \ldots, \widehat{\mathcal{M}}_{(J-1)}$ with $\widehat{\mathcal{T}}_{\circ}=\widehat{\mathcal{T}}_{(J)}=$ $\operatorname{refine}\left(\widehat{\mathcal{T}}_{(J-1)}, \widehat{\mathcal{M}}_{(J-1)}\right), \ldots, \widehat{\mathcal{T}}_{(1)}=\operatorname{refine}\left(\widehat{\mathcal{T}}_{(0)}, \widehat{\mathcal{M}}_{(0)}\right)$, and $\widehat{\mathcal{T}}_{(0)}=\widehat{\mathcal{T}}_{\bullet}$. Here, we formally allow $J=0$, i.e. $\mathcal{T}_{\bullet} \in \operatorname{refine}\left(\mathcal{T}_{\bullet}\right)$. Proposition 5.1 below will show that $\widehat{\mathbb{T}}=\operatorname{refine}\left(\widehat{\mathcal{T}}_{0}\right)$, i.e. starting from $\widehat{\mathcal{T}}_{0}=\widehat{\mathcal{T}}^{0}$, all admissible meshes $\widehat{\mathcal{T}}_{\bullet}$ can be generated by iterative refinement via Algorithm 3.1.

Remark 3.2. References 11 and 13 studied a related refinement strategy, where $\mathcal{N}_{\bullet}(\widehat{T})$ of 3.19 and $\mathcal{N}_{\bullet}^{\operatorname{bad}}(\widehat{T})$ from $(3.23)$ are replaced by:

$$
\begin{aligned}
\widetilde{\mathcal{N}}_{\bullet}(\widehat{T}):= & \left\{\widehat{T}^{\prime} \in \widehat{\mathcal{T}}_{\bullet}: \exists \widehat{\beta} \in \widehat{\mathcal{B}}^{\operatorname{level}(\widehat{T})}\right. \text { with } \\
& \left.|\widehat{T} \cap \operatorname{supp}(\widehat{\beta})| \neq 0 \neq\left|\widehat{T}^{\prime} \cap \operatorname{supp}(\widehat{\beta})\right|\right\}, \\
\tilde{\mathcal{N}}_{\bullet}^{\operatorname{bad}}(\widehat{T}):= & \left\{\widehat{T}^{\prime} \in \widetilde{\mathcal{N}}_{\bullet}: \operatorname{level}\left(\widehat{T}^{\prime}\right)=\operatorname{level}(\widehat{T})-1\right\} .
\end{aligned}
$$

There, the refinement strategy was designed for truncated hierarchical B-splines; see Sec. [5.9. Compared to the hierarchical B-splines $\widehat{\mathcal{H}}_{\bullet}$, those have generically a smaller, but also more complicated and not necessarily connected support. Corollary 17 of Ref. 11 shows that the generated meshes are strictly admissible in the sense of Refs. 11 and 13, i.e. for all $k \in \mathbb{N}$, it holds that

$$
\widehat{\Omega}_{\bullet}^{k} \subseteq \bigcup\left\{\widehat{T} \in \widehat{\mathcal{T}}^{k-1}: \forall \widehat{\beta} \in \widehat{\mathcal{B}}^{k-1}\left(\widehat{T} \subseteq \operatorname{supp}(\widehat{\beta}) \Rightarrow \operatorname{supp}(\widehat{\beta}) \subseteq \widehat{\Omega}_{\bullet}^{k-1}\right)\right\} .
$$

This definition actually goes back to Appendix A of Ref. 27. According to Sec. 2.4 of Ref. 11, strictly admissible meshes satisfy a similar version of Proposition 3.2 for truncated hierarchical B-splines. However, the example from Fig. 2 shows that the proposition fails for hierarchical B-splines and the refinement 

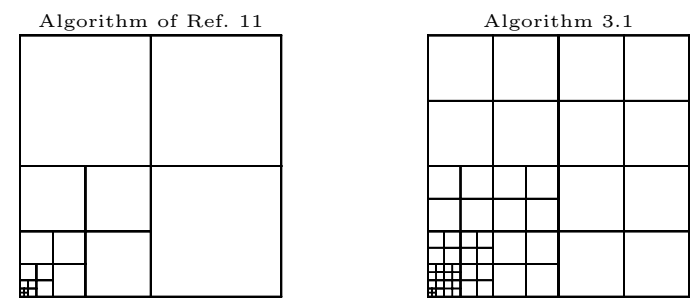

Fig. 2. An initial mesh $\widehat{\mathcal{T}}_{0}$ with only one element $[0,1]^{2}$ is locally refined in the lower left corner using the refinement of Ref. 11 (left) respectively the refinement of Algorithm 3.1 (right); see Remark 3.2 Consider the lowest-order case $\left(p_{1}, p_{2}\right)=(1,1)$. By repetitive refinement via Ref. 11, the number of elements in the support of the hierarchical B-spline $\widehat{B}\left(s_{1} \mid 0,1 / 2,1\right) \widehat{B}\left(s_{2} \mid 0,1 / 2,1\right)$ grows to infinity. Moreover, the number of hierarchical B-splines with support on the element in the lower left corner grows to infinity. This is not the case if one uses Algorithm 3.1, see also Proposition 3.2

strategy from Ref. 11. In particular, strictly admissible meshes are not necessarily admissible in the sense of Sec. 3.4

\subsection{Hierarchical meshes and splines in the physical domain $\Omega$}

To transform the definitions in the parameter domain to the physical domain, we assume that we are given a bi-Lipschitz continuous piecewise $C^{2}$-parametrization:

$$
\begin{gathered}
\gamma: \bar{\Omega} \rightarrow \bar{\Omega} \quad \text { with } \quad \gamma \in W^{1, \infty}(\widehat{\Omega}) \cap C^{2}\left(\widehat{\mathcal{T}}_{0}\right) \quad \text { and } \\
\gamma^{-1} \in W^{1, \infty}(\Omega) \cap C^{2}\left(\mathcal{T}_{0}\right),
\end{gathered}
$$

where $C^{2}\left(\mathcal{T}_{0}\right):=\left\{v: \bar{\Omega} \rightarrow \mathbb{R}:\left.v\right|_{T} \in C^{2}(T)\right.$ for all $\left.T \in \mathcal{T}_{0}\right\}$. Consequently, there exists $C_{\gamma}>0$ such that for all $i, j, k \in\{1, \ldots, d\}$ :

$$
\begin{gathered}
\left\|\frac{\partial}{\partial t_{j}} \gamma_{i}\right\|_{L^{\infty}(\widehat{\Omega})} \leq C_{\gamma}, \quad\left\|\frac{\partial}{\partial x_{j}}\left(\gamma^{-1}\right)_{i}\right\|_{L^{\infty}(\Omega)} \leq C_{\gamma}, \\
\left\|\frac{\partial^{2}}{\partial t_{j} \partial t_{k}} \gamma_{i}\right\|_{L^{\infty}(\widehat{\Omega})} \leq C_{\gamma}, \quad\left\|\frac{\partial^{2}}{\partial x_{j} \partial x_{k}}\left(\gamma^{-1}\right)_{i}\right\|_{L^{\infty}(\Omega)} \leq C_{\gamma},
\end{gathered}
$$

where $\gamma_{i}$ respectively, $\left(\gamma^{-1}\right)_{i}$ denotes the $i$ th component of $\gamma$ respectively, $\gamma^{-1}$. All previous definitions can now also be made in the physical domain, just by pulling them from the parameter domain via the diffeomorphism $\gamma$. For these definitions, we drop the symbol $\widehat{.}$. If $\widehat{\mathcal{T}} \bullet \in \widehat{\mathbb{T}}$, we define the corresponding mesh in the physical domain as $\mathcal{T}_{\bullet}:=\left\{\gamma(\widehat{T}): \widehat{T} \in \widehat{\mathcal{T}}_{\bullet}\right\}$. In particular, we have $\mathcal{T}_{0}=\left\{\gamma(\widehat{T}): \widehat{T} \in \widehat{\mathcal{T}}_{0}\right\}$. Moreover, let $\mathbb{T}:=\left\{\mathcal{T}_{\bullet}: \widehat{\mathcal{T}}_{\bullet} \in \widehat{\mathbb{T}}\right\}$ be the set of admissible meshes in the physical domain. If now $\mathcal{M}_{\bullet} \subseteq \mathcal{T}_{\bullet}$ with $\mathcal{T}_{\bullet} \in \mathbb{T}$, we abbreviate $\widehat{\mathcal{M}}_{\bullet}:=\left\{\gamma^{-1}(T): T \in \mathcal{M}_{\bullet}\right\}$ and define refine $\left(\mathcal{T}_{\bullet}, \mathcal{M}_{\bullet}\right):=\left\{\gamma(\widehat{T}): \widehat{T} \in \operatorname{refine}\left(\widehat{\mathcal{T}}_{\bullet}, \widehat{\mathcal{M}}_{\bullet}\right)\right\}$. For $\mathcal{T}_{\bullet} \in \mathbb{T}$, let $\mathcal{X}_{\bullet}:=$ $\left\{\widehat{V}_{\bullet} \circ \gamma^{-1}: \widehat{V}_{\bullet} \in \widehat{\mathcal{X}}_{\bullet}\right\}$ be the corresponding hierarchical spline space. By regularity of $\gamma$, we especially obtain

$$
\mathcal{X}_{\bullet} \subset\left\{v \in H_{0}^{1}(\Omega):\left.v\right|_{T} \in H^{2}(T) \text { for all } T \in \mathcal{T}_{\bullet}\right\} .
$$




\subsection{Main result}

Before we come to the main result of this work, we fix polynomial orders $\left(q_{1}, \ldots, q_{d}\right)$ and define for $\mathcal{T}_{\bullet} \in \mathbb{T}$ the space of transformed polynomials

$$
\mathcal{P}(\Omega):=\left\{\widehat{V} \circ \gamma: \widehat{V} \text { is a tensor polynomial of order }\left(q_{1}, \ldots, q_{d}\right)\right\} .
$$

Remark 3.3. In order to obtain higher-order oscillations, the natural choice of the polynomial orders is $q_{i} \geq 2 p_{i}-1$; see, e.g. Sec. 3.1 of Ref. 36 If $\mathcal{X} \bullet \subset C^{1}(\bar{\Omega})$, it is sufficient to choose $q_{i} \geq 2 p_{i}-2$; see Remark 2.2.

Altogether, we have specified the abstract framework of Sec. 2 to hierarchical meshes and splines. The following theorem is the main result of the present work. It shows that all assumptions of Theorem 2.1] are satisfied for the present IGAFEM approach. The proof is given in Sec. 5.

Theorem 3.1. Hierarchical splines on admissible meshes satisfy the abstract assumptions (M1)-(M3), (R1)-(R5) and (S1)-(S6) from Sec. 2, where the constants depend only on $d, C_{\gamma}, \widehat{\mathcal{T}}_{0}$, and $\left(p_{1}, \ldots, p_{d}\right)$. Moreover, the piecewise polynomials $\mathcal{P}(\Omega)$ from (3.30) on admissible meshes satisfy the abstract assumptions (O1)-(O4), where the constants depend only on $d, C_{\gamma}, \widehat{\mathcal{T}}_{0}$, and $\left(q_{1}, \ldots, q_{d}\right)$. By Theorem 2.1, this implies reliability (2.14) as well as efficiency (2.15) of the error estimator, linear convergence (2.16), and quasi-optimal convergence rates (2.17) for the adaptive strategy from Algorithm 2.1 .

Remark 3.4. If one applies continuous piecewise polynomials of degree $p$ on a triangulation of some polygonal respectively, polyhedral domain $\Omega$ as ansatz space, Ref. 25] proves that $\|u\|_{\mathbb{B}_{p / d}}<\infty$. The proof requires that $u$ allows for a certain decomposition and that the oscillations are of higher order; see Remark 3.3. In our case, $\|u\|_{\mathbb{A}_{s}} \simeq\|u\|_{\mathbb{B}_{s}}($ see (2.18) $)$ depends besides the polynomial degrees $\left(p_{1}, \ldots, p_{d}\right)$ also on the (piecewise) smoothness of the parametrization $\gamma$. In practice, $\gamma$ is usually piecewise $C^{\infty}$; see Sec. 6. Given this additional regularity of $\gamma$, one might expect that the result of Ref. 25] can be generalized such that $\|u\|_{\mathbb{A}_{s}},\|u\|_{\mathbb{B}_{s}}<\infty$ for $s=$ $\min _{i=1, \ldots, d} p_{i} / d$. However, the proof goes beyond the scope of this work and is left for future research.

\subsection{Generalization to rational hierarchical splines}

One can easily verify that all theoretical results of this work are still valid if one replaces the ansatz space $\mathcal{X}$. by rational hierarchical splines, i.e. by the set

$$
\mathcal{X}_{\bullet}^{W_{0}}:=\left\{\frac{V_{\bullet}}{W_{0}}: V_{\bullet} \in \mathcal{X}_{\bullet}\right\},
$$

where $W_{0}$ is a fixed positive weight function in the initial ansatz space $\mathcal{X}_{0}$. In this case, the corresponding basis consists of NURBS instead of B-splines. Indeed, the mesh properties (M1)-(M3) as well as the refinement properties (R1)-(R5) from 
Sec. 2 are independent of the discrete spaces. To verify the validity of Theorem 3.1 in the NURBS setting, it thus only remains to verify the properties (S1)-(S6) for the NURBS finite element spaces. The inverse estimate (S1) follows similarly as in Sec. [5.6 since we only consider a fixed and thus uniformly bounded weight function $W_{0} \in \mathcal{X}_{0}$. The properties (S2)-(S3) depend only on the numerator of the NURBS functions and thus transfer. To see (S4)-(S6), one can proceed as in Sec. 5.10, where the corresponding Scott-Zhang-type operator $J_{\bullet}^{W_{0}}: L^{2}(\Omega) \rightarrow \mathcal{X}_{\bullet}^{W_{0}}$ now reads

$$
J_{\bullet}^{W_{0}} v:=\frac{J_{\bullet}\left(v W_{0}\right)}{W_{0}} \quad \text { for all } v \in L^{2}(\Omega) .
$$

With this definition, Lemma 5.6 holds accordingly, and (S4)-(S6) are proved as in Sec. 5.10 .

\section{Sketch of Proof of Theorem 2.1}

In the following subsections, we sketch the proof of Theorem 2.1, where we build upon the analysis of Ref. 14. Recall the residual a posteriori error estimator $\eta_{\bullet}$ from Sec. 2.4

\subsection{Discrete reliability}

Under the assumptions (M2)-(M3), and (S2)-(S6), we show that there exists $C_{\text {drel }}, C_{\text {ref }} \geq 1$ such that for all $\mathcal{T}_{\bullet} \in \mathbb{T}$ and all $\mathcal{T}_{\circ} \in \operatorname{refine}\left(\mathcal{T}_{\bullet}\right)$, the subset $\mathcal{R}_{\bullet}:=\Pi_{\bullet}^{\text {loc }}\left(\mathcal{T}_{\bullet} \backslash \mathcal{T}_{\circ}\right) \subseteq \mathcal{T}_{\bullet}$ satisfies

$$
\left\|U_{\circ}-U_{\bullet}\right\|_{H^{1}(\Omega)} \leq C_{\text {drel }} \eta_{\bullet}\left(\mathcal{R}_{\bullet}\right), \quad \mathcal{T}_{\bullet} \backslash \mathcal{T}_{\circ} \subseteq \mathcal{R}_{\bullet \circ}, \quad \text { and } \quad \# \mathcal{R}_{\bullet \circ} \leq C_{\text {ref }} \#\left(\mathcal{T}_{\bullet} \backslash \mathcal{T}_{\circ}\right) \text {. }
$$

The last two properties are obvious with $C_{\text {ref }}=C_{\text {patch }} k_{\text {loc }}$ by validity of (M2) and (S3). For the first property, we argue as in Theorem 4.1 of Ref. 42 . Ellipticity (1.4), $e_{\circ}:=U_{\circ}-U_{\bullet} \in \mathcal{X}_{\circ}$ (which follows from (S2)), and Galerkin orthogonality (2.6) with $V_{\bullet}:=J_{\bullet} e_{\circ} \in \mathcal{X}_{\bullet}$ prove

$$
\begin{aligned}
\left\|U_{\circ}-U_{\bullet}\right\|_{H^{1}(\Omega)}^{2} \lesssim\left\langle e_{\circ}, e_{\circ}\right\rangle_{\mathcal{L}} & =\left\langle e_{\circ},\left(1-J_{\bullet}\right) e_{\circ}\right\rangle_{\mathcal{L}} \\
& =\int_{\Omega} f\left(1-J_{\bullet}\right) e_{\circ} d x-\left\langle U_{\bullet},\left(1-J_{\bullet}\right) e_{\circ}\right\rangle_{\mathcal{L}} .
\end{aligned}
$$

We split $\Omega$ into elements $T \in \mathcal{T}_{\bullet}$ and apply elementwise integration by parts, where we denote the outer normal vector by $\nu$. With $\left.U_{\bullet}\right|_{T} \in H^{2}(T)$, this leads to

$$
\begin{aligned}
= & \sum_{T \in \mathcal{T}_{\bullet}}\left(\int_{T} f\left(1-J_{\bullet}\right) e_{\circ} d x-\int_{T}\left(-\operatorname{div}\left(\mathbf{A} \nabla U_{\bullet}\right)+\mathbf{b} \nabla U_{\bullet}+c U_{\bullet}\right)\left(1-J_{\bullet}\right) e_{\circ} d x\right. \\
& \left.+\int_{\partial T}\left(\mathbf{A} \nabla U_{\bullet} \cdot \nu\right)\left(1-J_{\bullet}\right) e_{\circ} d s\right) .
\end{aligned}
$$

The properties (S3)-(S4) immediately prove for any $V_{\circ} \in \mathcal{X}_{\circ}$ :

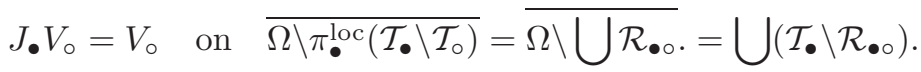


Hence, the sum in (4.1) reduces from $T \in \mathcal{T}_{\bullet}$ to $T \in \mathcal{R}_{\bullet}$. Recall that $\left(1-J_{\bullet}\right) e_{\circ} \in$ $\mathcal{X}_{\circ} \subset H_{0}^{1}(\Omega)$ with $\left(1-J_{\bullet}\right) e_{\circ}=0$ on $\partial\left(\bigcup \mathcal{R}_{\bullet \circ}\right)$. We define the set of facets $\mathcal{E}_{\bullet \circ}:=$ $\left\{T_{1} \cap T_{2}: T_{1}, T_{2} \in \mathcal{R} \bullet\right.$ with $\left.T_{1} \neq T_{2} \wedge\left|T_{1} \cap T_{2}\right|>0\right\}$, where $|\cdot|$ denotes the $(d-1)$ dimensional measure. Almost all $x \in \bigcup \mathcal{E}_{\bullet}$ belong to precisely two elements with opposite normal vectors. Hence,

$$
\begin{aligned}
\sum_{T \in \mathcal{R}_{\bullet}} \int_{\partial T}\left(\mathbf{A} \nabla U_{\bullet} \cdot \nu\right)\left(1-J_{\bullet}\right) e_{\circ} d s & =\sum_{T \in \mathcal{R}_{\bullet} \circ} \int_{\partial T \cap \Omega}\left(\mathbf{A} \nabla U_{\bullet} \cdot \nu\right)\left(1-J_{\bullet}\right) e_{\circ} d s \\
& \leq \sum_{E \in \mathcal{E}_{\bullet}} \int_{E}\left|\left[\mathbf{A} \nabla U_{\bullet} \cdot \nu\right]\left(1-J_{\bullet}\right) e_{\circ}\right| d s \\
& =\frac{1}{2} \sum_{T \in \mathcal{R} \bullet} \int_{\partial T \cap \Omega}\left|\left[\mathbf{A} \nabla U_{\bullet} \cdot \nu\right]\left(1-J_{\bullet}\right) e_{\circ}\right| d s
\end{aligned}
$$

Altogether, we have derived

$$
\begin{aligned}
\| U_{\circ} & -U_{\bullet} \|_{H^{1}(\Omega)}^{2} \\
& \lesssim \sum_{T \in \mathcal{R} \bullet \circ}\left(\int_{T}\left(f-\mathcal{L} U_{\bullet}\right)\left(1-J_{\bullet}\right) e_{\circ} d x+\int_{\partial T \cap \Omega}\left|\left[\mathbf{A} \nabla U_{\bullet} \cdot \nu\right]\left(1-J_{\bullet}\right) e_{\circ}\right| d s\right) \\
\leq & \sum_{T \in \mathcal{R} \bullet}\left(|T|^{1 / d}\left\|f-\mathcal{L} U_{\bullet}\right\|_{L^{2}(T)}|T|^{-1 / d}\left\|\left(1-J_{\bullet}\right) e_{\circ}\right\|_{L^{2}(T)}\right. \\
& \left.+|T|^{1 /(2 d)}\left\|\left[\mathbf{A} \nabla U_{\bullet} \cdot \nu\right]\right\|_{L^{2}(\partial T \cap \Omega)}|T|^{-1 /(2 d)}\left\|\left(1-J_{\bullet}\right) e_{\circ}\right\|_{L^{2}(\partial T \cap \Omega)}\right)
\end{aligned}
$$

We abbreviate $\pi_{\bullet}^{\max }:=\pi_{\bullet}^{\max \left(k_{\mathrm{app}}, k_{\mathrm{grad}}\right)}$. By (M3), (S5) and (S6), we have $|T|^{-1 / d}\left\|\left(1-J_{\bullet}\right) e_{\circ}\right\|_{L^{2}(T)}+|T|^{-1 /(2 d)}\left\|\left(1-J_{\bullet}\right) e_{\circ}\right\|_{L^{2}(\partial T \cap \Omega)} \lesssim\left\|U_{\circ}-U_{\bullet}\right\|_{H^{1}\left(\pi_{\bullet}^{\max }(T)\right)}$.

Plugging this into (4.2) and using the Cauchy-Schwarz inequality, we obtain

$$
\left\|U_{\circ}-U_{\bullet}\right\|_{H^{1}(\Omega)}^{2} \lesssim\left(\sum_{T \in \mathcal{R} \bullet \circ} \eta_{\bullet}(T)^{2}\right)^{1 / 2}\left(\sum_{T \in \mathcal{R} \bullet \circ}\left\|U_{\circ}-U_{\bullet}\right\|_{H^{1}\left(\pi_{\bullet}^{\max }(T)\right)}^{2}\right)^{1 / 2} .
$$

With (M2), the second factor is controlled by $\left\|U_{\circ}-U_{\bullet}\right\|_{H^{1}(\Omega)}$. This concludes the current section, and $C_{\text {drel }}$ depends only on $C_{\text {ell }}$, (M2)-(M3) and (S2)-(S6).

\subsection{Reliability (2.14)}

Note that osc $\bullet \lesssim \eta_{\bullet}$ follows immediately from their definitions (2.9)-(2.10) . If one replaces $U_{\circ} \in \mathcal{X}_{\circ}$ by the exact solution $u \in H_{0}^{1}(\Omega)$ and $\mathcal{R}_{\bullet}$ by $\mathcal{T}_{\bullet}$, reliability (2.14) follows along the lines of Sec. 4.1, but now, (S2)-(S4) are not needed for the proof. 


\subsection{Efficiency (2.15)}

As in Theorem 7 of Ref. 36, the assumptions (M1)-(M4) and (O1)-(O4) imply that

$$
\eta_{\bullet} \lesssim\left\|u-U_{\bullet}\right\|_{H^{1}(\Omega)}+\operatorname{osc}_{\bullet}\left(U_{\bullet}\right) .
$$

As in Proposition 3.3 of Ref. 15, the assumptions (M1)-(M3) and (S1) imply that

$$
\operatorname{osc}\left(U_{\bullet}\right) \lesssim \operatorname{osc}\left(V_{\bullet}\right)+\left\|U_{\bullet}-V_{\bullet}\right\|_{H^{1}(\Omega)} \quad \text { for all } V_{\bullet} \in \mathcal{X}_{\bullet} .
$$

The Céa lemma (2.7) and (4.4) show that

$$
\begin{aligned}
\left\|u-U_{\bullet}\right\|_{H^{1}(\Omega)}+\operatorname{Osc} \bullet(U \bullet) & \stackrel{\sqrt{4.4)}}{\lesssim}\left\|u-U_{\bullet}\right\|_{H^{1}(\Omega)}+\operatorname{osc}_{\bullet}\left(V_{\bullet}\right)+\left\|u-V_{\bullet}\right\|_{H^{1}(\Omega)} \\
& \stackrel{(2.7)}{\lesssim}\left\|u-V_{\bullet}\right\|_{H^{1}(\Omega)}+\operatorname{osc}_{\bullet}\left(V_{\bullet}\right) \quad \text { for all } V_{\bullet} \in \mathcal{X}_{\bullet} .
\end{aligned}
$$

This proves $\left\|u-U_{\bullet}\right\|_{H^{1}(\Omega)}+\operatorname{osc}_{\bullet}\left(U_{\bullet}\right) \simeq \inf _{V_{\bullet} \in \mathcal{X}}\left(\left\|u-V_{\bullet}\right\|_{H^{1}(\Omega)}+\operatorname{osc}_{\bullet}\left(V_{\bullet}\right)\right)$. Combining these observations, we conclude (2.15), where $C_{\text {eff }}$ depends only on $C_{\text {ell }}$, (M1)-(M4), (S1) and (O1)-(O4).

\subsection{Stability on non-refined elements}

As in Corollary 3.4 of Ref. 15, the assumptions (M1)-(M3) and (S1) imply the existence of $C_{\text {stab }} \geq 1$ such that for all $\mathcal{T}_{\bullet} \in \mathbb{T}$, all $\mathcal{T}_{\circ} \in \operatorname{refine}\left(\mathcal{T}_{\bullet}\right)$, and all subsets $\mathcal{S} \subseteq \mathcal{T}_{\bullet} \cap \mathcal{T}_{\circ}$ of non-refined elements, it holds that

$$
\left|\eta_{\bullet}(\mathcal{S})-\eta_{\circ}(\mathcal{S})\right| \leq C_{\text {stab }}\left\|U_{\bullet}-U_{\circ}\right\|_{H^{1}(\Omega)} .
$$

The constant $C_{\text {stab }}$ depends only on (M1)-(M3), (S1), as well as on $\|\mathbf{A}\|_{W^{1, \infty}(\Omega)}$, $\|\mathbf{b}\|_{L^{\infty}(\Omega)},\|c\|_{L^{\infty}(\Omega)}$, and $\operatorname{diam}(\Omega)$.

\subsection{Reduction on refined elements}

As in Corollary 3.4 of Ref. 15, the assumptions (M1)-(M3), (R2)-(R3) and (S1) imply the existence of $C_{\text {red }} \geq 1$ and $0<q_{\text {red }}<1$ such that for all $\mathcal{T}_{\bullet} \in \mathbb{T}$ and all $\mathcal{T}_{0} \in \operatorname{refine}\left(\mathcal{T}_{\bullet}\right)$, it holds that

$$
\eta_{\circ}\left(\mathcal{T}_{\circ} \backslash \mathcal{T}_{\bullet}\right)^{2} \leq q_{\text {red }} \eta_{\bullet}\left(\mathcal{T}_{\bullet} \backslash \mathcal{T}_{\circ}\right)^{2}+C_{\text {red }}\left\|U_{\circ}-U_{\bullet}\right\|_{H^{1}(\Omega)}^{2} .
$$

The constants $C_{\text {red }}$ and $q_{\mathrm{red}}=q_{\mathrm{son}}{ }^{1 / d}$ depend only on (M1)-(M3), (R2)-(R3), (S1) as well as on $\|\mathbf{A}\|_{\mathbf{W}^{1, \infty}(\Omega)},\|\mathbf{b}\|_{\mathbf{L}^{\infty}(\Omega)},\|c\|_{L^{\infty}(\Omega)}$, and $\operatorname{diam}(\Omega)$. Note that $\left[\mathbf{A} \nabla U_{\bullet}\right.$. $\nu]=0$ on $\left(\partial T^{\prime} \backslash \partial T\right) \cap \Omega$ for all sons $T^{\prime} \varsubsetneqq T$ of an element $T \in \mathcal{T}_{\bullet}$, since $\left.U_{\bullet}\right|_{T} \in$ $H^{2}(T)$.

\subsection{Estimator reduction principle}

Choose sufficiently small $\delta>0$ such that $0<q_{\text {est }}:=(1+\delta)\left(1-\left(1-q_{\text {red }}\right) \theta\right)<1$ and define $C_{\text {est }}:=C_{\text {red }}+\left(1+\delta^{-1}\right) C_{\text {stab }}{ }^{2}$. With Secs. 4.44.5 elementary calculation 
shows that

$$
\eta_{\ell+1}^{2} \leq q_{\mathrm{est}} \eta_{\ell}^{2}+C_{\mathrm{est}}\left\|U_{\ell+1}-U_{\ell}\right\|_{H^{1}(\Omega)}^{2} \quad \text { for all } \ell \in \mathbb{N}_{0}
$$

see Lemma 4.7 of Ref. [14 Nestedness (S2) ensures that $\mathcal{X}_{\infty}:=\overline{\bigcup_{\ell \in \mathbb{N}_{0}} \mathcal{X}_{\ell}}$ is a closed subspace of $H_{0}^{1}(\Omega)$ and hence admits a unique Galerkin solution $U_{\infty} \in \mathcal{X}_{\infty}$. Note that $U_{\ell}$ is also a Galerkin approximation of $U_{\infty}$. Hence, the Céa lemma (2.7) with $u$ replaced by $U_{\infty}$ and a density argument prove $\left\|U_{\infty}-U_{\ell}\right\|_{H^{1}(\Omega)} \rightarrow 0$ as $\ell \rightarrow \infty$. Elementary calculus, estimator reduction (4.5), and Sec. 4.2 thus prove $\left\|u-U_{\ell}\right\|_{H^{1}(\Omega)} \lesssim \eta_{\ell} \rightarrow 0$ as $\ell \rightarrow \infty$. This proves $U_{\ell} \rightarrow U_{\infty}=u$; see Sec. 2 of Ref. 2 for the detailed argument.

\subsection{General quasi-orthogonality}

By use of reliability from Sec. 4.2 the plain convergence result from Sec. 4.6 and a perturbation argument (since the non-symmetric part of $\mathcal{L}$ is compact), it is shown in Proof of Theorem 8 of Ref. 21] that

$$
\begin{aligned}
& \sum_{k=\ell}^{N}\left(\left\|U_{k+1}-U_{k}\right\|_{H^{1}(\Omega)}^{2}-\varepsilon\left\|u-U_{k}\right\|_{H^{1}(\Omega)}^{2}\right) \\
& \quad \leq C(\varepsilon) \eta_{\ell}^{2} \text { for all } 0 \leq \ell \leq N \text { and all } \varepsilon>0 .
\end{aligned}
$$

The constant $C(\varepsilon)$ depends on $\varepsilon>0$, the operator $\mathcal{L}$, the sequence $\left(U_{\ell}\right)_{\ell \in \mathbb{N}_{0}}$, and $C_{\text {rel }}$. See also Proposition 6.1 of Ref. 14 for a short paraphrase of the proof.

Remark 4.1. If the bilinear form $\langle\cdot, \cdot\rangle_{\mathcal{L}}$ is symmetric, 44.6) follows from the Pythagoras theorem in the $\mathcal{L}$-induced energy norm $\|v\|_{\mathcal{L}}^{2}:=\langle v, v\rangle_{\mathcal{L}}$ and norm equivalence

$$
\begin{aligned}
\sum_{k=\ell}^{N}\left\|U_{k+1}-U_{k}\right\|_{H^{1}(\Omega)}^{2} \simeq \sum_{k=\ell}^{N}\left\|U_{k+1}-U_{k}\right\|_{\mathcal{L}}^{2} & =\left\|u-U_{\ell}\right\|_{\mathcal{L}}^{2}-\left\|u-U_{N}\right\|_{\mathcal{L}}^{2} \\
& \lesssim\left\|u-U_{\ell}\right\|_{H^{1}(\Omega)}^{2} .
\end{aligned}
$$

Together with reliability (2.14), this proves (4.6) even for $\varepsilon=0$, and $C(\varepsilon) \simeq C_{\text {rel }}^{2}$ is independent of the sequence $\left(U_{\ell}\right)_{\ell \in \mathbb{N}_{0}}$.

\subsection{Linear convergence with optimal rates}

The remaining claims (2.16)-2.17) follow from Theorem 4.1 of Ref. 14 which only relies on (R1), (2.3), (R4)-(R5), stability (Sec. 4.4), reduction (Sec. 4.5), quasiorthogonality (Sec. 4.7), discrete reliability (Sec. 4.1), and reliability (Sec. 4.2).

\section{Proof of Theorem 3.1}

\subsection{Admissibility and refine}

In this section, we show that, given a mesh $\widehat{\mathcal{T}}_{\bullet} \in \widehat{\mathbb{T}}$, iterative application of the refinement Algorithm 3.1 generates exactly the set of all admissible meshes $\widehat{\mathcal{T}}_{\circ}$ 
that are finer than $\widehat{\mathcal{T}}_{\bullet}$. In particular, this implies that $\widehat{\mathbb{T}}$ coincides with the set of all admissible hierarchical meshes that are finer than $\widehat{\mathcal{T}}_{0}$, which we have already mentioned in Sec. 3.5 We start with the following lemma.

Lemma 5.1. Let $\widehat{\mathcal{T}}_{\bullet}$ and $\widehat{\mathcal{T}}_{\circ}$ be hierarchical meshes such that $\widehat{\mathcal{T}}_{\circ}$ is finer than $\widehat{\mathcal{T}}_{\bullet}$, i.e. $\widehat{\Omega}_{\bullet}^{k} \subseteq \widehat{\Omega}_{\circ}^{k}$ for all $k \in \mathbb{N}_{0}$. Then, for all $\widehat{\beta}_{\circ} \in \widehat{\mathcal{H}}_{\circ}$ there exists $\widehat{\beta}_{\bullet} \in \widehat{\mathcal{H}}_{\bullet}$ with $\operatorname{supp}\left(\widehat{\beta}_{\circ}\right) \subseteq \operatorname{supp}\left(\widehat{\beta}_{\bullet}\right)$.

Proof. Clearly, we may assume $\widehat{\beta}_{\circ} \in \widehat{\mathcal{H}}_{\circ} \backslash \widehat{\mathcal{H}}_{\bullet}$. Let $k:=\operatorname{level}\left(\widehat{\beta}_{\circ}\right)$ and define $\widehat{\beta}^{k}:=$ $\widehat{\beta}_{\circ}$. Since $\widehat{\beta}^{k} \in \widehat{\mathcal{H}}_{\circ}$, 3.12 implies that $\operatorname{supp}\left(\widehat{\beta}^{k}\right) \backslash \widehat{\Omega}_{\circ}^{k+1} \neq \emptyset$ and $\operatorname{supp}\left(\widehat{\beta}^{k}\right) \subseteq \widehat{\Omega}_{\circ}^{k}$. Since $\widehat{\beta}^{k} \notin \widehat{\mathcal{H}}_{\bullet},(3.12)$ implies that $\operatorname{supp}\left(\widehat{\beta}^{k}\right) \backslash \widehat{\Omega}_{\bullet}^{k+1}=\emptyset$ or $\operatorname{supp}\left(\widehat{\beta}^{k}\right) \nsubseteq \widehat{\Omega}_{\bullet}^{k}$. However, $\widehat{\Omega}_{\bullet}^{k+1} \subseteq \widehat{\Omega}_{\circ}^{k+1}$ and $\operatorname{supp}\left(\widehat{\beta}^{k}\right) \backslash \widehat{\Omega}_{\circ}^{k+1} \neq \emptyset$ imply that $\operatorname{supp}\left(\widehat{\beta}^{k}\right) \backslash \widehat{\Omega}_{\bullet}^{k+1} \neq \emptyset$. Hence, we have $\operatorname{supp}\left(\widehat{\beta}^{k}\right) \nsubseteq \widehat{\Omega}_{\bullet}^{k}$, which especially implies $k>0$. This is equivalent to $\operatorname{supp}\left(\widehat{\beta}^{k}\right) \backslash \widehat{\Omega}_{\bullet}^{k} \neq \emptyset$. Clearly, there exists $\widehat{\beta}^{k-1} \in \mathcal{B}^{k-1}$ with $\operatorname{supp}\left(\widehat{\beta}^{k}\right) \subseteq \operatorname{supp}\left(\widehat{\beta}^{k-1}\right)$. If $\widehat{\beta}^{k-1} \in \widehat{\mathcal{H}}_{\bullet}$, we are done. Otherwise, (3.12) implies that $\operatorname{supp}\left(\widehat{\beta}^{k-1}\right) \backslash \widehat{\Omega}_{\bullet}^{k}=\emptyset$ or $\operatorname{supp}\left(\widehat{\beta}^{k-1}\right) \nsubseteq \widehat{\Omega}_{\bullet}^{k-1}$. Again, the first case is not possible because

$$
\operatorname{supp}\left(\widehat{\beta}^{k-1}\right) \backslash \widehat{\Omega}_{\bullet}^{k} \supseteq \operatorname{supp}\left(\widehat{\beta}^{k}\right) \backslash \widehat{\Omega}_{\bullet}^{k} \neq \emptyset .
$$

Hence, we have $\operatorname{supp}\left(\widehat{\beta}^{k-1}\right) \nsubseteq \widehat{\Omega}_{\bullet}^{k-1}$ which especially implies $k-1>0$. This is equivalent to $\operatorname{supp}\left(\widehat{\beta}^{k-1}\right) \backslash \widehat{\Omega}_{\bullet}^{k-1} \neq \emptyset$. Inductively, we obtain a sequence $\widehat{\beta}^{k}, \ldots, \widehat{\beta}^{K}$ with $\widehat{\beta}^{j} \in \mathcal{B}^{j}$ and $\operatorname{supp}\left(\widehat{\beta}^{K}\right) \supseteq \cdots \supseteq \operatorname{supp}\left(\widehat{\beta}^{k}\right)$, where $\widehat{\beta}^{K} \in \widehat{\mathcal{H}}$. for some $K \geq 0$.

Proposition 5.1. If $\widehat{\mathcal{T}}_{\bullet} \in \widehat{\mathbb{T}}$, then refine $\left(\widehat{\mathcal{T}}_{\bullet}\right)$ coincides with the set of all admissible hierarchical meshes $\widehat{\mathcal{T}}_{0} \in \widehat{\mathbb{T}}$ that are finer than $\widehat{\mathcal{T}}_{\bullet}$.

Proof. We prove the assertion in four steps.

Step 1: We show that $\widehat{\mathcal{T}}_{\circ}:=\operatorname{refine}\left(\widehat{\mathcal{T}}_{\bullet}, \widehat{\mathcal{M}}_{\bullet}\right) \in \widehat{\mathbb{T}}$ for any $\widehat{\mathcal{M}} \bullet \subseteq \widehat{\mathcal{T}}_{\bullet}$. Let $\widehat{T}, \widehat{T}^{\prime} \in \widehat{\mathcal{T}}_{\circ}$ with $\widehat{T}^{\prime} \in \mathcal{N}_{\circ}(\widehat{T})$, i.e. there exists $\widehat{\beta}_{\circ} \in \widehat{\mathcal{H}}_{\circ}$ with $\left|\widehat{T} \cap \operatorname{supp}\left(\widehat{\beta}_{\circ}\right)\right| \neq 0 \neq\left|\widehat{T}^{\prime} \cap \operatorname{supp}\left(\widehat{\beta}_{\circ}\right)\right|$; see (3.19). By Lemma 5.1, there exists some (not necessarily unique) $\widehat{\beta}_{\bullet} \in \widehat{\mathcal{H}}_{\bullet}$ with $\operatorname{supp}\left(\widehat{\beta}_{\circ}\right) \subseteq \operatorname{supp}\left(\widehat{\beta}_{\bullet}\right)$. We consider four different cases.

(i) Let $\widehat{T}, \widehat{T}^{\prime} \in \widehat{\mathcal{T}}_{\bullet}$. Then, $\left|\widehat{T} \cap \operatorname{supp}\left(\widehat{\beta}_{\bullet}\right)\right| \neq 0 \neq\left|\widehat{T}^{\prime} \cap \operatorname{supp}\left(\widehat{\beta}_{\bullet}\right)\right|$, i.e. $\widehat{T}^{\prime} \in \mathcal{N}_{\bullet}(\widehat{T})$ and hence $\left|\operatorname{level}(\widehat{T})-\operatorname{level}\left(\widehat{T}^{\prime}\right)\right| \leq 1$ by $\widehat{\mathcal{T}}_{\bullet} \in \widehat{\mathbb{T}}$.

(ii) Let $\widehat{T}, \widehat{T}^{\prime} \in \widehat{\mathcal{T}}_{\circ} \backslash \widehat{\mathcal{T}}_{\bullet}$. Let $\widehat{T}_{\bullet}, \widehat{T}_{\bullet}^{\prime} \in \widehat{\mathcal{T}}_{\bullet}$ with $\widehat{T} \varsubsetneqq \widehat{T}_{\bullet}, \widehat{T}^{\prime} \varsubsetneqq \widehat{T}_{\bullet}^{\prime}$. Then, it holds that $\operatorname{level}(\widehat{T})=\operatorname{level}\left(\widehat{T}_{\bullet}\right)+1, \operatorname{level}\left(\widehat{T}^{\prime}\right)=\operatorname{level}\left(\widehat{T}_{\bullet}^{\prime}\right)+1$ as well as $\left|\widehat{T}_{\bullet} \cap \operatorname{supp}\left(\widehat{\beta}_{\bullet}\right)\right| \neq$ $0 \neq\left|\widehat{T}_{\bullet}^{\prime} \cap \operatorname{supp}\left(\widehat{\beta}_{\bullet}\right)\right|$. By definition, it follows that $\widehat{T}_{\bullet}^{\prime} \in \mathcal{N}_{\bullet}\left(\widehat{T}_{\bullet}\right)$ and hence $\left|\operatorname{level}(\widehat{T})-\operatorname{level}\left(\widehat{T}^{\prime}\right)\right|=\left|\operatorname{level}\left(\widehat{T}_{\bullet}\right)-\operatorname{level}\left(\widehat{T}_{\bullet}^{\prime}\right)\right| \leq 1$ by $\widehat{\mathcal{T}}_{\bullet} \in \widehat{\mathbb{T}}$.

(iii) Let $\widehat{T} \in \widehat{\mathcal{T}}_{\bullet} \backslash \widehat{\mathcal{T}}_{\bullet}, \widehat{T}^{\prime} \in \widehat{\mathcal{T}}_{\bullet}$. Let $\widehat{T}_{\bullet} \in \widehat{\mathcal{T}}_{\bullet}$ with $\widehat{T} \varsubsetneqq \widehat{T}_{\bullet}$. Then, $\left|\widehat{T}_{\bullet} \cap \operatorname{supp}\left(\widehat{\beta}_{\bullet}\right)\right| \neq$ $0 \neq\left|\widehat{T}^{\prime} \cap \operatorname{supp}\left(\widehat{\beta}_{\bullet}\right)\right|$, and $\left|\operatorname{level}\left(\widehat{T}_{\bullet}\right)-\operatorname{level}\left(\widehat{T}^{\prime}\right)\right| \leq 1$ by $\widehat{\mathcal{T}}_{\bullet} \in \widehat{\mathbb{T}}$. We argue by contradiction and assume $\left|\operatorname{level}(\widehat{T})-\operatorname{level}\left(\widehat{T}^{\prime}\right)\right|>1$. Together with level $\left(\widehat{T}_{\bullet}\right)+$ 
$1=\operatorname{level}(\widehat{T})$, this yields $\operatorname{level}\left(\widehat{T}_{\bullet}\right)-1=\operatorname{level}\left(\widehat{T}^{\prime}\right)$. Hence, $\widehat{T}^{\prime} \in \mathcal{N}_{\bullet}^{\text {bad }}\left(\widehat{T}_{\bullet}\right)$ with $\widehat{T}_{\bullet} \in \widehat{\mathcal{M}}_{\bullet}^{(\text {end })}$. By Algorithm 3.1(i), we get $\widehat{T}^{\prime} \in \widehat{\mathcal{M}}_{\bullet}^{\text {(end) }}$. This contradicts $\widehat{T}^{\prime} \in \widehat{\mathcal{T}}_{\bullet}$ and hence proves $\left|\operatorname{level}(\widehat{T})-\operatorname{level}\left(\widehat{T}^{\prime}\right)\right| \leq 1$.

(iv) Let $\widehat{T} \in \widehat{\mathcal{T}}_{\bullet}, \widehat{T}^{\prime} \in \widehat{\mathcal{T}}_{\circ} \backslash \widehat{\mathcal{T}}_{\bullet}$. Since $\widehat{T}^{\prime} \in \mathcal{N}_{\bullet}(\widehat{T})$ is equivalent to $\widehat{T} \in \mathcal{N}_{\bullet}\left(\widehat{T}^{\prime}\right)$, we argue as in (iii) to conclude $\left|\operatorname{level}(\widehat{T})-\operatorname{level}\left(\widehat{T}^{\prime}\right)\right| \leq 1$.

Step 2: It is clear that an arbitrary $\widehat{\mathcal{T}}_{\circ} \in \operatorname{refine}\left(\widehat{\mathcal{T}}_{\bullet}\right)$ is finer than $\widehat{\mathcal{T}}_{\bullet}$. By induction, Step 1 proves the inclusion refine $\left(\widehat{\mathcal{T}}_{\bullet}\right) \subseteq \widehat{\mathbb{T}}$.

Step 3: To prove the converse inclusion, let $\widehat{\mathcal{T}}_{\circ} \in \widehat{\mathbb{T}}$ be an admissible mesh that is finer than $\widehat{\mathcal{T}}_{\bullet}$. Moreover, let $\widehat{T} \in \widehat{\mathcal{T}}_{\bullet} \backslash \widehat{\mathcal{T}}_{\circ}$. We show that $\widehat{\mathcal{T}}_{\circ}$ is also finer than $\widehat{\mathcal{T}}_{\star}:=\operatorname{refine}\left(\widehat{\mathcal{T}}_{\bullet},\{\widehat{T}\}\right)$. We argue by contradiction and suppose that $\widehat{\mathcal{T}}_{\circ}$ is not finer than $\widehat{\mathcal{T}}_{\star}$. Since ref ine bisects each element of $\widehat{\mathcal{T}}_{\bullet}$ at most once, there exists a refined element $\widehat{T}^{(0)} \in \widehat{\mathcal{T}}_{\bullet} \backslash \widehat{\mathcal{T}}_{\star}$ which is also in $\widehat{\mathcal{T}}_{\circ}$, i.e. $\widehat{T}^{(0)} \in\left(\widehat{\mathcal{T}}_{\bullet} \backslash \widehat{\mathcal{T}}_{\star}\right) \cap \widehat{\mathcal{T}}_{\circ}$. In particular, $\widehat{T}^{(0)} \neq \widehat{T} \in \widehat{\mathcal{T}}_{\bullet} \backslash \widehat{\mathcal{T}}_{\circ}$. Thus, Algorithm 3.1 shows that $\widehat{T}^{(0)} \in \mathcal{N}_{\bullet}^{\text {bad }}\left(\widehat{T}^{(1)}\right)$ for some $\widehat{T}^{(1)} \in \widehat{\mathcal{T}}_{\bullet} \backslash \widehat{\mathcal{T}}_{\star}$. If $\widehat{T}^{(1)} \in \widehat{\mathcal{T}}_{\circ}$ and hence $\widehat{T}^{(1)} \in\left(\widehat{\mathcal{T}}_{\bullet} \backslash \widehat{\mathcal{T}}_{\star}\right) \cap \widehat{\mathcal{T}}_{\mathrm{o}}$, we have again $\widehat{T}^{(1)} \neq \widehat{T}$ as well as $\widehat{T}^{(1)} \in \mathcal{N}_{\bullet}^{\text {bad }}\left(\widehat{T}^{(2)}\right)$ for some $\widehat{T}^{(2)} \in \widehat{\mathcal{T}}_{\bullet} \backslash \widehat{\mathcal{T}}_{\star}$. Inductively, we see the existence of $\widehat{T}^{(J-1)} \in\left(\widehat{\mathcal{T}}_{\bullet} \backslash \widehat{\mathcal{T}}_{\star}\right) \cap \widehat{\mathcal{T}}_{\circ}$ such that $\widehat{T}^{(J-1)} \in \mathcal{N}_{\bullet}^{\operatorname{bad}}\left(\widehat{T}^{(J)}\right)$ for some $\widehat{T}^{(J)} \in \widehat{\mathcal{T}}_{\bullet} \backslash \widehat{\mathcal{T}}_{\star}$ with $\widehat{T}^{(J)} \notin \widehat{\mathcal{T}}_{\circ}$. In particular, this implies the existence of $\widehat{T}_{\circ}^{(J)} \in \widehat{\mathcal{T}}_{\circ}$ with $\widehat{T}_{\circ}^{(J)} \varsubsetneqq \widehat{T}^{(J)}$.

By definition of $\mathcal{N}_{\bullet}^{\text {bad }}(\cdot)$, we have $\widehat{T}^{(J)}, \widehat{T}^{(J-1)} \subseteq \operatorname{supp}(\widehat{\beta})$ for some $\widehat{\beta} \in \widehat{\mathcal{H}}_{\bullet}$ as well as level $\left(\widehat{T}^{(J-1)}\right)=\operatorname{level}\left(\widehat{T}^{(J)}\right)-1$. Hence, (3.15) and $\widehat{\mathcal{T}}_{\bullet} \in \widehat{\mathbb{T}}$ show $k:=$ $\operatorname{level}(\widehat{\beta})=\operatorname{level}\left(\widehat{T}^{(J-1)}\right)$. Since $\widehat{T}^{(J-1)} \in \widehat{\mathcal{T}}_{\circ}$, (3.10) implies $\widehat{T}^{(J-1)} \not \widehat{\Omega}_{\circ}^{k+1}$ and hence $\operatorname{supp}(\widehat{\beta}) \nsubseteq \widehat{\Omega}_{\circ}^{k+1}$. Moreover, (3.12) shows $\operatorname{supp}(\widehat{\beta}) \subseteq \widehat{\Omega}_{\bullet}^{k} \subseteq \widehat{\Omega}_{\circ}^{k}$. Using (3.12) again, we see $\widehat{\beta} \in \widehat{\mathcal{H}}_{\circ}$. Together with $\widehat{T}_{\circ}^{(J)}, \widehat{T}^{(J-1)} \subseteq \operatorname{supp}(\widehat{\beta})$ and $\operatorname{level}\left(\widehat{T}_{\circ}^{(J)}\right) \geq$ $\operatorname{level}\left(\widehat{T}^{(J)}\right)+1=\operatorname{level}\left(\widehat{T}^{(J-1)}\right)+2$, this contradicts admissibility of $\widehat{\mathcal{T}}_{\circ} \in \widehat{\mathbb{T}}$, and concludes the proof.

Step 4: Let again $\widehat{\mathcal{T}}_{\circ} \in \widehat{\mathbb{T}}$ be an arbitrary admissible mesh that is finer than $\widehat{\mathcal{T}}_{\bullet}$. Step 3 together with Step 2 shows that we can iteratively refine $\widehat{\mathcal{T}}_{\bullet}$ and obtain a sequence $\widehat{\mathcal{T}}_{(0)}, \ldots, \widehat{\mathcal{T}}_{(J)}$ with $\widehat{\mathcal{T}}_{\bullet}=\widehat{\mathcal{T}}_{(0)}, \widehat{\mathcal{T}}_{(j+1)}=\operatorname{refine}\left(\widehat{\mathcal{T}}_{(j)},\left\{\widehat{T}_{(j)}\right\}\right)$ with some $\widehat{T}_{(j)} \in \widehat{\mathcal{T}}_{(j)} \backslash \widehat{\mathcal{T}}_{(j+1)}$ for $j=1, \ldots, J-1$ and $\widehat{\mathcal{T}}_{(J)}=\widehat{\mathcal{T}}_{\circ}$. By definition, this proves $\widehat{\mathcal{T}}_{\circ} \in \operatorname{refine}\left(\widehat{\mathcal{T}}_{\bullet}\right)$.

\subsection{Verification of (M1)-(M4)}

The mesh properties (M1)-(M3) essentially follow from admissibility in the sense of Sec. 3.4 in combination with the following lemma.

Lemma 5.2. Let $\widehat{\mathcal{T}}_{\bullet}$ be an arbitrary hierarchical mesh in the parameter domain. Then,

$$
\Pi_{\bullet}(\widehat{T}) \subseteq \mathcal{N}_{\bullet}(\widehat{T}) \quad \text { for all } \widehat{T} \in \widehat{\mathcal{T}}_{\bullet}
$$


Proof. Let $\widehat{T}^{\prime} \in \Pi_{\bullet}(\widehat{T})$, i.e. $\widehat{T}^{\prime} \in \widehat{\mathcal{T}}$ • with $\widehat{T} \cap \widehat{T}^{\prime} \neq \emptyset$. We abbreviate $k:=\operatorname{level}(\widehat{T})$. Since all knot multiplicities are smaller than $p+1$, there exists $\widehat{\beta}^{k} \in \widehat{\mathcal{B}}^{k}$ such that $\left|\widehat{T} \cap \operatorname{supp}\left(\widehat{\beta}^{k}\right)\right| \neq 0 \neq\left|\widehat{T}^{\prime} \cap \operatorname{supp}\left(\widehat{\beta}^{k}\right)\right|$. If $\widehat{\beta}^{k} \in \widehat{\mathcal{H}}_{\bullet}$, then $\widehat{T}^{\prime} \in \widehat{\mathcal{N}}_{\bullet}(\widehat{T})$. If $\widehat{\beta}^{k} \notin \widehat{\mathcal{H}} \cdot$, the characterization (3.12) shows that $\operatorname{supp}\left(\widehat{\beta}^{k}\right) \not \subseteq \widehat{\Omega}_{\bullet}^{k}$ or $\operatorname{supp}\left(\widehat{\beta}^{k}\right) \subseteq \widehat{\Omega}_{\bullet}^{k+1}$. By choice of $k$, it holds that $\widehat{T} \subseteq \operatorname{supp}\left(\widehat{\beta}^{k}\right)$. In view of (3.10), $\widehat{T} \in \widehat{\mathcal{T}}_{\bullet}$ implies $\widehat{T} \not \widehat{\Omega}_{\bullet}^{k+1}$. Hence, $\operatorname{supp}\left(\widehat{\beta}^{k}\right) \not \nsubseteq \widehat{\Omega}_{\bullet}^{k}$ and, in particular, $k>0$. Next, there exists $\widehat{\beta}^{k-1} \in \widehat{\mathcal{B}}^{k-1}$ such that $\operatorname{supp}\left(\widehat{\beta}^{k}\right) \subseteq \operatorname{supp}\left(\widehat{\beta}^{k-1}\right)$. If $\widehat{\beta}^{k-1} \in \widehat{\mathcal{H}}_{\bullet}$, then $\widehat{T}^{\prime} \in \widehat{\mathcal{N}}_{\bullet}(\widehat{T})$. If $\widehat{\beta}^{k-1} \notin \widehat{\mathcal{H}}_{\bullet}$, there holds again either $\operatorname{supp}\left(\widehat{\beta}^{k-1}\right) \nsubseteq \widehat{\Omega}_{\bullet}^{k-1}$ or $\operatorname{supp}\left(\widehat{\beta}^{k-1}\right) \subseteq \widehat{\Omega}_{\bullet}^{k}$. Due to $\operatorname{supp}\left(\widehat{\beta}^{k}\right) \nsubseteq \widehat{\Omega}_{\bullet}^{k}$, the second case is not possible. Hence, $\operatorname{supp}\left(\widehat{\beta}^{k-1}\right) \not \subset \widehat{\Omega}_{\bullet}^{k-1}$ and, in particular, $k-1>0$. We proceed in the same way to get a sequence $\widehat{\beta}^{k}, \ldots, \widehat{\beta}^{K}$ with $\widehat{\beta}^{j} \in \widehat{\mathcal{B}}^{j}$ and $\operatorname{supp}\left(\widehat{\beta}^{K}\right) \supseteq \cdots \supseteq \operatorname{supp}\left(\widehat{\beta}^{k}\right)$, where $\widehat{\beta}^{K} \in \widehat{\mathcal{H}}$. for some $K \geq 0$.

We define the patches $\pi_{\bullet}(\cdot)$ and $\Pi_{\bullet}(\cdot)$ in the parameter domain analogously to the patches in the physical domain, see Sec. 2.1.

With Lemma 5.2, one can easily verify that $\mathbb{T}$ satisfies (M1)-(M3): let $\mathcal{T}_{\bullet} \in \mathbb{T}$. We start with (M1). Let $T \in \mathcal{T}_{\bullet}$ and $T^{\prime} \in \Pi_{\bullet}(T)$. Lemma 5.2 and admissibility show for the corresponding elements $\widehat{T}, \widehat{T}^{\prime}$ in the parameter domain that $\mid \operatorname{level}(\widehat{T})-$ level $\left(\widehat{T}^{\prime}\right) \mid \leq 1$, wherefore $|\widehat{T}| \simeq\left|\widehat{T}^{\prime}\right|$. Regularity (3.28) of the transformation $\gamma$ finally yields $|T| \simeq\left|T^{\prime}\right|$. The constant $C_{\text {shape }}$ depends only on $d, C_{\gamma}$ and $\mathcal{T}_{0}$.

To prove (M2), let $T \in \mathcal{T}_{\bullet}$ and $T^{\prime} \in \Pi_{\bullet}(T)$. As before, we have $\mid$ level $(\widehat{T})-$ $\operatorname{level}\left(\widehat{T}^{\prime}\right) \mid \leq 1$ for the corresponding elements in the parameter domain. With this, one easily sees that $\# \Pi$ • $(T) \leq C_{\text {patch }}$ with a constant $C_{\text {patch }}>0$ that depends only on the dimension $d$.

Regularity (3.28) of $\gamma$ shows that it is sufficient to prove (M3) for hyperrectangles $\widehat{T}$ in the parameter domain. There, the trace inequality (M3) is wellknown; see, e.g. Satz 3.4.5 of Ref. 20. The constant $C_{\text {trace }}$ depends only on $d, C_{\gamma}$ and $\mathcal{T}_{0}$.

Finally, (M4) in the parameter domain follows immediately from the Poincaré inequality. By regularity (3.28) of $\gamma$, this property transfers to the physical domain. The constant $C_{\text {dual }}$ depends only on $d, C_{\gamma}$ and $\mathcal{T}_{0}$.

\subsection{Verification of (R1)-(R3)}

Let $\mathcal{T}_{\bullet} \in \mathbb{T}, \mathcal{T}_{\circ} \in \operatorname{refine}\left(\mathcal{T}_{\bullet}\right)$, and $T \in \mathcal{T}_{\bullet}$. (R1) is trivially satisfied with $C_{\text {son }}=2^{d}$, since each refined element is split into exactly $2^{d}$ elements. Moreover, the union of sons property (R2) holds by definition.

To see the reduction property (R3), let $T^{\prime} \in \mathcal{T}_{\circ}$ with $T^{\prime} \varsubsetneqq T$. Since each refined element is split it into $2^{d}$ elements, we have for the corresponding elements in the parameter domain $\left|\widehat{T}^{\prime}\right| \leq 2^{-d}|\widehat{T}|$. Next, we prove $\left|T^{\prime}\right| \leq q_{\text {son }}|T|$ with a constant $0<q_{\text {son }}<1$ which depends only on $d$ and $C_{\gamma}$. Indeed, we even prove for arbitrary measurable sets $\widehat{S}^{\prime} \subseteq \widehat{S} \subseteq \overline{\widehat{\Omega}}$ and $S:=\gamma(\widehat{S}), S^{\prime}:=\gamma\left(\widehat{S}^{\prime}\right)$ that $0<\left|\widehat{S}^{\prime}\right| \leq 2^{-d}|\widehat{S}|$ implies $\left|S^{\prime}\right| \leq q_{\text {son }}|S|$. To see this, we argue by contradiction and assume that 
there are two sequences of such sets $\left(\widehat{S}_{n}\right)_{n \in \mathbb{N}}$ and $\left(\widehat{S}_{n}^{\prime}\right)_{n \in \mathbb{N}}$ with $\left|S_{n}^{\prime}\right| /\left|S_{n}\right| \rightarrow 1$. This implies $\left|S_{n} \backslash S_{n}^{\prime}\right| /\left|S_{n}\right| \rightarrow 0$ and yields the contradiction

$$
1-2^{-d} \leq \frac{\left|\widehat{S}_{n} \backslash \widehat{S}_{n}^{\prime}\right|}{\left|\widehat{S}_{n}\right|} \simeq \frac{\int_{\widehat{S}_{n} \backslash \widehat{S}_{n}^{\prime}}|\operatorname{det} D \gamma(t)| d t}{\int_{\widehat{S}_{n}}|\operatorname{det} D \gamma(t)| d t}=\frac{\left|S_{n} \backslash S_{n}^{\prime}\right|}{\left|S_{n}\right|} \stackrel{n \rightarrow \infty}{\longrightarrow} 0 .
$$

\subsection{Verification of (R4)}

The proof of the closure estimate (R4) goes back to the seminal works. $\underline{843}$ Our analysis builds on Sec. 3 of Ref. 13 which proves (R4) for the refinement strategy of Ref. 11] see also Remark 3.2. The following auxiliary result states that refine $(\cdot, \cdot)$ is equivalent to iterative refinement of one single element. For a mesh in the parameter domain $\widehat{\mathcal{T}}_{\bullet} \in \widehat{\mathbb{T}}$ and an arbitrary set $\widehat{\mathcal{M}}_{\bullet}$, we define refine $\left(\widehat{\mathcal{T}}_{\bullet}, \widehat{\mathcal{M}}_{\bullet}\right):=$ refine $\left(\widehat{\mathcal{T}}_{\bullet}, \widehat{\mathcal{M}}_{\bullet} \cap \widehat{\mathcal{T}}_{\bullet}\right)$ and note that refine $\left(\widehat{\mathcal{T}}_{\bullet}, \emptyset\right)=\widehat{\mathcal{T}}_{\bullet}$.

Lemma 5.3. Let $\widehat{\mathcal{T}}_{\bullet} \in \widehat{\mathbb{T}}$ and $\widehat{\mathcal{M}}_{\bullet}=\left\{\widehat{T}_{1}, \ldots, \widehat{T}_{n}\right\} \subseteq \widehat{\mathcal{T}}_{\bullet}$. Then, it holds that

$$
\operatorname{refine}\left(\widehat{\mathcal{T}}_{\bullet}, \widehat{\mathcal{M}}_{\bullet}\right)=\operatorname{refine}\left(\operatorname{refine}\left(\ldots \operatorname{refine}\left(\widehat{\mathcal{T}}_{\bullet},\left\{\widehat{T}_{1}\right\}\right) \ldots,\left\{\widehat{T}_{n-1}\right\}\right),\left\{\widehat{T}_{n}\right\}\right)
$$

Proof. We only show that $\operatorname{refine}\left(\widehat{\mathcal{T}}_{\bullet}, \widehat{\mathcal{M}}_{\bullet}\right)=\operatorname{refine}\left(\operatorname{refine}\left(\widehat{\mathcal{T}}_{\bullet},\left\{\widehat{T}_{1}\right\}\right), \widehat{\mathcal{M}}_{\bullet} \backslash\right.$ $\left\{\widehat{T}_{1}\right\}$ ), and then (5.2) follows by induction. We define:

$$
\begin{aligned}
& \widehat{\mathcal{T}}_{(1)}:=\operatorname{refine}\left(\widehat{\mathcal{T}}_{\bullet},\left\{\widehat{T}_{1}\right\}\right), \quad \widehat{\mathcal{T}}_{(2)}:=\operatorname{refine}\left(\widehat{\mathcal{T}}_{(1)}, \widehat{\mathcal{M}}_{\bullet} \backslash\left\{\widehat{T}_{1}\right\}\right), \\
& \widehat{\mathcal{M}}_{(0)}:=\widehat{\mathcal{M}}_{(0)}^{(0)}:=\left\{\widehat{T}_{1}\right\}, \quad \widehat{\mathcal{M}}_{(1)}:=\widehat{\mathcal{M}}_{(1)}^{(0)}:=\widetilde{\mathcal{M}}_{(1)}:=\widetilde{\mathcal{M}}_{(1)}^{(0)}:=\widehat{\mathcal{M}}_{\bullet} \backslash\left\{\widehat{T}_{1}\right\} .
\end{aligned}
$$

For $i \in \mathbb{N}_{0}$, we introduce the following notation which is conform with that of Algorithm 3.1:

$$
\begin{aligned}
& \widehat{\mathcal{M}}_{(0)}^{(i+1)}:=\widehat{\mathcal{M}}_{(0)}^{(i)} \cup \underset{\widehat{T} \in \widehat{\mathcal{M}}_{(0)}^{(i)}}{\bigcup} \mathcal{N}_{\bullet}^{\mathrm{bad}}(\widehat{T}), \quad \widehat{\mathcal{M}}_{(1)}^{(i+1)}:=\widehat{\mathcal{M}}_{(1)}^{(i)} \cup \underset{T_{\in} \widehat{\mathcal{M}}_{(1)}^{(i)}}{\bigcup} \mathcal{N}_{(1)}^{\text {bad }}(\widehat{T}), \\
& \widetilde{\mathcal{M}}_{(1)}^{(i+1)}:=\widetilde{\mathcal{M}}_{(1)}^{(i)} \cup \underset{\widehat{T} \in \widetilde{\mathcal{M}}_{(1)}^{(i)}}{\bigcup_{\bullet}^{\text {bad }}(\widehat{T}) .}
\end{aligned}
$$

Finally, we set

$$
\widehat{\mathcal{M}}_{(0)}^{(\text {end })}:=\bigcup_{i \in \mathbb{N}_{0}} \widehat{\mathcal{M}}_{(0)}^{(i)}, \quad \widehat{\mathcal{M}}_{(1)}^{(\mathrm{end})}:=\bigcup_{i \in \mathbb{N}_{0}} \widehat{\mathcal{M}}_{(1)}^{(i)}, \quad \widetilde{\mathcal{M}}_{(1)}^{(\text {end })}:=\bigcup_{i \in \mathbb{N}_{0}} \widetilde{\mathcal{M}}_{(1)}^{(i)} .
$$

With these notations, we have:

$$
\begin{aligned}
\widehat{\mathcal{T}}_{\bullet} \backslash \widehat{\mathcal{T}}_{(1)}= & \widehat{\mathcal{M}}_{(0)}^{\text {(end) }}, \quad \widehat{\mathcal{T}}_{(1)} \backslash \widehat{\mathcal{T}}_{(2)}=\widehat{\mathcal{M}}_{(1)}^{(\text {end })} \\
& \widehat{\mathcal{T}}_{\bullet} \backslash \operatorname{refine}\left(\widehat{\mathcal{T}}_{\bullet}, \widehat{\mathcal{M}}_{\bullet}\right)=\widehat{\mathcal{M}}_{(0)}^{(\text {end })} \cup \widetilde{\mathcal{M}}_{(1)}^{(\text {end })} .
\end{aligned}
$$

To conclude the proof, we will prove that $\widehat{\mathcal{M}}_{(0)}^{(\text {end })} \cup \widehat{\mathcal{M}}_{(1)}^{\text {(end) }}=\widehat{\mathcal{M}}_{(0)}^{(\text {end })} \cup \widetilde{\mathcal{M}}_{(1)}^{(\text {end })}$. To this end, we split the proof into three steps.

Step 1: We first prove $\widehat{\mathcal{M}}_{(1)}^{(\text {end })} \subseteq \widehat{\mathcal{T}}_{\bullet}$ by induction. Clearly, we have $\widehat{\mathcal{M}}_{(1)}^{(0)} \subseteq \widehat{\mathcal{T}}_{\bullet}$. Now, let $i \in \mathbb{N}_{0}$ and suppose $\widehat{\mathcal{M}}_{(1)}^{(i)} \subseteq \widehat{\mathcal{T}}_{\bullet}$. To see $\widehat{\mathcal{M}}_{(1)}^{(i+1)} \subseteq \widehat{\mathcal{T}}_{\bullet}$, we argue by contradiction 
and assume that there exists $\widehat{T} \in \widehat{\mathcal{M}}_{(1)}^{(i)}$ and $\widehat{T}^{\prime} \in \mathcal{N}_{(1)}^{\text {bad }}(\widehat{T}) \backslash \widehat{\mathcal{T}}_{\bullet}$. By Lemma 5.1, the unique father element $\widehat{T}_{\bullet}^{\prime} \in \widehat{\mathcal{T}}_{\bullet}$ with $\widehat{T}^{\prime} \varsubsetneqq \widehat{T}_{\bullet}^{\prime}$ satisfies $\widehat{T}_{\bullet}^{\prime} \in \mathcal{N}_{\bullet}(\widehat{T})$. Therefore, admissibility of $\widehat{\mathcal{T}}_{\bullet}$ proves $\left|\operatorname{level}(\widehat{T})-\operatorname{level}\left(\widehat{T}_{\bullet}^{\prime}\right)\right| \leq 1$, which contradicts

$$
\operatorname{level}\left(\widehat{T}_{\bullet}^{\prime}\right)=\operatorname{level}\left(\widehat{T}^{\prime}\right)-1=\operatorname{level}(\widehat{T})-2 .
$$

Step 2: Let $\widehat{T} \in \widehat{\mathcal{M}}_{(1)}^{(\text {end })}$. In this step, we will prove that

$$
\widehat{\mathcal{M}}_{0}^{(\text {end })} \cup \mathcal{N}_{(1)}^{\text {bad }}(\widehat{T})=\widehat{\mathcal{M}}_{(0)}^{(\text {end })} \cup \mathcal{N}_{\bullet}^{\text {bad }}(\widehat{T}) .
$$

By Step 1, we have $\widehat{T} \in \widehat{\mathcal{T}}_{\bullet}$. Lemma 5.1 proves $\mathcal{N}_{(1)}^{\text {bad }}(\widehat{T}) \cap \widehat{\mathcal{T}}_{\bullet} \subseteq \mathcal{N}_{\bullet}^{\text {bad }}(\widehat{T})$. Using Step 1 again, we see $\mathcal{N}_{(1)}^{\text {bad }}(\widehat{T}) \subseteq \widehat{\mathcal{M}}_{(1)}^{(\text {end })} \subseteq \widehat{\mathcal{T}}_{\bullet}$ and conclude " $\subseteq$ " in (5.3). To see

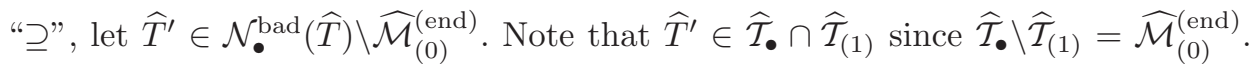
There exists $\widehat{\beta} \in \widehat{\mathcal{H}}_{\bullet}$ with $\widehat{T}, \widehat{T}^{\prime} \subseteq \operatorname{supp}(\widehat{\beta})$. By admissibility of $\widehat{\mathcal{T}}_{\bullet} \in \widehat{\mathbb{T}}$, $\operatorname{level}\left(\widehat{T}^{\prime}\right)=$ $\operatorname{level}(\widehat{T})-1$, and 3.15), we see that level $(\widehat{\beta})=\operatorname{level}\left(\widehat{T}^{\prime}\right)=: k^{\prime}$. Hence, 3.12 yields that $\operatorname{supp}(\widehat{\beta}) \subseteq \widehat{\Omega}_{\bullet}^{k^{\prime}}$ as well as $\operatorname{supp}(\widehat{\beta}) \nsubseteq \widehat{\Omega}_{\bullet}^{k^{\prime}+1}$. The definition of $k^{\prime}$ and $(3.10)$ show that $\widehat{T}^{\prime} \nsubseteq \widehat{\Omega}_{(1)}^{k^{\prime}+1}$. We conclude $\operatorname{supp}(\widehat{\beta}) \subseteq \widehat{\Omega}_{\bullet}^{k^{\prime}} \subseteq \widehat{\Omega}_{(1)}^{k^{\prime}}$ and $\operatorname{supp}(\widehat{\beta}) \nsubseteq \widehat{\Omega}_{(1)}^{k^{\prime}+1}$, since $\widehat{\mathcal{T}}_{(1)} \ni \widehat{T}^{\prime} \subseteq \operatorname{supp}(\widehat{\beta})$. Therefore, (3.12) shows $\widehat{\beta} \in \widehat{\mathcal{H}}_{(1)}$. Altogether, we have $\widehat{T}^{\prime} \in \mathcal{N}_{(1)}^{\operatorname{bad}}(\widehat{T})$.

Step 3: Finally, we prove $\widehat{\mathcal{M}}_{(0)}^{(\text {end })} \cup \widehat{\mathcal{M}}_{(1)}^{(i)}=\widehat{\mathcal{M}}_{(0)}^{(\text {end })} \cup \widetilde{\mathcal{M}}_{(1)}^{(i)}$ by induction on $i \in \mathbb{N}_{0}$. In particular, this will imply $\widehat{\mathcal{M}}_{(0)}^{(\text {end })} \cup \widehat{\mathcal{M}}_{(1)}^{(\text {end })}=\widehat{\mathcal{M}}_{(0)}^{(\text {end })} \cup \widetilde{\mathcal{M}}_{(1)}^{(\text {end })}$. For $i=0$, the claim follows from $\widehat{\mathcal{M}}_{(1)}^{(0)}=\widetilde{\mathcal{M}}_{(1)}^{(0)}$. By Step 2, the induction step works as follows:

$$
\begin{aligned}
\widehat{\mathcal{M}}_{(0)}^{(\text {end })} \cup \widehat{\mathcal{M}}_{(1)}^{(i+1)} & =\widehat{\mathcal{M}}_{(0)}^{(\text {end })} \cup \widehat{\mathcal{M}}_{(1)}^{(i)} \cup \bigcup_{\widehat{T} \in \widehat{\mathcal{M}}_{(1)}^{(i)}} \mathcal{N}_{(1)}^{\text {bad }}(\widehat{T}) \\
& \stackrel{5.3}{=} \widehat{\mathcal{M}}_{(0)}^{(\text {end })} \cup \widehat{\mathcal{M}}_{(1)}^{(i)} \cup \bigcup_{\widehat{T} \in \widehat{\mathcal{M}}_{(0)}^{\text {end })} \cup \widehat{\mathcal{M}}_{(1)}^{(i)}} \mathcal{N}_{\bullet}^{\text {bad }}(\widehat{T}) \\
& =\widehat{\mathcal{M}}_{(0)}^{(\text {end })} \cup \widetilde{\mathcal{M}}_{(1)}^{(i)} \cup \bigcup_{\widehat{T} \in \widehat{\mathcal{M}}_{(0)}^{\text {end })} \cup \widetilde{\mathcal{M}}_{(1)}^{(i)}} \mathcal{N}_{\bullet}^{\text {bad }}(\widehat{T}) \\
& =\widehat{\mathcal{M}}_{(0)}^{(\text {end })} \cup \widetilde{\mathcal{M}}_{(1)}^{(i+1)} .
\end{aligned}
$$

This concludes the proof.

Let $\widehat{\mathcal{T}}_{\bullet} \in \widehat{\mathbb{T}}$. For $\widehat{T}, \widehat{T}^{\prime} \in \widehat{\mathcal{T}}_{\bullet}$, let $\operatorname{dist}\left(\widehat{T}, \widehat{T}^{\prime}\right)$ be the Euclidean distance of their midpoints in the parameter domain. Let $\widehat{T} \in \widehat{\mathcal{T}}_{\bullet}$ and $\widehat{T}^{\prime} \in \mathcal{N}_{\bullet}(\widehat{T})$. Hence, there is $\widehat{\beta} \in \widehat{\mathcal{H}}_{\bullet}$ such that $\widehat{T}, \widehat{T}^{\prime} \subseteq \operatorname{supp}(\beta)$. In particular, it holds that $\operatorname{dist}\left(\widehat{T}, \widehat{T}^{\prime}\right) \leq \operatorname{diam}(\operatorname{supp}(\widehat{\beta}))$. By admissibility of $\widehat{\mathcal{T}}_{\bullet}$ and (3.15), we see $|\operatorname{level}(\widehat{\beta})-\operatorname{level}(\widehat{T})| \leq 1$. This proves

$$
\operatorname{dist}\left(\widehat{T}, \widehat{T}^{\prime}\right) \leq C_{\operatorname{diam}} 2^{-\operatorname{level}(\widehat{T})},
$$


where $C_{\text {diam }}>0$ depends only on $d, \widehat{\mathcal{T}}_{0}$ and $\left(p_{1}, \ldots, p_{d}\right)$. With this observation, we can prove the following lemma. The proof follows the lines of Lemma 11 of Ref. 13], but is also included here for completeness.

Lemma 5.4. Let $\widehat{\mathcal{T}}_{\bullet} \in \widehat{\mathbb{T}}$ and $\widehat{T}^{\prime} \in \widehat{\mathcal{T}}_{\bullet}$. With $\widehat{\mathcal{T}}_{\circ}=\operatorname{refine}\left(\widehat{\mathcal{T}}_{\bullet},\left\{\widehat{T}^{\prime}\right\}\right)$, it holds that

$$
\operatorname{dist}\left(\widehat{T}, \widehat{T}^{\prime}\right) \leq 2^{-\operatorname{level}(\widehat{T})} C_{\text {dist }} \quad \text { for all } \widehat{T} \in \widehat{\mathcal{T}}_{\circ} \backslash \widehat{\mathcal{T}}_{\bullet},
$$

where $C_{\text {dist }}>0$ depends only on $d, \widehat{\mathcal{T}}_{0}$ and $\left(p_{1}, \ldots, p_{d}\right)$.

Proof. $\widehat{T} \in \widehat{\mathcal{T}}_{\circ} \backslash \widehat{\mathcal{T}}_{\bullet}$ implies the existence of a sequence $\widehat{T}^{\prime}=\widehat{T}_{J}, \widehat{T}_{J-1}, \ldots, \widehat{T}_{0}$ such that $\widehat{T}_{j-1} \in \mathcal{N}_{\bullet}^{\operatorname{bad}}\left(\widehat{T}_{j}\right)$ and $\widehat{T}$ is a child of $\widehat{T}_{0}$, i.e. $\widehat{T} \varsubsetneqq \widehat{T}_{0}$ and level $(\widehat{T})=\operatorname{level}\left(\widehat{T}_{0}\right)+1$. Since level $\left(\widehat{T}_{j-1}\right)=\operatorname{level}\left(\widehat{T}_{j}\right)-1$, it follows

$$
\operatorname{level}\left(\widehat{T}_{j}\right)=\operatorname{level}\left(\widehat{T}_{0}\right)+j .
$$

The triangle inequality proves

$$
\operatorname{dist}\left(\widehat{T}, \widehat{T}^{\prime}\right) \leq \operatorname{dist}\left(\widehat{T}, \widehat{T}_{0}\right)+\operatorname{dist}\left(\widehat{T}_{0}, \widehat{T}^{\prime}\right) \leq \operatorname{dist}\left(\widehat{T}, \widehat{T}_{0}\right)+\sum_{j=1}^{J} \operatorname{dist}\left(\widehat{T}_{j}, \widehat{T}_{j-1}\right) .
$$

Further, there exists a constant $C>0$ which depends only on $\widehat{\mathcal{T}}_{0}$ and $d$, such that

$$
\operatorname{dist}\left(\widehat{T}, \widehat{T}_{0}\right) \leq C 2^{-\operatorname{level}(\widehat{T})} \text {. }
$$

With (5.4) and (5.6), we see

$$
\begin{aligned}
\sum_{j=1}^{J} \operatorname{dist}\left(\widehat{T}_{j}, \widehat{T}_{j-1}\right) \stackrel{(5.4)}{\leq} C_{\operatorname{diam}} \sum_{j=1}^{J} 2^{-\operatorname{level}\left(\widehat{T}_{j}\right)} \stackrel{\sqrt[5.6)]{=}}{=} C_{\operatorname{diam}} \sum_{j=1}^{J} 2^{-\operatorname{level}\left(\widehat{T}_{0}\right)-j} \\
\leq C_{\operatorname{diam}} 2^{-\operatorname{level}(\widehat{T})-1}
\end{aligned}
$$

which concludes the proof.

Finally, let $\widehat{\mathcal{T}}_{\bullet} \in \widehat{\mathbb{T}}$ and $\widehat{T} \in \widehat{\mathcal{T}}_{\bullet}$. We abbreviate $\widehat{\mathcal{T}}_{\circ}=\operatorname{refine}\left(\widehat{\mathcal{T}}_{\bullet},\{\widehat{T}\}\right)$. Then, there holds

$$
\operatorname{level}\left(\widehat{T}^{\prime}\right) \leq \operatorname{level}(\widehat{T})+1 \quad \text { for all refined elements } \widehat{T}^{\prime} \in \widehat{\mathcal{T}}_{\circ} \backslash \widehat{\mathcal{T}}_{\bullet}
$$

To see this, note that all elements $\widehat{T}^{\prime \prime} \in \widehat{\mathcal{T}}_{\bullet} \backslash \widehat{\mathcal{T}}_{\circ}$ which are refined, satisfy $\widehat{T}^{\prime \prime}=\widehat{T}$ or $\operatorname{level}\left(\widehat{T}^{\prime \prime}\right) \leq \operatorname{level}(\widehat{T})-1$. Therefore, their children satisfy level $\left(\widehat{T}^{\prime}\right) \leq \operatorname{level}(\widehat{T})+1$. With this last observation, we can argue as in the proof of Theorem 12 of Ref. 13 to show the closure estimate (R4). The constant $C_{\text {clos }}>0$ depends only on $d, \widehat{\mathcal{T}_{0}}$, and $\left(p_{1}, \ldots, p_{d}\right)$.

\subsection{Verification of (R5)}

We prove (R5) in the parameter domain $\widehat{\Omega}$. Let $\widehat{\mathcal{T}}_{\bullet}, \widehat{\mathcal{T}}_{\star} \in \widehat{\mathbb{T}}$ be two admissible hierarchical meshes. We define the overlay

$$
\widehat{\mathcal{T}}_{\circ}:=\left\{\widehat{T}_{\bullet} \in \widehat{\mathcal{T}}_{\bullet}: \exists \widehat{T}_{\star} \in \widehat{\mathcal{T}}_{\star}, \widehat{T}_{\bullet} \subseteq \widehat{T}_{\star}\right\} \cup\left\{\widehat{T}_{\star} \in \widehat{\mathcal{T}}_{\star}: \exists \widehat{T}_{\bullet} \in \widehat{\mathcal{T}}_{\bullet}, \widehat{T}_{\star} \subseteq \widehat{T}_{\bullet}\right\}
$$


Note that $\widehat{\mathcal{T}}_{\circ}$ is a hierarchical mesh with hierarchical domains $\widehat{\Omega}_{\circ}^{k}=\widehat{\Omega}_{\bullet}^{k} \cup \widehat{\Omega}_{\star}^{k}$ for $k \in \mathbb{N}_{0}$. In particular, $\widehat{\mathcal{T}}_{\circ}$ is finer than $\widehat{\mathcal{T}}_{\bullet}$ and $\widehat{\mathcal{T}}_{\star}$. Moreover, the overlay estimate easily follows from the definition of $\widehat{\mathcal{T}}_{\circ}$. It remains to prove that $\widehat{\mathcal{T}}_{\circ}$ is admissible. To see this, let $\widehat{T}, \widehat{T}^{\prime} \in \widehat{\mathcal{T}}_{\circ}$ with $\widehat{T}^{\prime} \in \mathcal{N}_{\circ}(\widehat{T})$, i.e. there exists $\widehat{\beta}_{\circ} \in \widehat{\mathcal{H}}_{\circ}$ such that $\mid \widehat{T} \cap$ $\operatorname{supp}\left(\widehat{\beta}_{\circ}\right)|\neq 0 \neq| \widehat{T}^{\prime} \cap \operatorname{supp}\left(\widehat{\beta}_{\circ}\right) \mid$. Without loss of generality, we suppose level $(\widehat{T}) \geq$ level $\left(\widehat{T}^{\prime}\right)$ and $\widehat{T} \in \widehat{\mathcal{T}}_{\bullet}$. If $\widehat{T}^{\prime} \in \widehat{\mathcal{T}}_{\bullet}$, Lemma 5.1 shows $\widehat{T}^{\prime} \in \mathcal{N}_{\bullet}(\widehat{T})$, and admissibility of $\widehat{\mathcal{T}}_{\text {• implies }}\left|\operatorname{level}(\widehat{T})-\operatorname{level}\left(\widehat{T}^{\prime}\right)\right| \leq 1$. Now, let $\widehat{T}^{\prime} \in \widehat{\mathcal{T}}_{\star}$. By definition of the overlay, there exists $\widehat{T}_{\bullet}^{\prime} \in \widehat{\mathcal{T}}_{\bullet}$ with $\widehat{T}^{\prime} \subseteq \widehat{T}_{\bullet}^{\prime}$ and $\operatorname{level}\left(\widehat{T}_{\bullet}^{\prime}\right) \leq \operatorname{level}\left(\widehat{T}^{\prime}\right)$. Further, Lemma 5.1 provides some (not necessarily unique) $\widehat{\beta}_{\bullet} \in \widehat{\mathcal{H}}_{\bullet} \operatorname{such}$ that $\operatorname{supp}\left(\widehat{\beta}_{\circ}\right) \subseteq \operatorname{supp}\left(\widehat{\beta}_{\bullet}\right)$. Hence, $\left|\widehat{T} \cap \operatorname{supp}\left(\widehat{\beta}_{\bullet}\right)\right| \neq 0 \neq\left|\widehat{T}_{\bullet}^{\prime} \cap \operatorname{supp}\left(\widehat{\beta}_{\bullet}\right)\right|$, i.e. $\widehat{T}_{\bullet}^{\prime} \in \mathcal{N}_{\bullet}(\widehat{T})$. Since $\widehat{\mathcal{T}}_{\bullet} \in \widehat{\mathbb{T}}$, it follows that $\left|\operatorname{level}(\widehat{T})-\operatorname{level}\left(\widehat{T}_{\bullet}^{\prime}\right)\right| \leq 1$. Altogether, we see that

$$
\begin{aligned}
\left|\operatorname{level}(\widehat{T})-\operatorname{level}\left(\widehat{T}^{\prime}\right)\right|=\operatorname{level}(\widehat{T})-\operatorname{level}\left(\widehat{T}^{\prime}\right) & \leq \operatorname{level}(\widehat{T})-\operatorname{level}\left(\widehat{T}_{\bullet}^{\prime}\right) \\
& \leq\left|\operatorname{level}(\widehat{T})-\operatorname{level}\left(\widehat{T}_{\bullet}^{\prime}\right)\right| \leq 1 .
\end{aligned}
$$

This concludes the proof of (R5).

\subsection{Verification of (S1)}

Let $T \in \mathcal{T}_{\bullet} \in \mathbb{T}$. Let $V_{\bullet} \in \mathcal{X}_{\bullet}$. Define $\widehat{V}_{\bullet}:=V_{\bullet} \circ \gamma \in \widehat{\mathcal{X}}_{\bullet} \subseteq \widehat{\mathcal{Y}}_{\bullet}$ and $\widehat{T}:=\gamma^{-1}(T) \in \widehat{\mathcal{T}}_{\bullet}$. Regularity (3.28) of $\gamma$ proves for $i \in\{0,1,2\}$ :

$$
\left\|V_{\bullet}\right\|_{H^{i}(T)} \simeq\left\|\widehat{V}_{\bullet}\right\|_{H^{i}(\widehat{T})},
$$

where the hidden constants depend only on $d$ and $C_{\gamma}$. Since $\widehat{V}_{\bullet}$ is a $\widehat{\mathcal{T}}_{\bullet}$-piecewise tensor polynomial, there holds for $i, j \in\{0,1,2\}$ with $j \leq i$ that

$$
|\widehat{T}|^{(i-j) / d}\left\|\widehat{V}_{\bullet}\right\|_{H^{i}(\widehat{T})} \lesssim\left\|\widehat{V}_{\bullet}\right\|_{H^{j}(\widehat{T})},
$$

where the hidden constant depends only on $d, \widehat{\mathcal{T}}_{0}$, and $\left(p_{1}, \ldots, p_{d}\right)$. Together, ([5.9)(5.10) conclude the proof of (S1), where $C_{\text {inv }}$ depends only on $d, C_{\gamma}, \widehat{\mathcal{T}}_{0}$, and $\left(p_{1}, \ldots, p_{d}\right)$.

\subsection{Verification of (S2)}

In (3.21), we already saw that $\mathcal{T}_{\circ} \in \operatorname{refine}\left(\mathcal{T}_{\bullet}\right)$ with $\mathcal{T}_{\bullet} \in \mathbb{T}$ implies nestedness of the corresponding ansatz spaces $\mathcal{X}_{\bullet} \subseteq \mathcal{X}_{\circ}$.

\subsection{Verification of (S3)}

We show the assertion in the parameter domain. For arbitrary but fixed $k_{\text {proj }} \in \mathbb{N}_{0}$ (which will be fixed later in Sec. 5.10 to be $k_{\text {proj }}:=2(p+1)$ ), we set $k_{\text {loc }}:=$ $k_{\text {proj }}+2(p+1)$. Let $\widehat{\mathcal{T}}_{\bullet} \in \widehat{\mathbb{T}}, \widehat{\mathcal{T}}_{\circ} \in \operatorname{refine}\left(\widehat{\mathcal{T}}_{\bullet}\right)$, and $\widehat{V}_{\circ} \in \widehat{\mathcal{X}}_{\circ}$. We define the patch functions $\pi_{\bullet}$ and $\Pi_{\bullet}$ in the parameter domain analogously to the patch functions in the physical domain, see Sec. 2.1 Let $\widehat{T} \in \widehat{\mathcal{T}}_{\bullet} \backslash \Pi_{\bullet}^{\text {loc }}\left(\widehat{\mathcal{T}}_{\bullet} \backslash \widehat{\mathcal{T}}_{\circ}\right)$, where $\Pi_{\bullet}^{\text {loc }}:=\Pi_{\bullet}^{k_{\text {loc }}}$. 
First, we show that

$$
\Pi_{\bullet}^{\mathrm{loc}}(\widehat{T}) \subseteq \widehat{\mathcal{T}}_{\bullet} \cap \widehat{\mathcal{T}}_{\circ}
$$

To this end, we argue by contradiction and assume that there exists $\widehat{T}^{\prime} \in \Pi_{\bullet}^{\text {loc }}(\widehat{T})$ with $\widehat{T}^{\prime} \notin \widehat{\mathcal{T}}_{\bullet} \cap \widehat{\mathcal{T}}_{\circ}$. This is equivalent to $\widehat{T} \in \Pi_{\bullet}^{\mathrm{loc}}\left(\widehat{T}^{\prime}\right)$ and $\widehat{T}^{\prime} \in \widehat{\mathcal{T}}_{\bullet} \backslash \widehat{\mathcal{T}}_{\mathrm{o}}$. This implies $\widehat{T} \in \Pi_{\bullet}^{\text {loc }}\left(\widehat{\mathcal{T}}_{\bullet} \backslash \widehat{\mathcal{T}}_{\circ}\right)$, contradicts $\widehat{T} \in \widehat{\mathcal{T}}_{\bullet} \backslash \Pi_{\bullet}^{\text {loc }}\left(\widehat{\mathcal{T}}_{\bullet} \backslash \widehat{\mathcal{T}}_{\circ}\right)$, and hence proves (5.11). Again, we abbreviate $\pi_{\bullet}^{\text {proj }}:=\pi_{\bullet}^{k_{\text {proj }}}$. According to Corollary 3.1 it holds that

$$
\begin{aligned}
\left\{\left.\widehat{V}_{\bullet}\right|_{\pi_{\bullet}^{\text {proj }}(\widehat{T})}: \widehat{V}_{\bullet}\right. & \left.\in \widehat{\mathcal{X}}_{\bullet}\right\}=\operatorname{span}\left\{\left.\widehat{\beta}\right|_{\pi_{\bullet}^{\text {proj }}(T)}: \widehat{\beta} \in \widehat{\mathcal{H}}_{\bullet}\right. \\
\left.\wedge \widehat{\beta}\right|_{\partial \widehat{\Omega}} & \left.=0 \wedge\left|\operatorname{supp}(\widehat{\beta}) \cap \pi_{\bullet}^{\operatorname{proj}}(\widehat{T})\right|>0\right\},
\end{aligned}
$$

as well as

$$
\begin{aligned}
\left\{\left.\widehat{V}_{\circ}\right|_{\pi_{\bullet}^{\mathrm{proj}}(T)}: \widehat{V}_{\circ}\right. & \left.\in \widehat{\mathcal{X}}_{\circ}\right\}=\operatorname{span}\left\{\left.\widehat{\beta}\right|_{\pi_{\bullet}^{\mathrm{proj}}(T)}: \widehat{\beta} \in \widehat{\mathcal{H}}_{\circ}\right. \\
\left.\wedge \widehat{\beta}\right|_{\partial \widehat{\Omega}} & \left.=0 \wedge\left|\operatorname{supp}(\widehat{\beta}) \cap \pi_{\bullet}^{\operatorname{proj}}(\widehat{T})\right|>0\right\} .
\end{aligned}
$$

We will prove

$$
\left\{\widehat{\beta} \in \widehat{\mathcal{H}}_{\bullet}:\left|\operatorname{supp}(\widehat{\beta}) \cap \pi_{\bullet}^{\operatorname{proj}}(\widehat{T})\right|>0\right\}=\left\{\widehat{\beta} \in \widehat{\mathcal{H}}_{\circ}:\left|\operatorname{supp}(\widehat{\beta}) \cap \pi_{\bullet}^{\operatorname{proj}}(\widehat{T})\right|>0\right\},
$$

which will conclude (S3). First let $\widehat{\beta}$ be an element of the left set. By Remark 3.1 this implies $\operatorname{supp}(\widehat{\beta}) \subseteq \pi_{\bullet}^{\text {loc }}(\widehat{T})$. Together with (5.11), we see $\operatorname{supp}(\widehat{\beta}) \subseteq \bigcup\left(\widehat{\mathcal{T}}_{\bullet} \cap \widehat{\mathcal{T}}_{0}\right)$. This proves that no element within $\operatorname{supp}(\widehat{\beta})$ is changed during refinement, i.e. $\widehat{\Omega}_{\bullet}^{k} \cap$ $\operatorname{supp}(\widehat{\beta})=\widehat{\Omega}_{\circ}^{k} \cap \operatorname{supp}(\widehat{\beta})$ for all $k \in \mathbb{N}_{0}$. Thus, (3.12) proves $\widehat{\beta} \in \widehat{\mathcal{H}}_{\circ}$. The proof works the same if we start with some $\widehat{\beta}$ in the right set. This proves (5.12) and therefore (S3).

\subsection{Truncated hierarchical B-splines}

We will define some Scott-Zhang-type operator $J_{\bullet}$ similarly as in Ref. 41 with the help of so-called truncated hierarchical B-splines (THB-splines) introduced in Ref. 26. In this section, we recall their definition and list some basic properties. For a more detailed presentation, we refer to, e.g. Refs. 26 and 41

Let $\widehat{\mathcal{T}} \bullet$ be an arbitrary hierarchical mesh in the parameter domain. For $k \in \mathbb{N}_{0}$, we define the truncation trunc ${ }^{k+1}: \widehat{\mathcal{Y}}^{k} \rightarrow \widehat{\mathcal{Y}}^{k+1}$ as follows:

$$
\operatorname{trunc}_{\bullet}^{k+1}\left(\widehat{V}^{k}\right):=\sum_{\substack{\widehat{\beta} \in \widehat{\mathcal{B}}^{k+1} \\ \operatorname{supp}(\widehat{\beta}) \mathbb{Z}^{k+1}}} c_{\widehat{\beta}} \widehat{\beta} \text { for all } \widehat{V}^{k}=\sum_{\widehat{\beta} \in \widehat{\mathcal{B}}^{k+1}} c_{\widehat{\beta}} \widehat{\beta} \in \widehat{\mathcal{Y}}^{k} \subset \widehat{\mathcal{Y}}^{k+1}
$$

i.e. truncation is defined via the (unique) basis representation of $\widehat{V}^{k} \in \widehat{\mathcal{Y}}^{k}$ with respect $\widehat{\mathcal{B}}^{k+1}$. Recall that $M_{\bullet} \in \mathbb{N}$ is the minimal integer such that $\widehat{\Omega}_{\bullet}^{M \bullet}=\emptyset$. For all $\widehat{\beta} \in \widehat{\mathcal{H}}_{\bullet}$, the corresponding truncated hierarchical B-spline reads

$$
\text { Trunc } \bullet(\widehat{\beta}):=\operatorname{trunc}_{\bullet}^{M \bullet-1}\left(\operatorname{trunc}_{\bullet}^{M \bullet-2}\left(\ldots\left(\operatorname{trunc} \bullet_{\bullet}^{\operatorname{level}(\widehat{\beta})+1}(\widehat{\beta})\right) \ldots\right)\right) \text {. }
$$


As the set $\widehat{\mathcal{H}}_{\bullet}$, the set of THB-splines $\left\{\operatorname{Trunc}_{\bullet}(\widehat{\beta}): \widehat{\beta} \in \widehat{\mathcal{H}}_{\bullet}\right\}$ forms a basis of the space of hierarchical splines $\widehat{\mathcal{Y}}_{\bullet}$. In Sec. 3.1, we mentioned that each basis function in $\widehat{\mathcal{B}}^{k}$ is the linear combination of basis functions $\widehat{\mathcal{B}}^{k+1}$, where the corresponding coefficients are non-negative; see (3.5). For $\widehat{\beta} \in \widehat{\mathcal{H}}_{\bullet}$, this proves

$$
0 \leq \operatorname{Trunc}_{\bullet}(\widehat{\beta}) \leq \widehat{\beta} \leq 1,
$$

and in particular $\operatorname{supp}\left(\operatorname{Trunc}_{\bullet}(\widehat{\beta})\right) \subseteq \operatorname{supp}(\widehat{\beta})$. With this and the fact that the THB-splines are a basis of $\widehat{\mathcal{Y}}_{\bullet}$, Corollary 3.1 proves

$$
\widehat{\mathcal{X}}_{\bullet}=\operatorname{span}\left\{\operatorname{Trunc}_{\bullet}(\widehat{\beta}):\left.\widehat{\beta} \in \widehat{\mathcal{H}}_{\bullet} \wedge \widehat{\beta}\right|_{\partial \widehat{\Omega}}=0\right\},
$$

where the set on the right-hand side is even a basis of $\widehat{\mathcal{X}}_{\bullet}$. The following proposition shows that for an admissible mesh $\widehat{\mathcal{T}}_{\bullet} \in \widehat{\mathcal{T}}_{\bullet}$, the full truncation Trunc reduces to

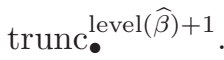

Proposition 5.2. Let $\widehat{\mathcal{T}}_{\bullet} \in \widehat{\mathbb{T}}$ and $\widehat{\beta} \in \widehat{\mathcal{H}}_{\bullet}$. Then, it holds that

$$
\operatorname{Trunc}_{\bullet}(\widehat{\beta})=\operatorname{trunc}_{\bullet}^{\operatorname{level}(\widehat{\beta})+1}(\widehat{\beta}) \text {. }
$$

Proof. We prove the proposition in two steps.

Step 1: Let $k^{\prime}<k^{\prime \prime} \in \mathbb{N}_{0}$ and $\widehat{\beta}^{\prime} \in \widehat{\mathcal{B}}^{k^{\prime}}$ with representation $\widehat{\beta}^{\prime}=\sum_{\widehat{\beta}^{\prime \prime} \in \widehat{\mathcal{B}}^{k^{\prime \prime}}} c_{\widehat{\beta^{\prime \prime}}} \widehat{\beta}^{\prime \prime}$. Let $\widehat{\beta}^{\prime \prime} \in \widehat{\mathcal{B}}^{k^{\prime \prime}}$ such that $c_{\widehat{\beta}^{\prime \prime}} \neq 0$. Then, local linear independence (with the open set $\left.O=(0,1)^{d} \backslash \operatorname{supp}\left(\widehat{\beta}^{\prime}\right)\right)$ of $\widehat{\mathcal{B}}^{k^{\prime \prime}}$ implies $\operatorname{supp}\left(\widehat{\beta}^{\prime \prime}\right) \subseteq \operatorname{supp}\left(\widehat{\beta}^{\prime}\right)$.

Step 2: We prove (5.17). We abbreviate $k=\operatorname{level}(\widehat{\beta})$. Let $\widehat{\beta}=\sum_{\widehat{\beta}^{\prime} \in \widehat{\mathcal{B}}^{k+1}} c_{\widehat{\beta}^{\prime}} \widehat{\beta}^{\prime}$. Let $\widehat{\beta}^{\prime} \in \widehat{\mathcal{B}}^{k+1}$ with $\operatorname{supp}\left(\widehat{\beta}^{\prime}\right) \nsubseteq \widehat{\Omega}_{\bullet}^{k+1}$ and $c_{\widehat{\beta}^{\prime}} \neq 0$. By Step 1 , this proves $\operatorname{supp}\left(\widehat{\beta}^{\prime}\right) \subseteq$ $\operatorname{supp}(\widehat{\beta})$. For $k^{\prime \prime}>k+1$, we consider the representation

$$
\operatorname{trunc} c_{\bullet}^{k^{\prime \prime}}\left(\widehat{\beta}^{\prime}\right)=\sum_{\substack{\widehat{\beta}^{\prime \prime} \in \widehat{\mathcal{B}}^{k^{\prime \prime}} \\ \operatorname{supp}\left(\widehat{\beta}^{\prime \prime}\right) \notin \notin \widehat{\Omega}_{\bullet}^{k^{\prime \prime}}}} c_{\widehat{\beta}^{\prime \prime}} \widehat{\beta}^{\prime \prime}, \quad \text { where } \quad \widehat{\beta}^{\prime}=\sum_{\widehat{\beta}^{\prime \prime} \in \widehat{\mathcal{B}}^{\prime \prime}} c_{\widehat{\beta^{\prime \prime}}}, \widehat{\beta}^{\prime \prime} .
$$

If $\widehat{\beta}^{\prime \prime} \in \widehat{\mathcal{B}}^{k^{\prime \prime}}$ with $\operatorname{supp}\left(\widehat{\beta}^{\prime \prime}\right) \subseteq \widehat{\Omega}_{\bullet}^{k^{\prime \prime}}$, let $\widehat{T}^{\prime \prime} \in \widehat{\mathcal{T}}^{k^{\prime \prime}}$ with $\widehat{T}^{\prime \prime} \subseteq \operatorname{supp}\left(\widehat{\beta}^{\prime \prime}\right)$. (3.10) shows the existence of an element $\widehat{T} \in \widehat{\mathcal{T}}$ • with $\operatorname{level}(\widehat{T}) \geq k^{\prime \prime}$ such that $\widehat{T} \subseteq$ $\widehat{T}^{\prime \prime}$. To see $c_{\widehat{\beta}^{\prime \prime}}=0$, we argue by contradiction and assume $c_{\widehat{\beta}^{\prime \prime}} \neq 0$. By Step 1 , this implies $\widehat{T} \subseteq \operatorname{supp}\left(\widehat{\beta}^{\prime \prime}\right) \subseteq \operatorname{supp}\left(\widehat{\beta}^{\prime}\right) \subseteq \operatorname{supp}(\widehat{\beta})$. Due to (3.15), this contradicts admissibility of $\widehat{\mathcal{T}}_{\bullet}$. This proves $c_{\widehat{\beta}^{\prime \prime}}=0$. Overall, we conclude trunc $k_{\bullet}^{\prime \prime}\left(\widehat{\beta}^{\prime}\right)=\widehat{\beta}^{\prime}$, and thus trunc$c_{\bullet}^{k^{\prime \prime}}\left(\operatorname{trunc}_{\bullet}^{k+1}(\widehat{\beta})\right)=\operatorname{trunc}_{\bullet}^{k+1}(\widehat{\beta})$ as well as (5.17).

Remark 5.1. Actually, the proposed refinement strategy of Algorithm 3.1 was designed for hierarchical B-splines; see also Proposition 3.2. However, 5.15) implies that Proposition 3.2 holds accordingly for truncated hierarchical B-splines. Moreover, if one applies the refinement strategy of Algorithm 3.1, (5.17) shows that the computation of the truncated hierarchical B-splines greatly simplifies. 


\subsection{Verification of (S4)-(S6)}

Given $\mathcal{T}_{\bullet} \in \mathbb{T}$, we are finally able to introduce a suitable Scott-Zhang-type operator $J_{\bullet}: H_{0}^{1}(\Omega) \rightarrow \mathcal{X}_{\bullet}$ which satisfies (S4)-(S6). To this end, it is sufficient to construct a corresponding operator $\widehat{J}_{\bullet}: H_{0}^{1}(\widehat{\Omega}) \rightarrow \widehat{\mathcal{X}}_{\bullet}$ in the parameter space, and to define

$$
J_{\bullet} v:=\left(\widehat{J}_{\bullet}(v \circ \gamma)\right) \circ \gamma^{-1} \text { for all } v \in H_{0}^{1}(\Omega) .
$$

By regularity (3.28) of $\gamma$, the properties (S4)-(S6) immediately transfer from the parameter domain $\widehat{\Omega}$ to the physical domain $\Omega$. Recall that $\widehat{\mathcal{B}}^{k} \cap \widehat{\mathcal{B}}^{k^{\prime}}=\emptyset$ for $k \neq k^{\prime}$. For $k \in \mathbb{N}_{0}$ and $\widehat{\beta} \in \widehat{\mathcal{B}}^{k}$, let $\widehat{T}_{\widehat{\beta}} \in \widehat{\mathcal{T}}^{k}$ be an arbitrary but fixed element with $\widehat{T}_{\widehat{\beta}} \subseteq \operatorname{supp}(\widehat{\beta})$. If $\widehat{\beta} \in \widehat{\mathcal{H}}_{\bullet}$, we additionally require ${ }^{\dagger} \widehat{T}_{\widehat{\beta}} \in \widehat{\mathcal{T}}_{\bullet}$, which is possible due to (3.15). By local linear independence and continuity of $\widehat{\mathcal{B}}^{k}$ (see Sec. 3.1), also the restricted basis functions $\left\{\left.\widehat{\beta}\right|_{\widehat{T}_{\widehat{\beta}}}:\left.\widehat{\beta} \in \widehat{\mathcal{B}}^{k} \wedge \widehat{\beta}\right|_{\widehat{T}_{\widehat{\beta}}} \neq 0\right\}$ are linearly independent. Hence, the Riesz theorem guarantees the existence and uniqueness of some $\widehat{\beta}^{*} \in$ $\left\{\left.\widehat{V}^{k}\right|_{\widehat{T}_{\widehat{\beta}}}: \widehat{V}^{k} \in \widehat{\mathcal{Y}}^{k}\right\}$ such that

$$
\int_{\widehat{T}_{\widehat{\beta}}} \widehat{\beta}^{*} \widehat{\beta}^{\prime} d t=\delta_{\widehat{\beta}, \widehat{\beta}^{\prime}} \quad \text { for all } \widehat{\beta}^{\prime} \in \widehat{\mathcal{B}}^{k}
$$

These dual basis functions $\widehat{\beta}^{*}$ satisfy the following scaling property.

Lemma 5.5. There exists $C_{\mathrm{dual}}>0$ such that for all $k \in \mathbb{N}_{0}$ and all $\widehat{\beta} \in \widehat{\mathcal{B}}^{k}$, it holds that

$$
\left\|\widehat{\beta}^{*}\right\|_{L^{\infty}\left(\widehat{T}_{\widehat{\beta}}\right)} \leq C_{\text {dual }}\left|\widehat{T}_{\widehat{\beta}}\right|^{-1}
$$

The constant $C_{\mathrm{dual}}$ depends only on $d, \widehat{\mathcal{T}}_{0}$ and $\left(p_{1}, \ldots, p_{d}\right)$.

Proof. Recall that $\widehat{T}_{\widehat{\beta}}$ is a rectangle of the form $\widehat{T}_{\ell_{1}, \ldots, \ell_{d}}^{k}=\left[t_{1, \ell_{1}}^{k}, t_{1, \ell_{1}+1}^{k}\right] \times \cdots \times$ $\left[t_{d, \ell_{d}}^{k}, t_{d, \ell_{d}+1}^{k}\right]$. We abbreviate $C:=\left|\widehat{T}_{\widehat{\beta}}\right|^{1 / d},\left(a_{1}, \ldots, a_{d}\right):=\left(t_{1, \ell_{1}}^{k}, \ldots, t_{d, \ell_{d}}^{k}\right)$ and define the normalized element $\widetilde{T}_{\widehat{\beta}}:=\left(\widehat{T}_{\widehat{\beta}}-\left(a_{1}, \ldots, a_{d}\right)\right) / C$ and the corresponding affine transformation $\Phi: \widetilde{T}_{\widehat{\beta}} \rightarrow \widehat{T}_{\widehat{\beta}}$. We apply the transformation formula to see $\int_{\widehat{T}_{\widehat{\beta}}} \widehat{\beta}^{*} \widehat{\beta}^{\prime} d t=C^{d} \int_{\widetilde{T}_{\widehat{\beta}}}\left(\widehat{\beta}^{*} \circ \Phi\right)(\widehat{\beta} \circ \Phi) d t$. Therefore, the Riesz theorem implies that $\widehat{\beta}^{*}=\left(\widetilde{\beta}^{*} \circ \Phi^{-1}\right) / C^{d}$, where $\widetilde{\beta}^{*}$ is the unique element in $\operatorname{span}\left(\widetilde{\mathcal{B}}^{k}\right)$ with $\widetilde{\mathcal{B}}^{k}:=\left\{\widehat{\beta}^{\prime} \circ \Phi: \widehat{\beta}^{\prime} \in \widehat{\mathcal{B}}^{k}\right\} \backslash\{0\}$ such that

$$
\int_{\widetilde{T}_{\widehat{\beta}}} \widetilde{\beta}^{*} \widetilde{\beta}^{\prime} d t=\delta_{\widetilde{\beta}, \widetilde{\beta^{\prime}}} \quad \text { for all } \widetilde{\beta}^{\prime} \in \widetilde{\mathcal{B}}^{k} .
$$

\footnotetext{
†Therefore, the elements $\widehat{T}_{\widehat{\beta}}$ depend additionally on the considered mesh $\widehat{\mathcal{T}}_{\bullet}$.
} 
By (3.3), each $\widetilde{\beta}^{\prime} \in \widetilde{\mathcal{B}}^{k}$ is of the form

$$
\widetilde{\beta}^{\prime}\left(\widetilde{s}_{1}, \ldots, \widetilde{s}_{d}\right)=\prod_{i=1}^{d} B\left(s_{i} \mid t_{i, j_{i}}^{k}, \ldots, t_{i, j_{i}+p_{i}+1}^{k}\right)
$$

with $\left(s_{1}, \ldots, s_{d}\right)=\left(\widetilde{s}_{1}, \ldots, \widetilde{s}_{d}\right) C+\left(a_{1}, \ldots, a_{d}\right)$.

We only have to consider $\widetilde{\beta}^{\prime}$ that are supported on $\widetilde{T}_{\widehat{\beta}}$. As the support of any $B\left(\cdot \mid t_{i, j_{i}}^{k}, \ldots, t_{i, j_{i}+p_{i}+1}^{k}\right)$ is just $\left[t_{i, j_{i}}^{k}, t_{i, j_{i}+p_{i}+1}^{k}\right]$, it is sufficient to consider $j_{i}=\ell_{i}-p_{i}, \ldots, \ell_{i}$. By the definition of B-splines, one immediately sees that an affine transformation in the parameter domain can just be passed to the knots, i.e.

$$
B\left(s_{i} \mid t_{i, j_{i}}^{k}, \ldots, t_{i, j_{i}+p_{i}+1}^{k}\right)=B\left(\widetilde{s}_{i} \mid\left(t_{i, j_{i}}^{k}-a_{1}\right) / C, \ldots,\left(t_{i, j_{i}+p_{i}+1}^{k}-a_{d}\right) / C\right) .
$$

Altogether, we see that $\widetilde{\beta}^{*}$ depends only on the knots

$$
\left(\frac{t_{i, j_{i}}^{k}-a_{1}}{C}, \ldots, \frac{t_{i, j_{i}+p_{i}+1}^{k}-a_{d}}{C}: i=1, \ldots, d_{j} \wedge j_{i}=\ell_{i}-p_{i}, \ldots, \ell_{i}\right) .
$$

Since we only use global dyadic bisection between two consecutive levels, we see that these knots depend only on $d, \widehat{\mathcal{T}}_{0}$ and $\left(p_{1}, \ldots, p_{d}\right)$ but not on the level $k$. This shows $\|\widetilde{\beta}\|_{L^{\infty}\left(\widehat{T}_{\widehat{\beta}}\right)} \lesssim 1$. The hidden multiplicative constant depends only on $d, \widehat{\mathcal{T}}_{0}$ and $\left(p_{1}, \ldots, p_{d}\right)$.

We adopt the approach of Ref. 41 For $\widehat{v} \in L^{2}(\widehat{\Omega})$, we abbreviate $\left\langle\widehat{\beta}^{*}, \widehat{v}\right\rangle:=$ $\int_{\widehat{T}_{\widehat{\beta}}} \widehat{\beta}^{*} \widehat{v} d t$ and define

$$
\widehat{J}_{\bullet}: L^{2}(\widehat{\Omega}) \rightarrow \widehat{\mathcal{X}}_{\bullet}, \quad \widehat{J}_{\bullet} \widehat{v}:=\sum_{k=0}^{M_{\bullet}-1} \sum_{\substack{\left.\widehat{\beta} \in \widehat{\mathcal{H}}_{\bullet} \wedge \widehat{\beta}\right|_{\partial \widehat{\Omega}}=0 \\ \operatorname{level}(\widehat{\beta})=k}}\left\langle\widehat{\beta}^{*}, \widehat{v}\right\rangle \operatorname{Trunc} \bullet(\widehat{\beta})
$$

Before we prove the properties $(\mathrm{S} 4)-(\mathrm{S} 6)$, we collect some basic properties of $\widehat{J}_{\bullet}$.

Lemma 5.6. Let $\widehat{\mathcal{T}}_{\bullet} \in \widehat{\mathbb{T}}$. Then, $\widehat{J}_{\bullet}$ is a projection, i.e.

$$
\widehat{J}_{\bullet} \widehat{V}_{\bullet}=\widehat{V}_{\bullet} \quad \text { for all } \widehat{V}_{\bullet} \in \widehat{\mathcal{X}}_{\bullet} .
$$

Moreover, $\widehat{J}_{\bullet}$ is locally $L^{2}$-stable, i.e. there exists $C_{J}>0$ such that for all $\widehat{T} \in \widehat{\mathcal{T}}_{\bullet}$ :

$$
\|\widehat{J} \bullet \widehat{v}\|_{L^{2}(\widehat{T})} \leq C_{J}\|\widehat{v}\|_{L^{2}\left(\pi_{\bullet}^{2(p+1)}(\widehat{T})\right)} \quad \text { for all } \widehat{v} \in L^{2}(\widehat{\Omega}) .
$$

The constant $C_{J}$ depends only on $d, \widehat{\mathcal{T}}_{0}$, and $\left(p_{1}, \ldots, p_{d}\right)$.

Proof. We prove the lemma in two steps.

Step 1: The projection property 5.22 can be proved as in Theorem 4 of Ref. 41 . There a corresponding projection onto $\widehat{\mathcal{Y}}_{\bullet}$ instead of $\widehat{\mathcal{X}}_{\bullet}=\widehat{\mathcal{Y}}_{\bullet} \cap H_{0}^{1}(\widehat{\Omega})$ is considered. However, with (5.16), the proof works exactly the same. 
Step 2: We prove (5.23). The triangle inequality proves that

$$
\|\widehat{J} \bullet \widehat{v}\|_{L^{2}(\widehat{T})} \leq \sum_{k=0}^{M_{\bullet}-1} \sum_{\substack{\left.\widehat{\beta} \in \mathcal{\mathcal { H }} \bullet \wedge \widehat{\beta}\right|_{\partial \widehat{\Omega}}=0 \\ \operatorname{supp}(\widehat{\beta}) \cap \widehat{T} \mid>0 \\ \operatorname{level}(\widehat{\beta})=k}}\left\|\widehat{\beta}^{*}\right\|_{L^{2}\left(\widehat{T}_{\widehat{\beta}}\right)}\|\widehat{v}\|_{L^{2}\left(\widehat{T}_{\widehat{\beta}}\right)}\|\operatorname{Trunc}(\widehat{\beta})\|_{L^{2}(\widehat{T})} \cdot
$$

By Remark 3.1 it holds that $\operatorname{supp}(\widehat{\beta}) \subseteq \pi_{\bullet}^{2(p+1)}(\widehat{T})$ if $\widehat{\beta} \in \widehat{\mathcal{H}}_{\bullet}$ with $|\operatorname{supp}(\widehat{\beta}) \cap \widehat{T}|>0$. Therefore, we obtain that

$$
\|\widehat{J} \bullet \widehat{v}\|_{L^{2}(\widehat{T})} \leq\|\widehat{v}\|_{L^{2}\left(\pi_{\bullet}^{2(p+1)}(\widehat{T})\right)} \sum_{\substack{\widehat{\beta} \in \widehat{\mathcal{H}} \bullet \\ \operatorname{supp}(\widehat{\beta}) \subseteq \pi_{\bullet}^{2(p+1)}(\widehat{T})}}\left\|\widehat{\beta}^{*}\right\|_{L^{2}\left(\widehat{T}_{\widehat{\beta}}\right)}\|\operatorname{Trunc} \bullet(\widehat{\beta})\|_{L^{2}(\widehat{T})} .
$$

We consider the set $\left\{\widehat{\beta} \in \widehat{\mathcal{H}}_{\bullet}: \operatorname{supp}(\widehat{\beta}) \subseteq \pi_{\bullet}^{2(p+1)}(\widehat{T})\right\}$. Since the support of each basis function in $\widehat{\mathcal{H}}_{\bullet}$ consists of elements in $\widehat{\mathcal{T}}_{\bullet}$, see (3.14), this set is a subset of $\left\{\widehat{\beta} \in \widehat{\mathcal{H}}_{\bullet}: \exists \widehat{T}^{\prime} \in \Pi_{\bullet}^{2(p+1)}(\widehat{T})\right.$ with $\left.\widehat{T}^{\prime} \subseteq \operatorname{supp}(\widehat{\beta})\right\}$. By (M2) and Proposition 3.2 the cardinality of the latter set is bounded by a constant $C>0$ that depends only on $d$ and $\left(p_{1}, \ldots, p_{d}\right)$. With (M1), (5.15) and (5.20), we see that for $\widehat{\beta} \in \widehat{\mathcal{H}}$ • with $\operatorname{supp}(\widehat{\beta}) \subseteq \pi_{\bullet}^{2(p+1)}(\widehat{T})$, it holds that

$$
\left\|\widehat{\beta}^{*}\right\|_{L^{2}\left(\widehat{T}_{\widehat{\beta}}\right)}\|\operatorname{Trunc} \bullet(\widehat{\beta})\|_{L^{2}(\widehat{T})} \leq\left|\widehat{T}_{\widehat{\beta}}\right|^{1 / 2}|\widehat{T}|^{1 / 2}\left\|\widehat{\beta}^{*}\right\|_{L^{\infty}\left(\widehat{T}_{\widehat{\beta}}\right)} \lesssim 1 .
$$

The hidden constant depends only on $d, \widehat{\mathcal{T}}_{0}$, and $\left(p_{1}, \ldots, p_{d}\right)$.

We prove (S4) in the parameter domain. Let $\widehat{T} \in \widehat{\mathcal{T}}_{\bullet}, \widehat{v} \in H_{0}^{1}(\widehat{\Omega})$, and $\widehat{V}_{\bullet} \in \widehat{\mathcal{X}}_{\bullet}$ such that $\left.\widehat{v}\right|_{\pi_{\bullet}^{\text {proj }}(\widehat{T})}=\left.\widehat{V}_{\bullet}\right|_{\pi_{\bullet}^{\text {proj }}(\widehat{T})}$ with $k_{\text {proj }}:=2(p+1)$. Remark 3.1 shows that for $\widehat{\beta} \in \widehat{\mathcal{H}}_{\bullet}$ with $|\operatorname{supp}(\widehat{\beta}) \cap \widehat{T}|>0$, it holds that $\operatorname{supp}(\widehat{\beta}) \subseteq \pi_{\bullet}^{\operatorname{proj}}(\widehat{T})$. With this, (5.15), and the projection property 5.22 of $\widehat{J}_{\bullet}$, we conclude that

$$
\begin{aligned}
& \left.(\widehat{J} \cdot \widehat{v})\right|_{\widehat{T}}=\left.\sum_{k=0}^{M_{\bullet}-1} \sum_{\substack{\left.\widehat{\beta} \in \widehat{\mathcal{H}}_{\bullet} \wedge \widehat{\beta}\right|_{\partial \widehat{\Omega}}=0 \\
\operatorname{level}(\widehat{\beta})=k}}\left\langle\widehat{\beta}^{*}, \widehat{v}\right\rangle \operatorname{Trunc} \bullet(\widehat{\beta})\right|_{\widehat{T}} \\
& \left.\stackrel{5.15}{=} \sum_{k=0}^{M_{\bullet}-1} \sum_{\substack{\left.\widehat{\beta} \in \widehat{\mathcal{H}} \bullet \wedge \widehat{\beta}\right|_{\partial \widehat{\Omega}=0} \\
\operatorname{supp}(\widehat{\beta}) \subseteq \pi^{\operatorname{proj}}(\widehat{T}) \\
\operatorname{level}(\widehat{\beta})=k}}\left\langle\widehat{\beta}^{*}, \widehat{V}_{\bullet}\right\rangle \operatorname{Trunc} \bullet(\widehat{\beta})\right|_{\widehat{T}} \\
& =\left.\left.\left(\widehat{J} \cdot \widehat{V}_{\bullet}\right)\right|_{\widehat{T}} \stackrel{(5.22)}{=} \widehat{V}_{\bullet}\right|_{\widehat{T}}=\left.\widehat{v}\right|_{\widehat{T}} .
\end{aligned}
$$

Next, we prove $(\mathrm{S} 5)$. Let $\widehat{T} \in \widehat{\mathcal{T}}_{\bullet}, \widehat{v} \in H_{0}^{1}(\widehat{\Omega})$, and $\widehat{V}_{\bullet} \in \widehat{\mathcal{X}}_{\bullet}$. By (5.22)-(5.23), it holds that

$$
\begin{aligned}
& \left\|\left(1-\widehat{J}_{\bullet}\right) \widehat{v}\right\|_{L^{2}(\widehat{T})} \stackrel{5.22}{=}\left\|\widehat{v}-\widehat{V}_{\bullet}\right\|_{L^{2}(\widehat{T})}+\left\|\widehat{J} \bullet\left(\widehat{v}-\widehat{V}_{\bullet}\right)\right\|_{L^{2}(\widehat{T})} \\
& \stackrel{5.23}{\lesssim}\left\|\widehat{v}-\widehat{V}_{\bullet}\right\|_{L^{2}\left(\pi_{\bullet}^{2(p+1)}(\widehat{T})\right)} .
\end{aligned}
$$


To proceed, we distinguish between two cases, first, $\pi_{\bullet}^{4(p+1)}(\widehat{T}) \cap \partial \widehat{\Omega}=\emptyset$ and, second, $\pi_{\bullet}^{4(p+1)}(\widehat{T}) \cap \partial \widehat{\Omega} \neq \emptyset$, i.e. if $\widehat{T}$ is far away from the boundary or not. Note that these cases are equivalent to $\left|\pi_{\bullet}^{4(p+1)}(\widehat{T}) \cap \partial \widehat{\Omega}\right|=0$ respectively $\left|\pi_{\bullet}^{4(p+1)}(\widehat{T}) \cap \partial \widehat{\Omega}\right|>0$, since the elements in the parameter domain are rectangular.

In the first case, we proceed as follows: (3.22) especially proves $1 \in \widehat{\mathcal{Y}}_{\bullet}$ with $1=\sum_{\widehat{\beta} \in \widehat{\mathcal{H}} \bullet} c_{\widehat{\beta}} \widehat{\beta}$ on $\widehat{\Omega}$. With Remark 3.1, we see that $\left|\operatorname{supp}(\widehat{\beta}) \cap \pi_{\bullet}^{2(p+1)}(\widehat{T})\right|>0$ implies $\operatorname{supp}(\widehat{\beta}) \subseteq \pi_{\bullet}^{4(p+1)}(\widehat{T})$. Therefore, the restriction satisfies

$$
\begin{aligned}
1=\left.\sum_{\widehat{\beta} \in \widehat{\mathcal{H}} \bullet} c_{\widehat{\beta}} \widehat{\beta}\right|_{\pi_{\bullet}^{2(p+1)}(\widehat{T})}=\sum_{\substack{\widehat{\beta} \in \widehat{\mathcal{H}}_{\bullet} \\
\left|\operatorname{supp}(\widehat{\beta}) \cap \pi_{\bullet}^{(p+1)}(\widehat{T})\right|>0}} c_{\left.\widehat{\beta} \widehat{\beta}\right|_{\pi^{2(p+1)}(\widehat{T})}} c_{\substack{\widehat{\beta} \in \widehat{\mathcal{H}}_{\bullet} \\
\operatorname{supp}(\widehat{\beta}) \subseteq \pi_{\bullet}^{4(p+1)}(\widehat{T})}} c_{\pi_{\bullet}{ }^{2(p+1)}(\widehat{T})} .
\end{aligned}
$$

We define

$$
\begin{array}{r}
\widehat{V}_{\bullet}:=\widehat{v}_{\pi_{\bullet}^{2(p+1)}(\widehat{T})} \sum_{\substack{\widehat{\beta} \in \widehat{\mathcal{H}}_{\bullet} \\
\operatorname{supp}(\widehat{\beta}) \subseteq \pi_{\bullet}^{4(p+1)}(\widehat{T})}} c_{\widehat{\beta}} \widehat{\beta}, \\
\text { where } \quad \widehat{v}_{\pi_{\bullet}^{2(p+1)}(\widehat{T})}:=\left|\pi_{\bullet}^{2(p+1)}(\widehat{T})\right|^{-1} \int_{\pi_{\bullet}^{2(p+1)}(\widehat{T})} \widehat{v} d t .
\end{array}
$$

In the second case, we set $\widehat{V}_{\bullet}:=0$. For the first case, the Poincaré inequality combined with admissibility concludes the proof, whereas we use the Friedrichs inequality combined with admissibility in the second case. In either case, we obtain $\widehat{V}_{\bullet} \in \widehat{\mathcal{X}}_{\bullet}$ and

$$
\begin{aligned}
\|\widehat{v}-\widehat{V} \bullet\|_{L^{2}\left(\pi_{\bullet}^{2(p+1)}(\widehat{T})\right)} & \lesssim \operatorname{diam}\left(\pi_{\bullet}^{4(p+1)}(\widehat{T})\right)\|\nabla \widehat{v}\|_{L^{2}\left(\pi_{\bullet}^{4(p+1)}(\widehat{T})\right)} \\
& \simeq|\widehat{T}|^{1 / d}\|\nabla \widehat{v}\|_{L^{2}\left(\pi_{\bullet}^{4(p+1)}(\widehat{T})\right)} .
\end{aligned}
$$

The hidden constants depend only on $\widehat{\mathcal{T}}_{0},\left(p_{1}, \ldots, p_{d}\right)$, and the shape of the patch $\pi_{\bullet}^{2(p+1)}(\widehat{T})$ respectively the shape of $\pi_{\bullet}^{4(p+1)}(\widehat{T})$ and of $\pi_{\bullet}^{2(p+1)}(\widehat{T}) \cap \partial \widehat{\Omega}$. However, by admissibility, the number of different patch shapes is bounded itself by a constant which again depends only on $d, \widehat{\mathcal{T}}_{0}$ and $\left(p_{1}, \ldots, p_{d}\right)$.

Finally, we prove (S6). Let again $\widehat{T} \in \widehat{\mathcal{T}}_{\bullet}, \widehat{v} \in H_{0}^{1}(\widehat{\Omega})$. For all $\widehat{V}_{\bullet} \in \widehat{\mathcal{X}}_{\bullet}$ that are constant on $\widehat{T}$, the projection property (5.22) implies that

$$
\begin{aligned}
& \left\|\nabla \widehat{J_{\bullet}} \widehat{v}\right\|_{L^{2}(\widehat{T})} \stackrel{\sqrt[5.222]{=}}{=}\left\|\nabla \widehat{J_{\bullet}}\left(\widehat{v}-\widehat{V}_{\bullet}\right)\right\|_{L^{2}(\widehat{T})} \stackrel{\text { (5.10) }}{\lesssim}|\widehat{T}|^{-1 / d}\left\|\widehat{J}_{\bullet}\left(v-\widehat{V}_{\bullet}\right)\right\|_{L^{2}(\widehat{T})}
\end{aligned}
$$

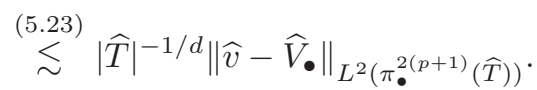

Arguing as before and using (5.24), we conclude the proof. 


\subsection{Verification of (O1)}

The inverse estimate (O1) follows from a standard scaling argument together with the regularity (3.28) of $\gamma$ and the Poincaré-Friedrichs inequality on $\gamma^{-1}(T)$. The constant $C_{\text {inv }}^{\prime}$ depends only on $d, C_{\gamma}, \widehat{\mathcal{T}}_{0}$, and $\left(q_{1}, \ldots, q_{d}\right)$.

\subsection{Verification of $(\mathrm{O} 2)-(\mathrm{O} 4)$}

This section adapts Sec. 3.4 of Ref. 36. Let $W \in \mathcal{P}(\Omega), \mathcal{T}_{\bullet} \in \mathbb{T}$, and $T, T^{\prime} \in \mathcal{T}_{\bullet}$ with $(d-1)$-dimensional intersection $E:=T \cap T^{\prime}$. We set $\widehat{W}:=W \circ \gamma, \widehat{T}:=\gamma^{-1}(T)$, $\widehat{T}^{\prime}:=\gamma^{-1}\left(T^{\prime}\right)$, and $\widehat{E}:=\gamma^{-1}(E)$. Let $\gamma_{\widehat{T}}: \mathbb{R}^{d} \rightarrow \mathbb{R}^{d}$ be the affine transformation with $\widetilde{T}:=\gamma_{\widehat{T}}^{-1}(\widehat{T})=[0,1]^{d}$. Due to admissibility of $\widehat{\mathcal{T}}_{\bullet}$, the number of different configurations for the set $\widetilde{T}^{\prime}:=\gamma_{\widehat{T}}^{-1}\left(\widehat{T}^{\prime}\right)$ is uniformly bounded by a constant that depends only on $d$ and $\widehat{\mathcal{T}}_{0}$. We fix some cut-off function $\widetilde{\varphi} \in H_{0}^{1}\left(\widetilde{T} \cup \widetilde{T}^{\prime}\right)$ with $\widetilde{\varphi}>0$ almost everywhere on $\widetilde{E}:=\gamma_{\widehat{T}}^{-1}(\widehat{E})$. We define $\varphi:=\widetilde{\varphi} \circ \gamma_{\widehat{T}^{\prime}}^{-1} \circ \gamma^{-1}$, and $\widetilde{W}:=W \circ \gamma \circ \gamma_{\widehat{T}}$. Due to the finite dimension of the polynomial space $\widetilde{\mathcal{P}}\left(\widetilde{T} \cup \widetilde{T}^{\prime}\right):=$ $\left\{W^{\prime} \circ \gamma \circ \gamma_{\widehat{T}}: W^{\prime} \in \mathcal{P}(\Omega)\right\}$, there exists $\widetilde{W^{\prime}} \in \widetilde{\mathcal{P}}\left(\widetilde{T} \cup \widetilde{T}^{\prime}\right)$ with $\left.\widetilde{W}\right|_{\widetilde{E}}=\left.\widetilde{W}^{\prime}\right|_{\widetilde{E}}$ and $\left\|\widetilde{W^{\prime} \widetilde{\varphi}}\right\|_{L^{2}\left(\widetilde{T} \cup \widetilde{T}^{\prime}\right)} \lesssim\|\widetilde{W}\|_{L^{2}(\widetilde{E})}$. Finally, we set $W^{\prime}:=\widetilde{W}^{\prime} \circ \gamma_{\widehat{T}^{\prime}}^{-1} \circ \gamma^{-1}$, and $J_{\bullet, E}:=W^{\prime} \varphi$. Standard scaling arguments prove that $(\mathrm{O} 2)-(\mathrm{O} 4)$ are satisfied, where the constants depend only on $d, C_{\gamma}, \widehat{\mathcal{T}}_{0}$, and $\left(q_{1}, \ldots, q_{d}\right)$.

\section{Numerical Examples}

In this section, we apply our adaptive algorithm to the 2D Poisson model problem:

$$
\begin{aligned}
-\Delta u=f & \text { in } \Omega, \\
u=0 & \text { on } \partial \Omega,
\end{aligned}
$$

on different NURBS surfaces $\Omega \subset \mathbb{R}^{2}$. On the parameter domain $\widehat{\Omega}=(0,1)^{d}$, let $p_{1}^{\gamma}, p_{2}^{\gamma} \geq 1$ be fixed polynomial degrees and $\widehat{\mathcal{K}}^{\gamma}$ be an arbitrary fixed two-dimensional vector of $p_{i}^{\gamma}$-open knot vectors with multiplicity smaller or equal to $p_{i}^{\gamma}$ for the interior knots, i.e.

$$
\widehat{\mathcal{K}}^{\gamma}=\left(\widehat{\mathcal{K}}_{1}^{\gamma}, \widehat{\mathcal{K}}_{2}^{\gamma}\right)
$$

see also Sec. 3.1. Let $\widehat{\mathcal{B}}^{\gamma}$ be the corresponding tensor-product B-spline basis, i.e.

$$
\widehat{\mathcal{B}}^{\gamma}=\left\{\widehat{B}_{j_{1}, j_{2}}^{\gamma}: j_{i} \in\left\{0, \ldots, N_{i}^{\gamma}-1\right\}\right\},
$$

where $\widehat{B}_{j_{1}, j_{2}}^{\gamma}$ is defined as in (3.3). For given points in the plane $c_{j_{1}, j_{2}}^{\gamma} \in \mathbb{R}^{2}$ and positive weights $w_{j_{1}, j_{2}}^{\gamma}>0$ for $j_{i} \in\left\{0, \ldots, N_{i}^{\gamma}-1\right\}$, a NURBS-surface $\Omega$ is defined via a parametrization $\gamma: \widehat{\Omega} \rightarrow \Omega$ of the form

$$
\gamma\left(s_{1}, s_{2}\right)=\frac{\sum_{\substack{i \in\{1,2\} \\ j_{i} \in\left\{0, \ldots, N_{i}^{\gamma}-1\right\}}} w_{j_{1}, j_{2}}^{\gamma} c_{j_{1}, j_{2}}^{\gamma} \widehat{B}_{j_{1}, j_{2}}^{\gamma}\left(s_{1}, s_{2}\right)}{\sum_{\substack{i \in\{1,2\} \\ j_{i} \in\left\{0, \ldots, N_{i}^{\gamma}-1\right\}}} w_{j_{1}, j_{2}}^{\gamma} \widehat{B}_{j_{1}, j_{2}}^{\gamma}\left(s_{1}, s_{2}\right)} .
$$




\subsection{Square}

In the first experiment, we consider the unit square $\Omega=(0,1)^{2}$, where we choose $p_{1}^{\gamma}, p_{2}^{\gamma}=1$ and $\widehat{\mathcal{K}}_{1}^{\gamma}=\widehat{\mathcal{K}}_{2}^{\gamma}=(0,0,1,1)$. We set the control points:

$$
c_{1,1}^{\gamma}=(0,0), \quad c_{2,1}^{\gamma}=(1,0), \quad c_{1,2}^{\gamma}=(0,1), \quad c_{2,2}^{\gamma}=(1,1)
$$

and all weights equal to 1 . We choose $f$ as in Example 7.4 of Ref. 9] such that the exact solution of (6.1) is given by $u\left(x_{1}, x_{2}\right)=x_{1}^{2.3}\left(1-x_{1}\right) x_{2}^{2.9}\left(1-x_{2}\right)$, which implies $u \in H^{2}(\Omega) \backslash H^{3}(\Omega)$. To create the initial ansatz space with spline degrees $p_{1}=p_{2} \in\{2,3,4\}$, we choose the initial knot vectors $\widehat{\mathcal{K}}_{1}^{0}=\widehat{\mathcal{K}}_{2}^{0}=(0, \ldots, 0,1, \ldots, 1)$, where the multiplicity of 0 and 1 is $p_{1}+1=p_{2}+1$. We apply Algorithm 2.1 for the spline degrees 2,3 and 4 with uniform $(\theta=1)$ and adaptive $(\theta=0.5)$ meshrefinement. Some adaptively generated hierarchical meshes are shown in Fig. 3. In Fig. 4 we plot the energy error $\left\|\nabla u-\nabla U_{\ell}\right\|_{L^{2}(\Omega)}$ as well as the error estimator $\eta_{\ell}$ against the number of elements $\# \mathcal{T}_{\ell}$. Due to the regularity of $u$, we obtain a suboptimal convergence rate for $p_{1}=p_{2}>2$. The adaptive strategy on the

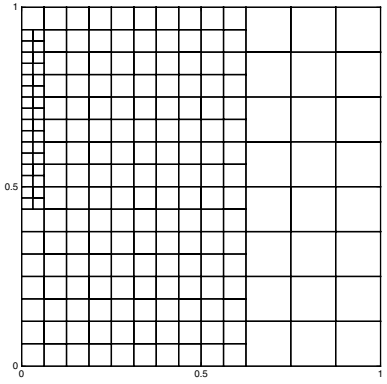

$\# \mathcal{T}_{6}=208$

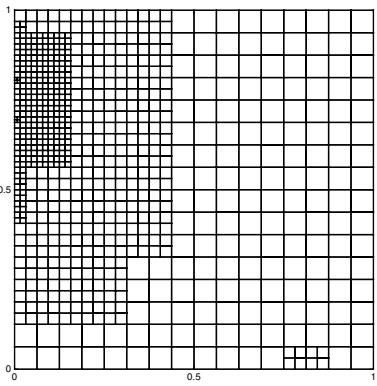

$\# \mathcal{T}_{8}=742$

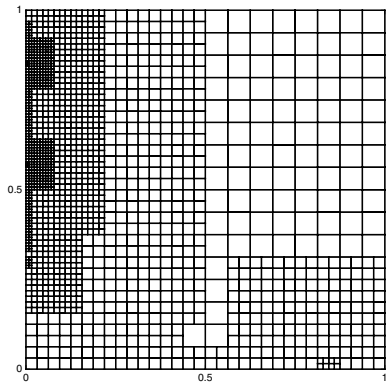

$\# \mathcal{T}_{10}=1633$

Fig. 3. Hierarchical meshes generated by Algorithm 2.1 with $\theta=0.5$ for the problem of Sec.6.1 for $p_{1}=p_{2}=4$.
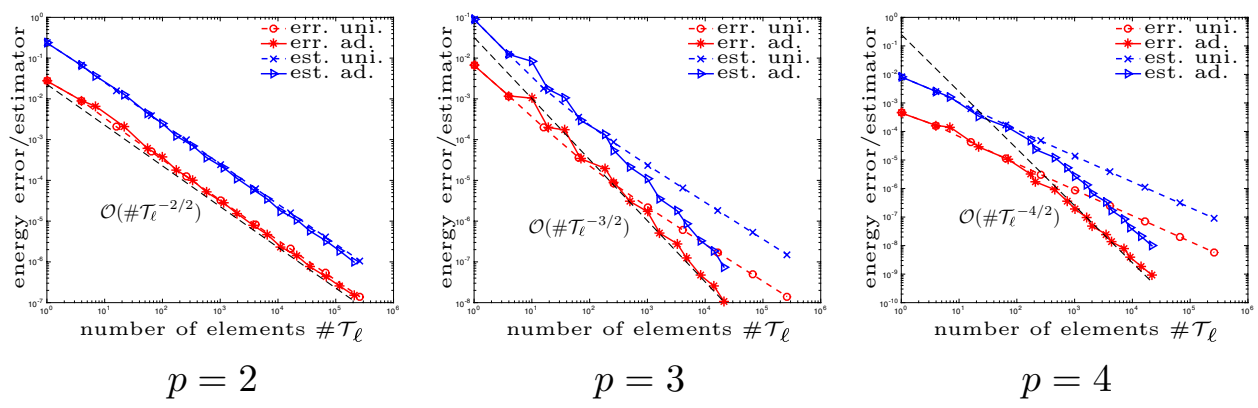

Fig. 4. Energy error $\left\|\nabla u-\nabla U_{\ell}\right\|_{L^{2}(\Omega)}$ and error estimator $\eta_{\ell}$ for the problem of Sec. 6.1 on the unit square for uniform $(\theta=1)$ and adaptive $(\theta=0.5)$ mesh-refinement and different spline orders $p_{1}=p_{2}=p \in\{2,3,4\}$, where adaptivity always regains the respective optimal convergence rate. 
other hand recovers the optimal convergence of the energy error and the error estimator, i.e. $\mathcal{O}\left(\left(\# \mathcal{T}_{\ell}\right)^{-p / 2}\right)$. However, here and also in the following examples, one observes an enlarged preasymptotic range as $p$ increases. Indeed, in order to preserve admissibility of the meshes, the adaptive algorithm has to refine not only the marked elements but also some additional elements depending on the supports of the basis functions. Since the supports become larger for higher polynomial degrees $p$, the number of these additional elements increases. In particular, this may lead to nearly uniform refinement at the beginning and thus to a worse preasymptotic behavior.

\subsection{L-shape}

To obtain the L-shaped domain $\Omega=(0,1)^{2} \backslash([0,0.5] \times[0,0.5])$, we choose $p_{1}^{\gamma}, p_{2}^{\gamma}=1$ and $\widehat{\mathcal{K}}_{1}^{\gamma}=(0,0,0.5,1,1), \widehat{\mathcal{K}}_{2}^{\gamma}=(0,0,1,1)$. Moreover, we choose the control points:

$$
\begin{aligned}
& c_{1,1}^{\gamma}=(0,0.5), \quad c_{2,1}^{\gamma}=(0.5,0.5), \quad c_{3,1}^{\gamma}=(0.5,0), \\
& c_{1,2}^{\gamma}=(0,1), \quad c_{2,2}^{\gamma}=(1,1), \quad c_{3,2}^{\gamma}=(1,0),
\end{aligned}
$$

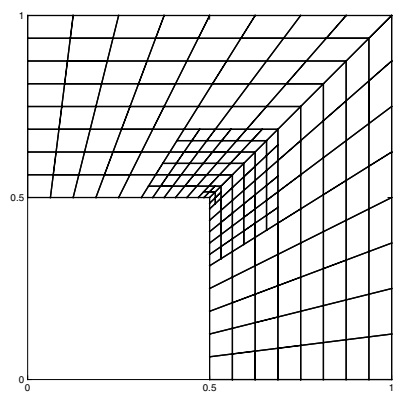

$\# \mathcal{T}_{6}=188$

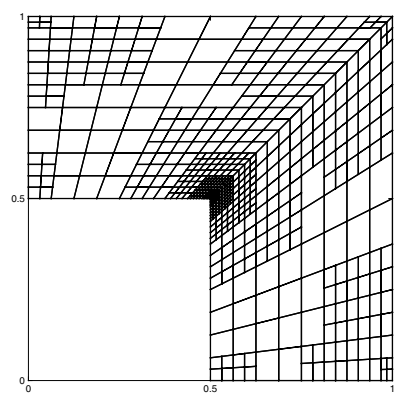

$\# \mathcal{T}_{9}=632$

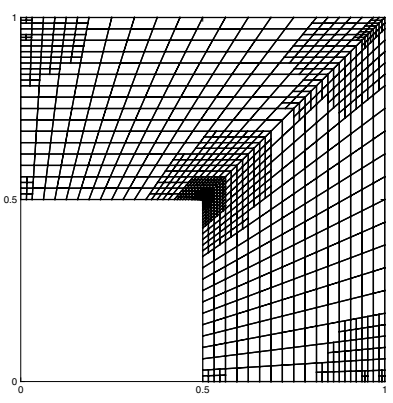

$\# \mathcal{T}_{11}=1364$

Fig. 5. Hierarchical meshes generated by Algorithm 2.1 with $\theta=0.4$ for the problem of Sec. 6.2 for $p_{1}=p_{2}=2$.
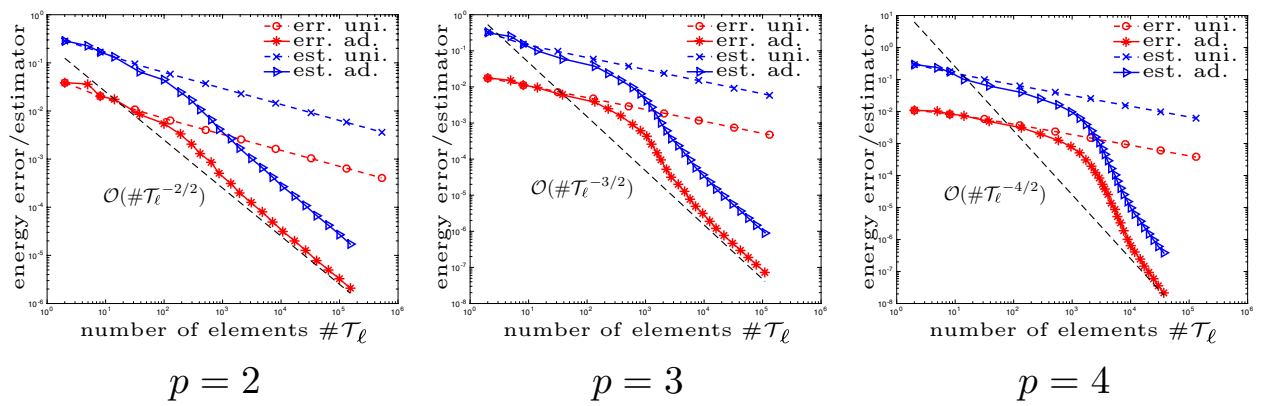

Fig. 6. Energy error $\left\|\nabla u-\nabla U_{\ell}\right\|_{L^{2}(\Omega)}$ and error estimator $\eta_{\ell}$ for the problem of Sec. 6.3 on the L-shape for uniform $(\theta=1)$ and adaptive $(\theta=0.5)$ mesh-refinement and different spline orders $p_{1}=p_{2}=p \in\{2,3,4\}$, where adaptivity always regains the respective optimal convergence rate. 
and set all weights equal to 1 . We consider the Poisson problem (6.1) with $f=1$. For the initial ansatz space with spline degrees $p_{1}=p_{2} \in\{2,3,4\}$, we choose the initial knot vectors $\widehat{\mathcal{K}}_{1}^{0}=(0, \ldots, 0,0.5, \ldots, 0.5,1, \ldots, 1)$ and $\widehat{\mathcal{K}}_{2}^{0}=(0, \ldots, 0,1 \ldots, 1)$, where the multiplicity of 0 and 1 is $p_{1}+1=p_{2}+1$, whereas the multiplicity of 0.5 is $p_{1}$. As a consequence, the ansatz functions are only continuous at $\{0.5\} \times[0,1]$, but not continuously differentiable. We compare uniform $(\theta=1)$ and adaptive $(\theta=0.4)$ mesh-refinement. In Fig. 5, one can see some adaptively generated hierarchical meshes. In Fig. 6, we plot again the energy error $\left\|\nabla u-\nabla U_{\ell}\right\|_{L^{2}(\Omega)}$ and the error estimator $\eta_{\ell}$ against the number of elements $\# \mathcal{T}_{\ell}$. The uniform approach leads to a suboptimal convergence rate, since the re-entrant corner at $(0.5,0.5)$ causes a generic singularity of the solution $u$. However, the adaptive strategy recovers the optimal convergence rate $\mathcal{O}\left(\left(\# \mathcal{T}_{\ell}\right)^{-p / 2}\right)$. Again, one observes a worse preasymptotic behavior for higher polynomial degrees $p$.

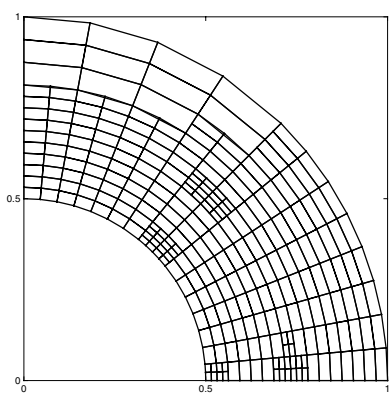

$\# \mathcal{T}_{4}=265$

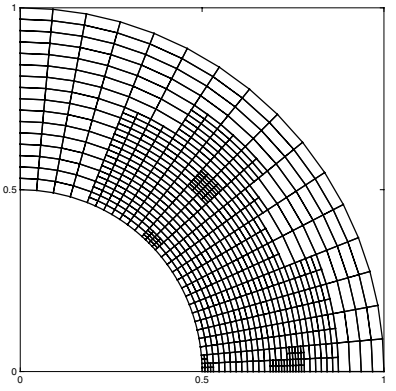

$\# \mathcal{T}_{5}=718$

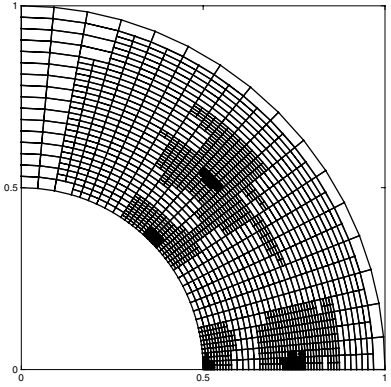

$\# \mathcal{T}_{6}=1777$

Fig. 7. Hierarchical meshes generated by Algorithm 2.1 with $\theta=0.8$ for the problem of Sec.6.3 for $p_{1}=p_{2}=3$.
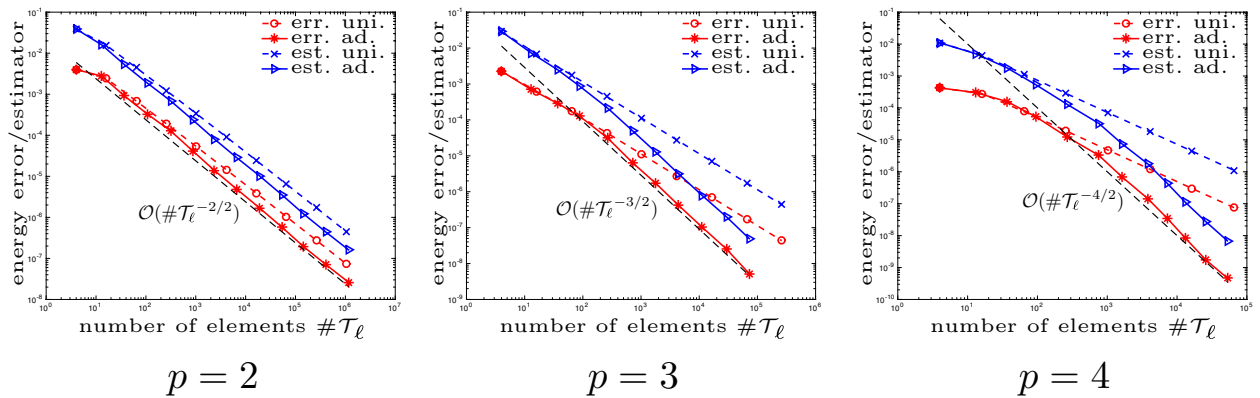

Fig. 8. Energy error $\left\|\nabla u-\nabla U_{\ell}\right\|_{L^{2}(\Omega)}$ and error estimator $\eta_{\ell}$ for the problem of Sec. 6.3 on the quarter ring for uniform $(\theta=1)$ and adaptive $(\theta=0.8)$ mesh-refinement and different spline orders $p_{1}=p_{2}=p \in\{2,3,4\}$, where adaptivity always regains the respective optimal convergence rate. 


\subsection{Quarter ring}

We construct the NURBS-surface given in polar coordinates $\Omega=\{r(\cos (\varphi, \sin (\varphi)) \mid$ $0.5<r<1 \wedge 0<\varphi<\pi / 2\}$ by choosing $p_{1}^{\gamma}=2, p_{2}^{\gamma}=1$ and $\widehat{\mathcal{K}}_{1}^{\gamma}=(0,0,0$, $1,1,1), \widehat{\mathcal{K}}_{2}^{\gamma}=(0,0,1,1)$. Moreover, we choose the control points:

$$
\begin{aligned}
& c_{1,1}^{\gamma}=(0,0.5), \quad c_{2,1}^{\gamma}=(0.5,0.5), \quad c_{3,1}^{\gamma}=(0.5,0), \\
& c_{1,2}^{\gamma}=(0,1), \quad c_{2,2}^{\gamma}=(1,1), \quad c_{3,2}^{\gamma}=(1,0),
\end{aligned}
$$

and set all weights, except $w_{2,1}^{\gamma}, w_{2,2}^{\gamma}:=1 / \sqrt{2}$, equal to 1 . As right-hand side in (6.1), we choose the indicator function $f=\chi_{S}$, where $S=\{r(\cos (\varphi), \sin (\varphi)) \mid 0.5<$ $r<0.75 \wedge 0<\varphi<\pi / 4\}=\gamma((0.5,1) \times(0.5,1))$. For the initial ansatz space with spline degrees $p_{1}=p_{2} \in\{2,3,4\}$, we choose the initial knot vectors $\widehat{\mathcal{K}}_{1}^{0}=\widehat{\mathcal{K}}_{2}^{0}=$ $(0, \ldots, 0,0.5, \ldots, 0.5,1 \ldots, 1)$, where the multiplicity of 0 and 1 is $p_{1}+1=p_{2}+1$, whereas the multiplicity of 0.5 is $p_{1}=p_{2}$. As a consequence the ansatz functions are only continuous at $\{0.5\} \times[0,1]$ and $[0,1] \times\{0.5\}$. We compare uniform $(\theta=1)$ and adaptive $(\theta=0.8)$ mesh-refinement. Some adaptively generated hierarchical meshes are shown in Fig. 7. In Fig. 8 we plot the energy error $\left\|\nabla u-\nabla U_{\ell}\right\|_{L^{2}(\Omega)}$ and the error estimator $\eta_{\ell}$ against the number of elements $\# \mathcal{T}_{\ell}$. For $p_{1}=p_{2}>2$, uniformrefinement leads to suboptimal convergence rate. However, the adaptive approach regains the optimal rate $\mathcal{O}\left(\left(\# \mathcal{T}_{\ell}\right)^{-p / 2}\right)$. Again, one observes a worse preasymptotic behavior for higher polynomial degrees $p$.

\section{Acknowledgment}

The authors acknowledge support through the Austrian Science Fund (FWF) under Grant P29096, Grant W1245, Grant P27005 and Grant SFB F65.

\section{References}

1. M. Ainsworth and J. T. Oden, A posteriori Error Estimation in Finite Element Analysis, Pure and Applied Mathematics (John Wiley \& Sons, 2000).

2. M. Aurada, S. Ferraz-Leite and D. Praetorius, Estimator reduction and convergence of adaptive BEM, Appl. Numer. Math. 62 (2012) 787-801.

3. Y. Bazilevs, L. Beirão da Veiga, J. A. Cottrell, T. J. R. Hughes and G. Sangalli, Isogeometric analysis: Approximation, stability and error estimates for h-refined meshes, Math. Models Methods Appl. Sci. 16 (2006) 1031-1090.

4. L. Beirão da Veiga, F. Brezzi, A. Cangiani, G. Manzini, L. Donatella Marini and A. Russo, Basic principles of virtual element methods, Math. Models Methods Appl. Sci. 23 (2013) 199-214.

5. L. Beirão da Veiga, A. Buffa, G. Sangalli and R. Vázquez, Analysis-suitable T-splines of arbitrary degree: Definition, linear independence and approximation properties, Math. Models Methods Appl. Sci. 23 (2013) 1979-2003.

6. L. Beirão da Veiga, A. Buffa, G. Sangalli and R. Vázquez, Mathematical analysis of variational isogeometric methods, Acta Numerica 23 (2014) 157-287. 
7. A. Bespalov, A. Haberl and D. Praetorius, Adaptive FEM with coarse initial mesh guarantees optimal convergence rates for compactly perturbed elliptic problems, Comput. Methods Appl. Mech. Eng. 317 (2017) 318-340.

8. P. Binev, W. Dahmen and R. DeVore, Adaptive finite element methods with convergence rates, Numer. Math. 97 (2004) 219-268.

9. A. Buffa and E. M. Garau, A posteriori error estimators for hierarchical B-spline discretizations, preprint (2016), arXiv:1611.07816.

10. A. Buffa and E. M. Garau, Refinable spaces and local approximation estimates for hierarchical splines, IMA J. Numer. Anal. 37 (2016) 1125-1149.

11. A. Buffa and C. Giannelli, Adaptive isogeometric methods with hierarchical splines: Error estimator and convergence, Math. Models Methods Appl. Sci. 26 (2016) 1-25.

12. A. Buffa and C. Giannelli, Adaptive Isogeometric Methods with Hierarchical Splines: Optimality and Convergence Rates, Technical Report (École Polytechnique Fédérale de Lausanne, March, 2017).

13. A. Buffa, C. Giannelli, P. Mørgenstern and D. Peterseim, Complexity of hierarchical refinement for a class of admissible mesh configurations, Comput.-Aided Geom. Design 47 (2016) 83-92.

14. C. Carstensen, M. Feischl, M. Page and D. Praetorius, Axioms of adaptivity, Comput. Math. Appl. 67 (2014) 1195-1253.

15. J. M. Cascon, C. Kreuzer, R. H. Nochetto and K. G. Siebert, Quasi-optimal convergence rate for an adaptive finite element method, SIAM J. Numer. Anal. 46 (2008) $2524-2550$.

16. J. A. Cottrell, T. J. R. Hughes and Y. Bazilevs, Isogeometric Analysis: Toward Integration of CAD and FEA (John Wiley \& Sons, 2009).

17. C. De Boor, A Practical Guide to Splines, rev. edn. (Springer, 2001).

18. T. Dokken, T. Lyche and K. F. Pettersen, Polynomial splines over locally refined box-partitions, Comput.-Aided Geom. Design 30 (2013) 331-356.

19. W. Dörfler, A convergent adaptive algorithm for Poisson's equation, SIAM J. Numer. Anal. 33 (1996) 1106-1124.

20. C. Erath, Adaptive Finite Volumen Methode, Master's Thesis (Institute for Analysis and Scientific Computing, TU Wien, 2005).

21. M. Feischl, T. Führer and D. Praetorius, Adaptive FEM with optimal convergence rates for a certain class of nonsymmetric and possibly nonlinear problems, SIAM J. Numer. Anal. 52 (2014) 601-625.

22. M. Feischl, G. Gantner, A. Haberl and D. Praetorius, Adaptive 2D IGA boundary element methods, Eng. Anal. Bound. Element 62 (2016) 141-153.

23. M. Feischl, G. Gantner, A. Haberl and D. Praetorius, Optimal convergence for adaptive IGA boundary element methods for weakly-singular integral equations, Numer. Math. 136 (2017) 147-182.

24. M. Feischl, G. Gantner and D. Praetorius, Reliable and efficient a posteriori error estimation for adaptive IGA boundary element methods for weakly-singular integral equations, Comput. Methods Appl. Mech. Eng. 290 (2015) 362-386.

25. F. D. Gaspoz and P. Morin, Convergence rates for adaptive finite elements, IMA J. Numer. Anal. 29 (2008) 917-936.

26. C. Giannelli, B. Jüttler and H. Speleers, THB-splines: The truncated basis for hierarchical splines, Comput.-Aided Geom. Design 29 (2012) 485-498.

27. C. Giannelli, B. Jüttler and H. Speleers, Strongly stable bases for adaptively refined multilevel spline spaces, Adv. Comput. Math. 40 (2014) 459-490. 
28. P. Hennig, M. Kästner, P. Mørgenstern and D. Peterseim, Adaptive mesh refinement strategies in isogeometric analysis - A computational comparison, Comput. Methods Appl. Mech. Eng. 316 (2017) 424-448.

29. T. J. R. Hughes, J. A. Cottrell and Y. Bazilevs, Isogeometric analysis: CAD, finite elements, NURBS, exact geometry and mesh refinement, Comput. Methods Appl. Mech. Eng. 194 (2005) 4135-4195.

30. K. A. Johannessen, T. Kvamsdal and T. Dokken, Isogeometric analysis using LR B-splines, Comput. Methods Appl. Mech. Eng. 269 (2014) 471-514.

31. K. A. Johannessen, F. Remonato and T. Kvamsdal, On the similarities and differences between classical hierarchical, truncated hierarchical and LR B-splines, Comput. Methods Appl. Mech. Eng. 291 (2015) 64-101.

32. G. Kuru, C. V. Verhoosel, K. G. Van der Zee and E. Harald van Brummelen, Goal-adaptive isogeometric analysis with hierarchical splines, Comput. Methods Appl. Mech. Eng. 270 (2014) 270-292.

33. P. Mørgenstern, Globally structured three-dimensional analysis-suitable T-splines: Definition, linear independence and $m$-graded local refinement, SIAM J. Numer. Anal. 54 (2016) 2163-2186.

34. P. Mørgenstern and D. Peterseim, Analysis-suitable adaptive T-mesh refinement with linear complexity, Comput.-Aided Geom. Design 34 (2015) 50-66.

35. P. Morin, R. H. Nochetto and K. G. Siebert, Data oscillation and convergence of adaptive FEM, SIAM J. Numer. Anal. 38 (2000) 466-488.

36. R. H. Nochetto and A. Veeser, Primer of adaptive finite element methods, in Multiscale and Adaptivity: Modeling, Numerics and Applications, Lecture Notes in Mathematics, Vol. 2040 (Springer 2012), pp. 125-225.

37. W. Rudin, Functional Analysis, 2nd edn. (McGraw-Hill, 1991).

38. S. Schimanko, Adaptive Isogeometric Boundary Element Method for the HyperSingular Integral Equation, Master's Thesis (Institute for Analysis and Scientific Computing, TU Wien, 2016).

39. L. Schumaker, Spline Functions: Basic Theory (Cambridge Univ. Press, 2007).

40. M. A. Scott, X. Li, T. W. Sederberg and T. J. R. Hughes, Local refinement of analysissuitable T-splines, Comput. Methods Appl. Mech. Eng. 213 (2012) 206-222.

41. H. Speleers and C. Manni, Effortless quasi-interpolation in hierarchical spaces, Numer. Math. 132 (2016) 155-184.

42. R. Stevenson, Optimality of a standard adaptive finite element method, Found. Comput. Math. 7 (2007) 245-269.

43. R. Stevenson, The completion of locally refined simplicial partitions created by bisection, Math. Comput. 77 (2008) 227-241.

44. R. Verfürth, A posteriori Error Estimation Techniques for Finite Element Methods (Oxford Univ. Press, 2013).

45. A.-V. Vuong, C. Giannelli, B. Jüttler and B. Simeon, A hierarchical approach to adaptive local refinement in isogeometric analysis, Comput. Methods Appl. Mech. Eng. 200 (2011) 3554-3567. 